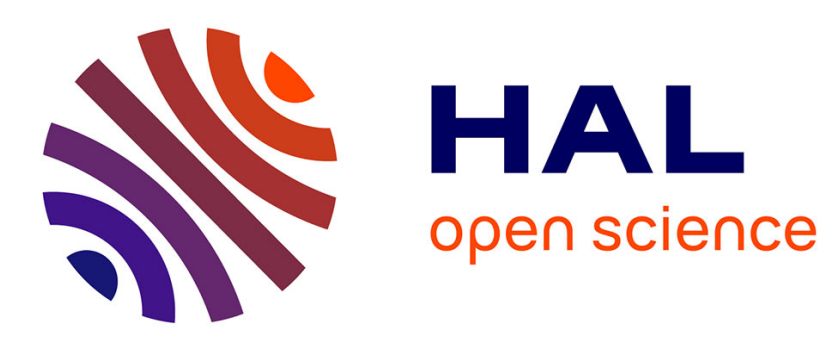

\title{
Random vibro-acoustic control of internal noise through optimized Tuned Mass Dampers
}

Elyes Mrabet, Mohamed Ichchou, Noureddine Bouhaddi

\section{To cite this version:}

Elyes Mrabet, Mohamed Ichchou, Noureddine Bouhaddi. Random vibro-acoustic control of internal noise through optimized Tuned Mass Dampers. Mechanical Systems and Signal Processing, 2019, 130, pp.17-40. 10.1016/j.ymssp.2019.04.062 . hal-02300033

\section{HAL Id: hal-02300033 \\ https://hal.science/hal-02300033}

Submitted on 29 Sep 2019

HAL is a multi-disciplinary open access archive for the deposit and dissemination of scientific research documents, whether they are published or not. The documents may come from teaching and research institutions in France or abroad, or from public or private research centers.
L'archive ouverte pluridisciplinaire HAL, est destinée au dépôt et à la diffusion de documents scientifiques de niveau recherche, publiés ou non, émanant des établissements d'enseignement et de recherche français ou étrangers, des laboratoires publics ou privés.

\section{(c)(1)}

Distributed under a Creative Commons Attribution| 4.0 International License 
Systems and Signal Processing

Elsevier Editorial System(tm) for Mechanical Manuscript Draft

Manuscript Number: MSSP18-387R1

Title: Random vibro-acoustic control of internal noise through optimized Tuned Mass Dampers

Article Type: Full Length Article

Keywords: Interior noise control; stochastic acoustic optimization; root mean square acoustic pressure; multiple tuned mass damper; multimodal control of interior sound.

Corresponding Author: Dr. Elyes Mrabet,

Corresponding Author's Institution:

First Author: Elyes Mrabet

Order of Authors: Elyes Mrabet; Mohamed N Ichchou, Professor; Noureddine Bouhaddi, Professor 


\section{Cover letter}

February 27, 2018

Editorial Department of Mechanical Systems and Signal Processing

Dear Editor of MSSP,

I am submitting a manuscript for consideration of publication in Mechanical Systems and Signal Processing. The manuscript is entitled "Random vibro-acoustic control of internal noise through optimized Tuned Mass Dampers".

It has not been published elsewhere and that it has not been submitted simultaneously for publication elsewhere.

The work deals with the passive control of interior sound pressure induced by randomly vibrating structure coupled to an enclosure filled with air. The control in the low frequency range is achieved using a Tuned Mass Damper (TMD) device. In order to guarantee an optimal performance of the TMD device, a stochastic acoustic optimization strategy is proposed. The effectiveness of the proposed strategy is investigated and compared with others from the open literature.

The capability of the proposed optimization strategy to deal with multimodal control of interior sound has also been addressed. To this end, multiple TMDs have been used. The optimal TMD parameters have been obtained using a multi-objective optimization approach and the results that have been gathered show significant attenuations in all target resonant modes.

Thank you very much for your consideration.

Yours Sincerely,

Dr. Elyes Mrabet

Laboratoire de Mécanique, Modélisation et Productique, Ecole Nationale d'Ingénieurs de Sfax, Route Soukra

Km 3.5 B.P 1173-3038, Sfax, Tunisie

elyes.mrabet@isetkr.rnu.tn

Co-author: Prof. Mohamed Najib Ichchou,

LTDS UMR5513 Ecole Centrale de Lyon, Université de Lyon, Ecully, France

Co-author: Prof. Noureddine Bouhaddi

Univ. Bourgogne Franche-Comté, FEMTO-ST Institute, CNRS/UFC/ENSMM/UTBM, Department of Applied Mechanics, 25000 BESANÇON-FR 


\title{
« Random vibro-acoustic control of internal noise through optimized Tuned Mass Dampers»
}

\author{
E. Mrabet, M. N. Ichchou, N. Bouhaddi
}

Submitted for publication in Mechanical Systems and Signal Processing

\section{$\underline{\text { Responses to Reviewer's comments }}$}

NB: All modifications, in the revised version of the manuscript, are printed in red.

\section{Reviewer \# 2}

The authors would like to thank the Reviewer for the interest he showed to their work.

\section{Reviewer \# 4}

The authors would like to thank the Reviewer for his valuable work.

\begin{tabular}{|c|c|c|}
\hline & Comments & Responses \\
\hline 1 & $\begin{array}{l}\text { a) It is not clear to the } \\
\text { reviewer why a stochastic } \\
\text { analysis is used; the force } \\
\text { is limited to a point load is } \\
\text { I guess the same } \\
\text { conclusions of the study } \\
\text { may have been obtained } \\
\text { using a deterministic force. } \\
\text { Isn't it? If not please } \\
\text { explain. } \\
\text { b) It would have been more } \\
\text { illuminating if a distributed } \\
\text { random force such as a } \\
\text { Rain on the roof, a Diffuse } \\
\text { acoustic field or a } \\
\text { Turbulent boundary layer } \\
\text { was used. Please } \\
\text { comment. }\end{array}$ & $\begin{array}{l}\text { a) Generally speaking, when dealing with non deterministic } \\
\text { vibrating systems, a non deterministic (stochastic, for instance) } \\
\text { analysis should be made to evaluate some quantities of interest. In } \\
\text { Ref [42], chapter 10, the authors wrote "Real engineering systems } \\
\text { include some levels of uncertainty that cannot be captured by the } \\
\text { available deterministic analysis methods" . } \\
\text { In our case, the excitation is stochastic (non deterministic excitation), } \\
\text { therefore the system responses will be characterised in statistical } \\
\text { way, the root mean square response, for instance. } \\
\text { Besides, the proposed optimization strategy (based on a stochastic } \\
\text { analysis) has been compared to other optimization strategies based } \\
\text { on deterministic analysis (Den Hartog, APE) and the results were } \\
\text { different. } \\
\text { b) In the presented work, the focus has basically been on the ability } \\
\text { of the TMD devices to efficiently control the coupled modes that } \\
\text { are either dominated by structure mode or by cavity mode. } \\
\text { Since the present manuscript is relatively huge, it can be extended to }\end{array}$ \\
\hline
\end{tabular}




\begin{tabular}{|c|c|c|}
\hline & & ith other \\
\hline 2 & $\begin{array}{l}\text { I understand the argument of } \\
\text { the authors that a control at a } \\
\text { single location is the cavity is } \\
\text { used to mimic a driver's ear } \\
\text { in an automobile... However, } \\
\text { the studied problem is } \underline{\text { far }} \\
\text { from the one of an } \\
\underline{\text { automobile (different }} \\
\text { excitations, geometry, } \\
\text { damping: absorbing materials } \\
\text { are usually used and must be } \\
\text { accounted for even if the } \\
\text { firewall is the targeted } \\
\text { panel...). I suggest that the } \\
\underline{\text { authors at least monitor }} \\
\text { global indicators such as the } \\
\underline{\text { space averaged quadratic }} \\
\underline{\text { velocity (plate) and pressure }} \\
\text { (cavity). Even for the } \\
\text { automobile problem, not sure } \\
\text { the pressure filed is } \\
\text { controlled, say at the } \\
\text { passenger's ear! An } \\
\text { alternative will be to show an } \\
\text { example wherein the control } \\
\text { targets two or more locations } \\
\text { in the cavity. }\end{array}$ & 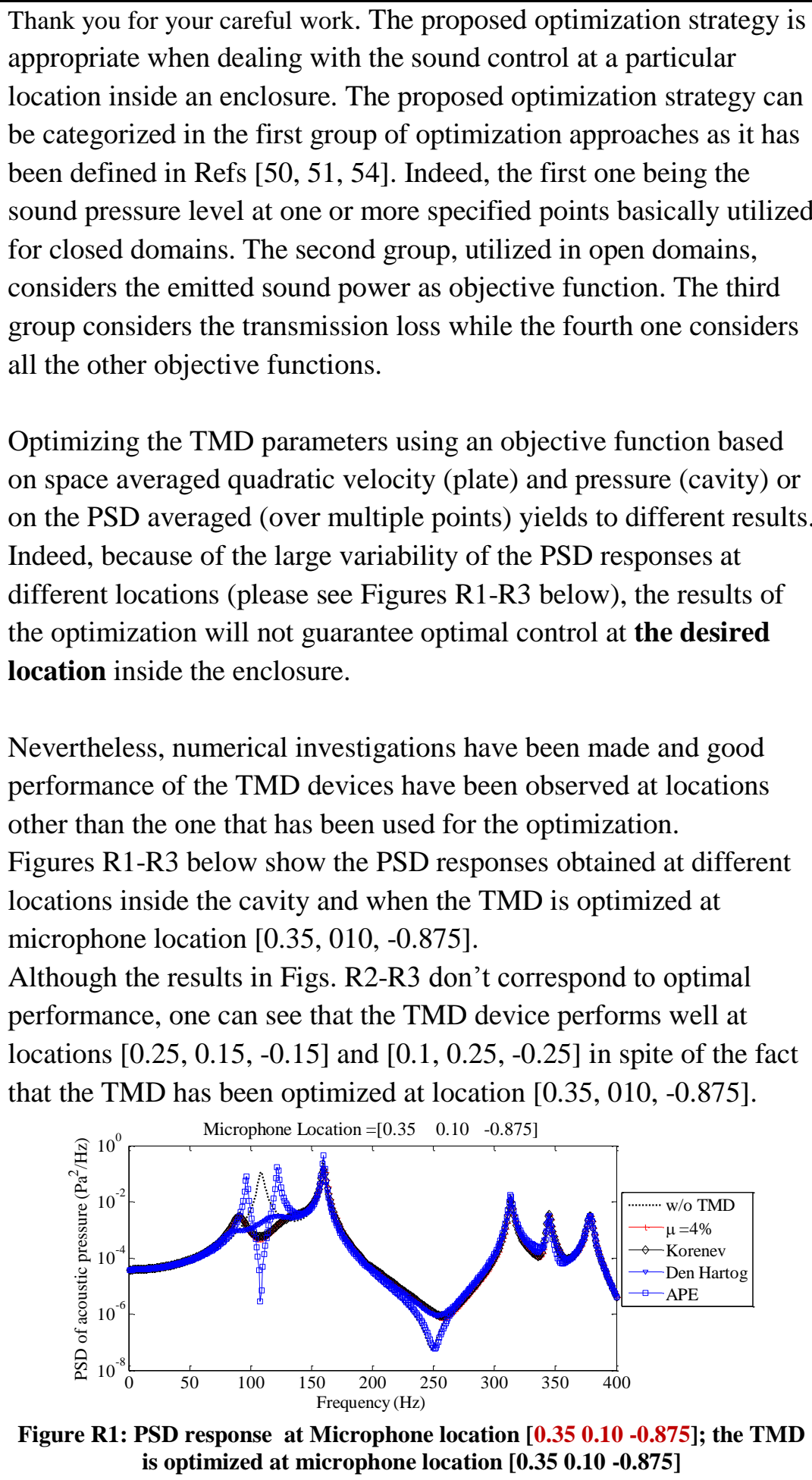 \\
\hline
\end{tabular}




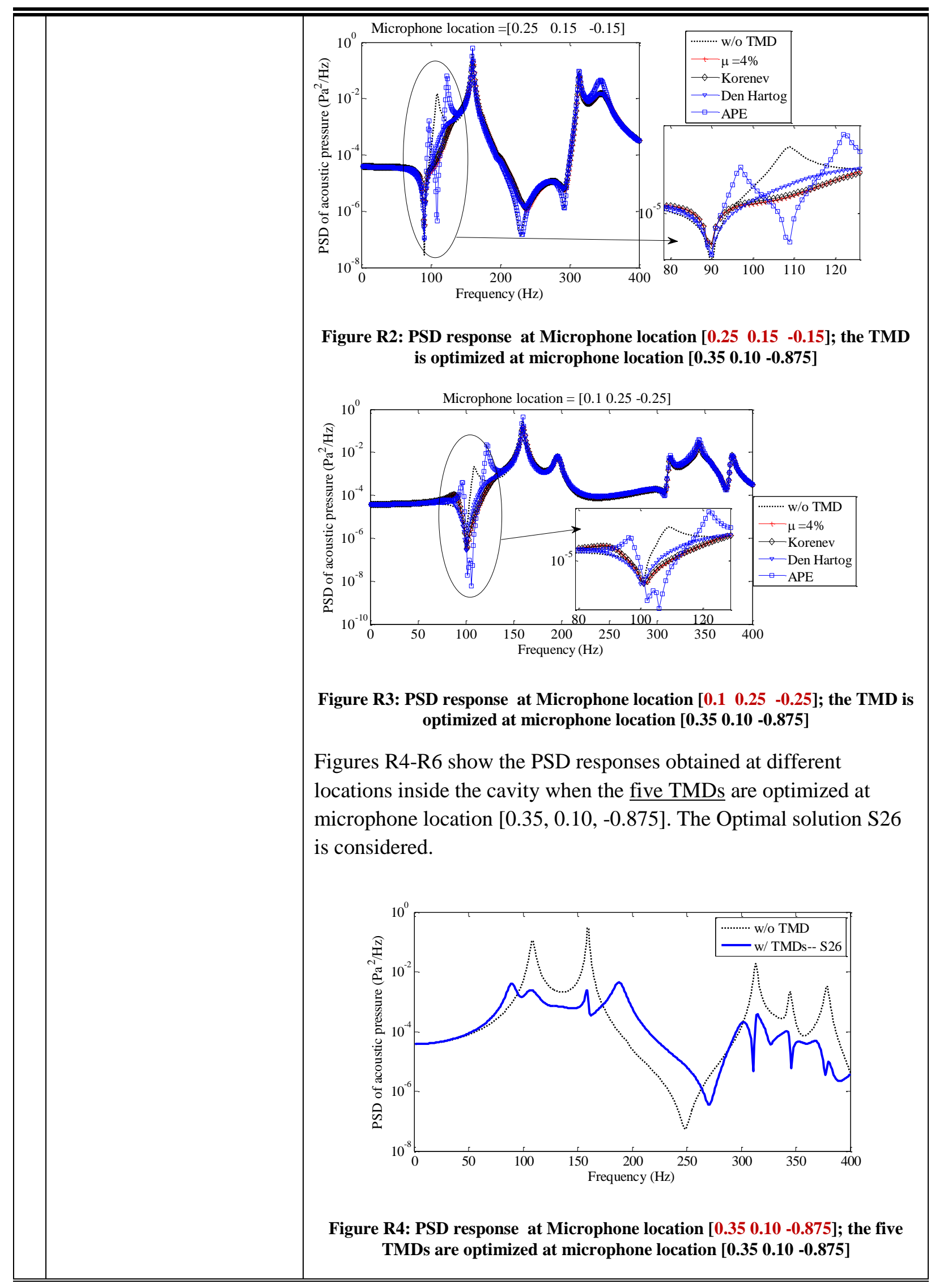




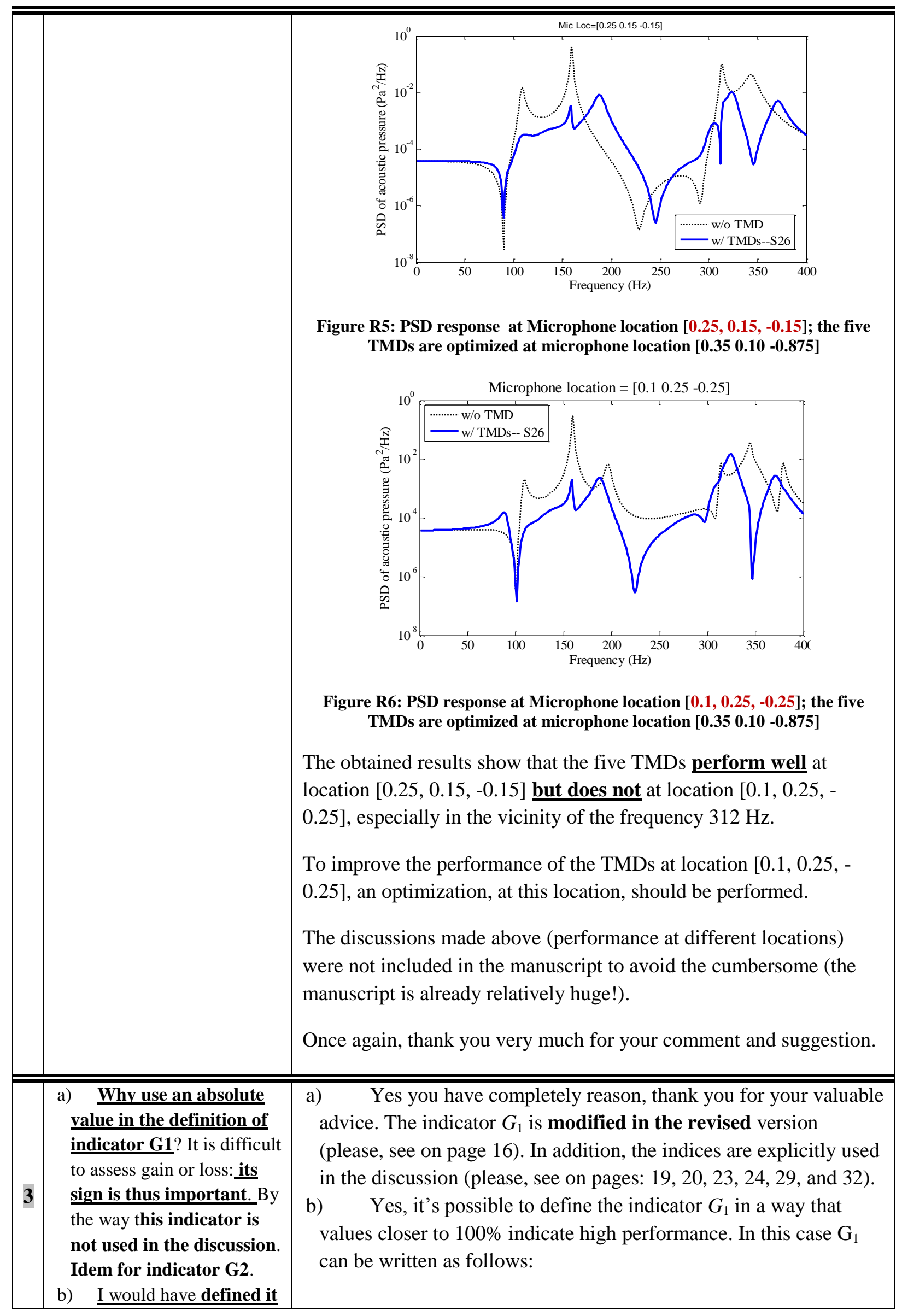




\begin{tabular}{|c|c|c|}
\hline & $\begin{array}{l}\text { the other way around to } \\
\text { make sure values closer to } \\
100 \% \text { indicate high } \\
\text { performance. } \\
\text { c) And for both } \\
\text { narrowband and broadband } \\
\text { control, I would have } \\
\text { frequency band averaged } \\
\text { these indicators (over the } \\
\text { frequency band of interest) } \\
\text { to show the global } \\
\text { performance which is } \\
\text { more representative. Please } \\
\text { comment? }\end{array}$ & $\begin{array}{l}G_{1}=\frac{P S D\left(\varpi_{i}\right)-\max _{\omega}\left(P S D(\omega)_{\text {with TMD }}\right)}{P S D\left(\varpi_{i}\right)} \times 100 \text {; when } \\
\max _{\omega}\left(P S D(\omega)_{\text {with TMD }}\right) \rightarrow 0, G_{1} \rightarrow 100 \% \text {. } \\
\text { Nevertheless, such performance index will be less visible on figures } \\
\text { (Fig.4, Fig. } 5, \ldots) \text { where it's likely more suitable to represent the } \\
\text { gain/loss in terms of magnitudes expressed in dB (as it's shown in } \\
\text { these figures). } \\
\text { c) The aim of the sub-section } 4.3 \text { is to analyze (by means of the } \\
\left.\text { indices } G_{1} \text { and } G_{2}\right) \text { the performance of the TMD device if one of } \\
\text { the two strategies (narrowband \& broadband controls) is used. The } \\
\text { outcome of the analysis is to decide which of the two strategies is } \\
\text { more suitable to control a given resonant coupled mode. For } \\
\text { example, it has been found that a broadband control is more } \\
\text { suitable to use when a well separated coupled mode, dominated by } \\
\text { plate mode, is to be controlled. } \\
\text { Consequently we think that it's not judicious to consider a frequency } \\
\text { band averaged because the designer has to make a particular choice } \\
\text { (narrowband or broadband control). The evaluation of the global } \\
\text { performance of the TMD device is made using the index } G_{2} \text {. }\end{array}$ \\
\hline 4 & $\begin{array}{l}\text { I agree with the majority of } \\
\text { the conclusions and physical } \\
\text { interpretations of the results. } \\
\text { I suggest however that an } \\
\text { example be presented } \\
\text { wherein the TMD are } \\
\text { undamped (which } \\
\begin{array}{l}\text { corresponds to the classical } \\
\text { use and application) }\end{array}\end{array}$ & $\begin{array}{l}\text { Thank you for your advice. An example has been added in the } \\
\text { revised version. Please, see on page } \mathbf{1 8} \text { (sub-section 4.3.1) and on } \\
\text { page } 22 \text { (sub-section 4.3.2). } \\
\text { The example (added in the revised version), shows the optimzation } \\
\text { results corresponding to the target frequency } \varpi_{1}=108.59 \mathrm{~Hz} \text {, when } \\
\text { an undamped TMD is optimized; thsese results have been } \\
\text { compared with those obtained in our work (damped TMD). The } \\
\text { results show that, for both cases (undamped and damped), the } \\
\text { optimal frequencies and locations corresponding to } \Delta f=2 \mathrm{~Hz} \text { are } \\
\text { roughly the same which is predictable since an optimized damped } \\
\text { TMD with } \xi_{T}^{*}=0.01 \% \text { is, in practical front, an undamped TMD. } \\
\text { In Table } \mathrm{R} 1 \text { (below), are also shown the optimization results for } \\
\text { broadband control ( } \Delta f=40 \mathrm{~Hz} \text { ) when both cases (undamped and } \\
\text { damped TMD) are considered. Unlike the narrowband control ( } \\
\Delta f=2 \mathrm{~Hz} \text { ), the optimal frequencies are significantly different } \\
\text { leading to different performances as shown in Figure R7. } \\
\text { Indeed, we can see in Figure } \mathrm{R} 7 \text { that the performances of the } \\
\text { undamped and damped TMD are roughly the same when a } \\
\text { narrowband control is performed ( } \Delta f=2 \mathrm{~Hz} \text { ) whereas they are }\end{array}$ \\
\hline
\end{tabular}




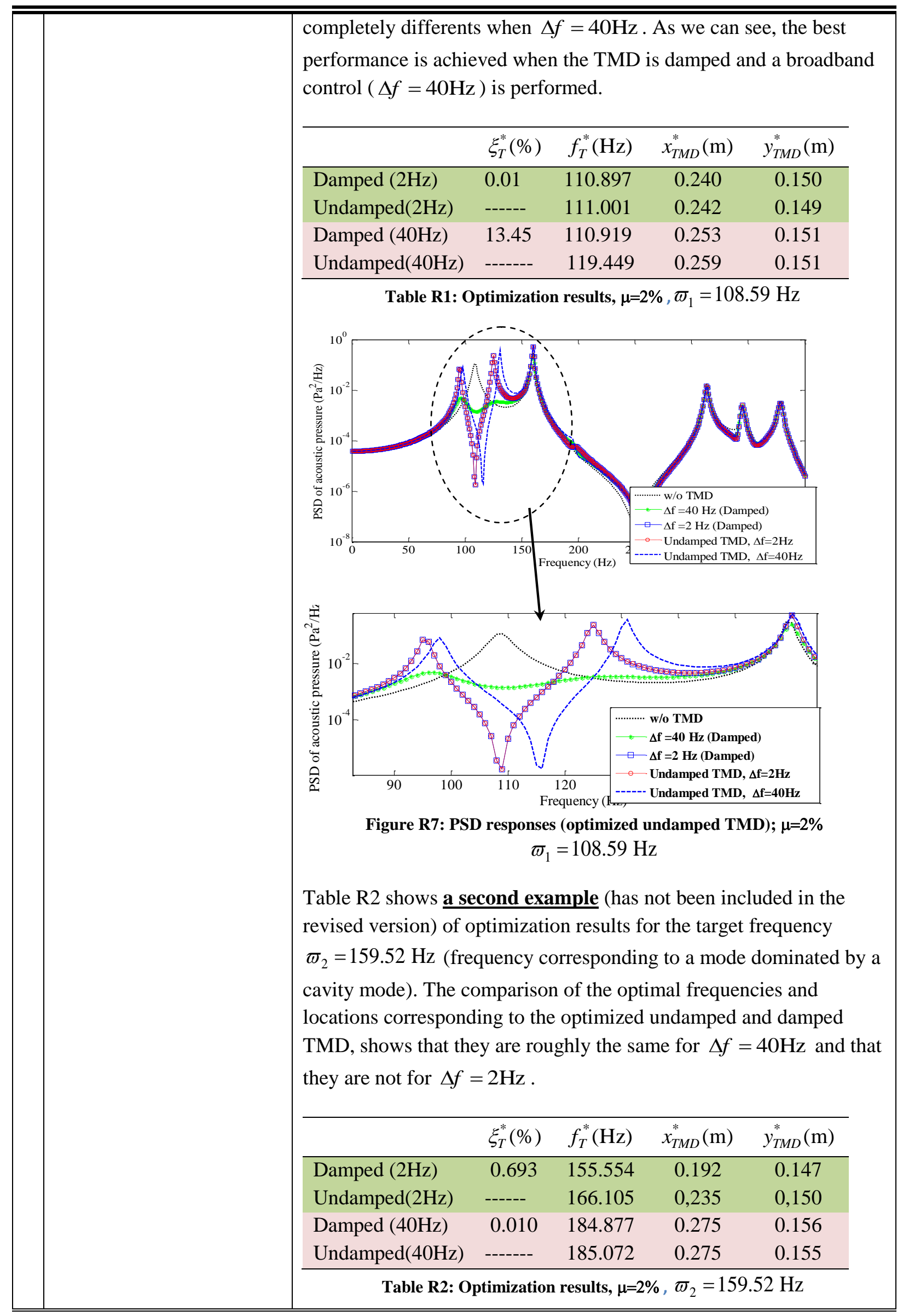




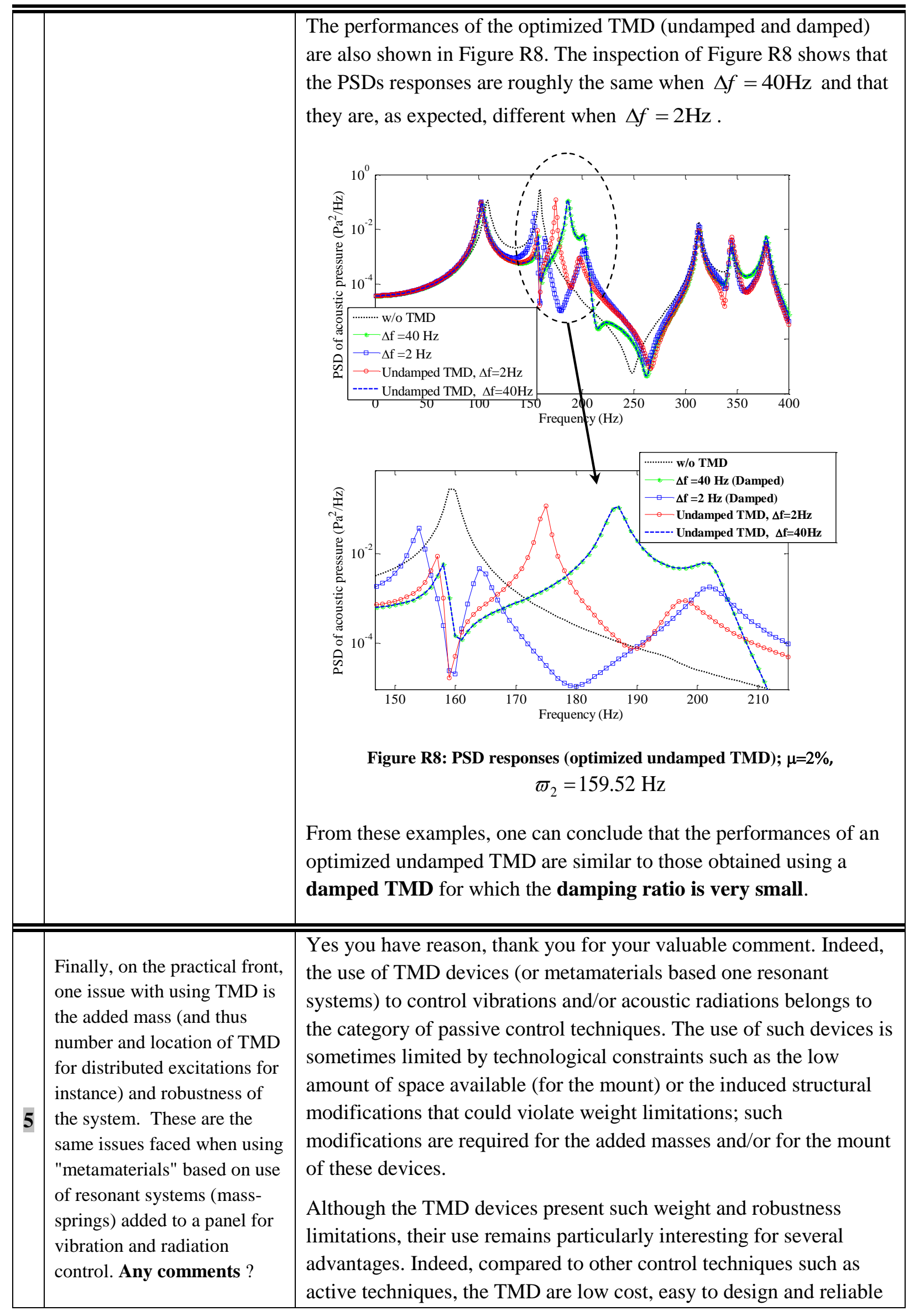




\begin{tabular}{|c|c|c|}
\hline & & devices. \\
\hline \multicolumn{3}{|r|}{ Other minor comments } \\
\hline 1 & $\begin{array}{l}\text { Add a quick justification of } \\
\text { the selected optimisation } \\
\text { algorithm (NSGA-II). }\end{array}$ & $\begin{array}{l}\text { Thank for your valuable advice. A justification is added. } \\
\text { Please, see on pages: } 13,27,30 \text { and } 34 \text {. }\end{array}$ \\
\hline 2 & $\begin{array}{l}\text { Any reasons for the selected } \\
\text { excitation and receiver } \\
\text { locations? }\end{array}$ & $\begin{array}{l}\text { The locations of the excitation and the receiver are completely } \\
\text { arbitrary. Numerical investigations have shown similar results when } \\
\text { different locations have been considered. Thank for your valuable } \\
\text { comment. }\end{array}$ \\
\hline 3 & $\begin{array}{l}\text { Explain how the frequencies } \\
\text { of the coupled modes are } \\
\text { obtained }\end{array}$ & $\begin{array}{l}\text { The frequencies of the coupled modes are obtained by means of } \\
\text { modal analysis. The coupled mass and stiffness matrices } \mathrm{M} \text { and } \mathrm{K} \text {, } \\
\text { respectively, are firstly built after truncation } \mathrm{Ns}=21 \text { and } \mathrm{Na}=102 \text {. To } \\
\text { obtain the matrices } \mathrm{M} \text { and } \mathrm{K} \text {, we can use Eq. (14) and remove the } \\
\text { terms (rows and columns) corresponding to the TMDs devices. Then } \\
\text { the eigenfrequencies are obtained using the eigen values solver of } \\
\text { Mtalab. }\end{array}$ \\
\hline 4 & $\begin{array}{l}\text { What is the effect of any of } \\
\text { the TMD on the frequencies } \\
\text { of the coupled modes. }\end{array}$ & $\begin{array}{l}\text { Thank for your valuable comment. In the present work, the TMD } \\
\text { devices specifically deal with the coupled modes rather than dealing } \\
\text { with structural (plate) modes as it's usually done in the literature. } \\
\text { Consequently, the obtained results (reduction in PSDs responses) are } \\
\text { the effects of the TMDs on the coupled modes. }\end{array}$ \\
\hline 5 & $\begin{array}{l}\text { Explain why the constant } \\
\text { pressure mode of the cavity } \\
\text { lowers rather than increases } \\
\text { the first mode of the panel. }\end{array}$ & $\begin{array}{l}\text { Thank for your valuable work. The constant pressure mode of the } \\
\text { cavity increases the first mode of the panel only if the system is } \\
\text { modeled in terms of just one uncoupled fluid mode (the first cavity } \\
\text { mode) and one uncoupled structural mode (the first plate mode). In } \\
\text { this situation, and as explained by Fahy and Gardonio (please see in } \\
\text { Ref [32], p.422-424), the first mode of the panel increases. } \\
\text { In our case, the two first modes (of plate and cavity) are also coupled } \\
\text { to the other modes via the matrix } C_{n m} \text { and the coupling effects have } \\
\text { lowered the first mode of the panel. }\end{array}$ \\
\hline
\end{tabular}

\section{Additional comments from the authors:}

A complete revision of the manuscript has been done in an attempt to clarify some statements and improve the original manuscript. The authors are grateful to the Editor in Chief and Reviewers for their valuable comments and interest in improving their 
manuscript, and hope that the revisions will satisfy the requirements to ensure the publication of their paper in "MSSP journal". 


\section{Highlights}

- Tuned Mass Dampers are used to control random interior sound;

- A stochastic acoustic optimization strategy is proposed;

- Multi-objective optimization is used along with multiple TMDs;

- The optimization strategy is able to handle multimodal control of interior sound. 


\title{
Random vibro-acoustic control of internal noise through optimized Tuned Mass Dampers
}

\author{
E. Mrabet ${ }^{(1), *}$, M. N. Ichchou ${ }^{(2)}$, N. Bouhaddi ${ }^{(3)}$ \\ (1) Laboratoire de Mécanique, Modélisation et Productique, Ecole Nationale d'Ingénieurs de Sfax, Route Soukra Km \\ 3.5 B.P 1173-3038, Sfax, Tunisie \\ (2) LTDS UMR5513 Ecole Centrale de Lyon, Université de Lyon, Ecully, France \\ (3) Univ. Bourgogne Franche-Comté, FEMTO-ST Institute, CNRS/UFC/ENSMM/UTBM, Department of Applied \\ Mechanics, 25000 BESANÇON-FR
}

\begin{abstract}
The present work deals with the passive control of interior sound pressure induced by randomly vibrating structure coupled to an enclosure filled with air. The control in the low frequency range is achieved using a Tuned Mass Damper (TMD) device. In order to guarantee an optimal performance of the TMD device, a stochastic acoustic optimization strategy is proposed. In the later, the objective function is the root mean square acoustic pressure measured at a given location inside the cavity. Assuming linear behavior of the vibro-acoustic system, the modal interaction approach is used and the objective function is evaluated by means of spectral analysis. The effectiveness of the proposed strategy is investigated and compared with others from the open literature.

The capability of the proposed optimization strategy to deal with multimodal control of interior sound has also been addressed. To this end, multiple TMDs have been used. The optimal TMD parameters have been obtained using a multi-objective optimization approach and the results that have been gathered show significant attenuations in all target resonant modes.
\end{abstract}

\section{Key words}

Interior noise control; stochastic acoustic optimization; root mean square acoustic pressure; multiple tuned mass damper; multimodal control of interior sound.

\section{Highlights}

- Tuned Mass Dampers are used to control random interior sound;

- A stochastic acoustic optimization strategy is proposed;

- Multi-objective optimization is used along with multiple TMDs;

- The optimization strategy is able to handle multimodal control of interior sound.

\section{*: corresponding author}

Elyes Mrabet; E-mail address: elyes.mrabet@isetkr.rnu.tn ; 


\section{Introduction}

Nowadays, industrial structures have become increasingly complex and their design is subject to an important number of requirements and constraints in view of improving their performance. In this context, the control of the interior sound has been considered by several research works and as a matter of fact, several techniques have been presented in the recent years. In the automotive sector [1], the comfort of the passengers is of the utmost importance and efforts have been made to reduce the interior noise induced by several sources like the engine and the tyres, among others. In the aerospace sector [2-7], C. Howard [2] reported that the excessive interior sound in the payload bays of launch vehicles causes $60 \%$ of the first day satellite failure; consequently an interior sound control of such a structure is of vital interest to minimize the failure probability.

\subsection{Overview of the existing techniques for internal noise control}

Depending on the specificities of the studied structures, the interior sound control can be achieved by different kinds of strategies [5, 7-11]: the active structural acoustic control (ASAC), the active noise control (ANC) and the passive control. Unlike the ASAC and the ANC methods, where additional sources of energy are required to achieve the sound control, the passive control techniques don't involve any external source of energy [12] and they only consist in the use of buffers, absorbers, dampers, tuned vibration absorbers (TVA) [8, 13], and so on. For instance, the TVAs are very simple devices, composed by mass, damper and spring, that have been widely used in the field of structural vibration attenuations $[14,15]$. Depending on the application, the TVAs can be used in two ways, resulting in different optimal criteria and design requirements [13,16]: (1) they can be tuned to suppress the vibration at a specific troublesome excitation frequency and in this case the TVA referred to as a tuned vibration neutralizer (TVN); (2), they can be tuned to dampen the modal contribution from a specific troublesome natural frequency of the controlled vibrating structure, and in this case the TVA referred to as a TMD.

For an optimal performance of the TVN, its natural frequency should be tuned to the excitation frequency and its damping should be as low as possible [17]. The optimal performance of the TMD device is reached when an appropriate optimization is performed allowing obtaining an optimal natural frequency, slightly lower than that of a targeted mode of the vibrating structure, and an optimal damping ratio [17].

The TMDs are particularly useful when the excitation has a broad frequency spectrum and they are used to dampen a particular resonance peak of the frequency response function relating the structural response to the excitation [16]. The potential of the TMD devices, in structural vibration mitigation, are recognized and well established since the pioneer work of Den Hartog [18]. Since a few years, the performance of the TMD devices, in sound control, has been investigated [19] and satisfactory results have been established particularly for the thin-walled enclosure cavity systems [2, 20-23].

Although the capability of the TMD device has been shown, in the interior sound control, the use of such device is very often associated with the use of a Helmholtz resonator [4, 20, 24-26], which constitutes the acoustic equivalent TMD device. Indeed, when dealing with flexible vibrating structures weakly coupled to an enclosure cavity, the obtained resonant coupled modes are either controlled (or dominated) by structure modes or by cavity modes $[27,28]$. When the acoustic coupled resonant modes are controlled by the structure, TMDs attached to the flexible structure are tuned to these modes. On the contrary, if the 
acoustic resonant modes are controlled by the cavity, Helmholtz resonators, placed into the cavity, are used to achieve the interior sound control. Recently, authors in Ref. [2] used a single device constituted by a combination of the two devices (called a passive vibro-acoustic device); the combined devices are installed on the walls of the fairing of the payload bay of a space launch vehicle and the reduction of the internal sound is achieved.

Eventhough the TMD devices present suitable solutions to structural vibration reductions, their uses in a context of interior sound control remains relatively weak due to the engineers' perception that such devices could be used only for structural vibration mitigations. Indeed, Wright and Kidner in [8] wrote : "Surprisingly few installations of vibration absorbers for interior noise control have been realized, and we believe this is due to the pervasive belief in industry that vibration absorbers can only be applied to control resonant, not forced behavior in a structure".

The review of the literature shows that the "pervasive belief in industry", as has been reported in Ref. [8], is actually not very surprising because of the lack of specific design criteria dedicated to the TMD's parameters optimization in a context of interior sound control. In this framework, one of the contributions of this paper is to propose a specific optimization criterion dealing with the TMDs parameters optimization in a context of interior sound control.

\subsection{Optimization strategies of the TMD parameters for internal sound attenuation}

Since the work of Den Hartog [18], the TMD has mainly been used for the passive control of structural vibrations. The performance of these devices deeply depends on their parameters that should be carefully assigned by performing judicious optimizations. Depending on the nature of the external excitation and the structural response parameters [29-31], several optimization strategies can be found in the literature, and one can distinguish the strategies that are deterministic (where the excitation is deterministic) and those that are stochastic (i.e. random external excitation).

A classical tuning of TMD parameters is the one proposed by Den Hartog [18] and is obtained by performing deterministic optimization strategy. The optimal TMD parameters proposed by Den Hartog are the natural frequency $\omega_{T}^{*}$ and the damping ratio $\xi_{T}^{*}$ defined by $\omega_{T}^{*}=\varpi /\left(1+\mu_{\text {eff }}\right)$ and $\xi_{T}^{*}=\sqrt{3 \mu_{e f f} / 8\left(1+\mu_{e f f}\right)}$; where $\varpi$ is the targeted frequency and $\mu_{e f f}$ is the effective mass ratio as defined by Warburton in [29, 30].

Unlike Den Hartog, who considered harmonic excitation, Warburton [29, 30] considered random excitation applied to a vibrating structure and deduced the optimal TMD parameters given by $\omega_{T}^{*}=\varpi \times \sqrt{\left.1+\mu_{\text {eff }} / 2\right)} /\left(1+\mu_{\text {eff }}\right) \quad$ and $\quad \xi_{T}^{*}=\sqrt{\mu_{e f f}\left(1+3 \mu_{e f f} / 4\right) / 4\left(1+\mu_{e f f}\right)\left(1+\mu_{\text {eff }} / 2\right)}$. Similarly to Warburton, Korenev and Reznikov [4, 6, 32] have considered a vibrating structure submitted to a wide band random excitation and developed an easy-to-use formula providing the optimal TMD parameters given as $\omega_{T}^{*}=\varpi$ and $\xi_{T}^{*}=\sqrt{\mu_{e f f}\left(1+0.75 \times \mu_{e f f}\right) / 4\left(1+\mu_{e f f}\right)^{3}}$.

Although the aforementioned optimal tuning parameters are suitable in structural vibration mitigations, their use in a context of interior sound control [4] could be inappropriate. Indeed, when dealing with interior sound control, the fluid-structure coupling effects should be taken into account. Besides, such 
optimal TMD parameters, based on structural responses, are basically valid for the mitigation of the resonant coupled modes that are controlled by the vibrating structure; the use of a TMD device with resonant modes that are controlled by the cavity may not be possible because it's possible that there is no corresponding structural resonant mode.

In contrast with the aforementioned optimization strategies, where only structural responses have been considered to deduce optimal TMD parameters, Fuller et al. [5] and Howard et al. [20] used cost functions related to the interior Acoustic Potential Energy (APE) to deduce optimal TMD parameters. Eventhough the APE based strategies used in $[2,5,20]$ constitute acoustic criteria based optimizations, they can be categorized as deterministic optimization strategies since they consider a harmonic loading applied to the vibro-acoustic system; consequently, such criteria could be inappropriate when dealing with random mechanical loading applied to a vibro-acoustic system.

\subsection{Main contributions and organization of the present work}

In the work presented here, a flexible thin structure weakly coupled to an enclosure cavity is considered and the interior sound pressure is controlled, in the low frequency range, using TMDs. The considered flexible structure is submitted to stochastic mechanical excitation and a stochastic acoustic optimization criterion is proposed to obtain the optimal parameters of the TMDs devices.

In the proposed optimization strategy, the objective function is the root mean square acoustic pressure at a given location inside the cavity. The evaluation of the objective function is performed using spectral analysis by assuming linear behavior of the vibro-acoustic system; thus the modal interaction approach [33] can be used and the optimization is carried out.

The effectiveness of the proposed strategy is demonstrated when dealing with the control of single coupled modes by performing single-objective optimizations. The obtained results show that a TMD device, alone, can always be used to control both kinds of coupled modes (i.e. those that are controlled by the structure and those controlled by the cavity); thus there is no need to use a Helmholtz resonator as it's usually proposed in the literature. Besides, comparisons with other optimization strategies have been carried out and the superiority of the proposed strategy is highlighted.

To show the capability of the proposed optimization strategy to deal with multimodal control of interior sound, multiple TMDs devices have been used to control the interior sound pressure. Rather than performing single-objective optimization, the optimal TMDs parameters are obtained using a multiobjective optimization [31, 34-40] and the obtained results show significant attenuations of the interior sound pressure in the vicinity of the targeted coupled modes.

The present paper is organized as follows: in section 2, the governing equations describing the dynamic of the vibro-acoustic system are presented. In section 3, the proposed stochastic acoustic optimization strategy is formulated. In section 4, a numerical example is analyzed and the performance of the proposed strategy is investigated. Eventually, some relevant conclusions are drawn in section 5. 


\section{Governing equations}

Consider the acoustic-structural system shown in Fig. 1. In this system, a simply supported plate is coupled to a cavity filled with air (light fluid). The acoustic cavity has five rigid walls and the flexible plate is submitted to a point force $F_{z}$ modelled as a stationary zero mean Gaussian white noise. In order to control the interior noise in the low frequencies range, multiple TMD's devices are attached to the vibrating plate (Fig. 1). The force location is defined by coordinates $\mathbf{r}_{F}=\left(x_{F}, y_{F}\right)^{\mathrm{T}}$ whereas the TMDs locations are defined by $\mathbf{r}_{c_{j}}=\left(x_{c_{j}}, y_{c_{j}}\right)^{\mathrm{T}}, j=\{1,2, . ., N\}$, the superscript in $\left({ }^{*}\right)^{\mathrm{T}}$ denotes the transpose, and $N$ is the total number of TMDs. Besides, the mass of a TMD is $m_{T_{j}}$, its damping coefficient is $c_{T_{j}}$ and its stiffness is $k_{T_{j}}$. The cavity has dimensions $l_{x}, l_{y}$ and $l_{z}$, whereas the plate has dimensions $l_{x}$ and $l_{y}$ as shown in Fig. 1.

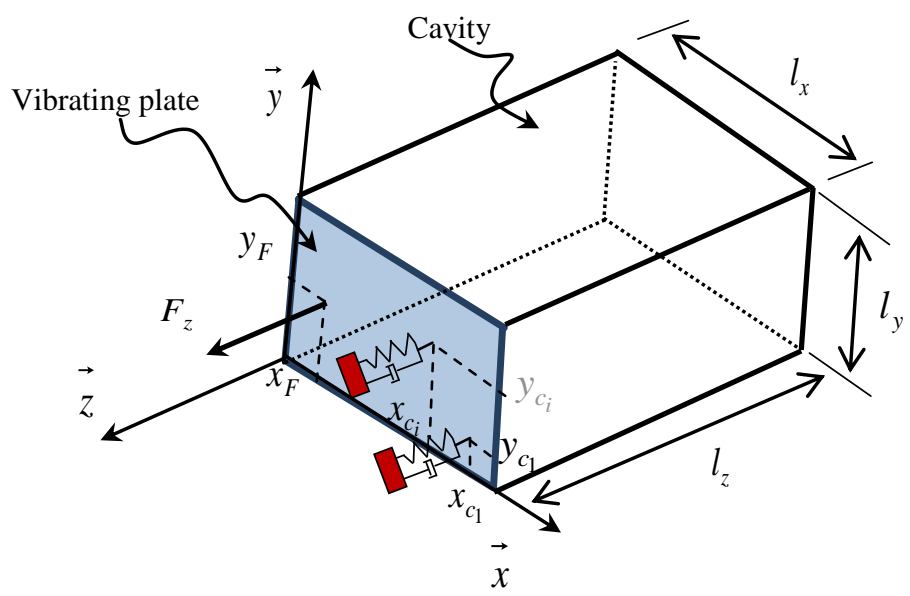

Fig. 1: Plate-cavity system with the attached multi-TMDs devices

Assuming linear behavior of the entire system, the modal coupling approach can be used. In the following sub-sections, the governing equation of the plate-cavity sub-system is first derived and then the effects of the TMDs devices are incorporated.

\section{$2.1 \quad$ Plate-cavity modal coupling}

The governing equations describing the dynamic of the plate-cavity system have been established in several references in the literature and readers are referred to Ref. [33, 41], for further details. Let $\phi_{m}\left(\mathbf{r}_{s}\right)$ be the in-vacuo $m^{\text {th }}$ mode shape of the plate, calculated at vector location $\mathbf{r}_{s}$, and denote $\omega_{m}$ its corresponding natural frequency. The plate displacement (deflection) at a given time $t$ can be written in terms of a summation over the in-vacuo normal modes as follows: 
$w\left(\mathbf{r}_{s}, t\right)=\sum_{m=1}^{N s} w_{m}(t) \phi_{m}\left(\mathbf{r}_{s}\right)$

where $N s$ is the number of elastic modes considered in the analysis, and $w_{m}(t)$ is the modal participation factor of the $m^{\text {th }}$ mode shape of the plate.

For the simply supported plate considered in the present work, the natural frequencies can be obtained as follows [42]:

$\omega_{m}\left(m_{1}, m_{2}\right)=\sqrt{E h^{3} / 12\left(1-v^{2}\right) h \rho_{s}} \times\left(\left[\frac{m_{1} \pi}{l_{x}}\right]^{2}+\left[\frac{m_{2} \pi}{l_{y}}\right]^{2}\right)$,

where $m_{1}$ and $m_{2}$ are the modal indices along the axes $x$ and $y ; E, \rho_{s}, v$ and $h$ are the Young's modulus of the plate, the plate material mass density, the Poisson's ratio and the plate thickness, respectively.

The mode shapes of the plate calculated at vector location $\mathbf{r}_{s}=(x, y)^{\mathrm{T}}$ can be expressed as follows [14, 41, 42]:

$\phi_{\left(m_{1}, m_{2}\right)}(x, y)=\sin \left(\frac{m_{1} \pi}{l_{x}} x\right) \times \sin \left(\frac{m_{2} \pi}{l_{y}} y\right)$.

Similarly to the plate, at a certain vector location $\mathbf{r}_{a}=(x, y, z)^{\mathrm{T}}$, the acoustic pressure $p\left(\mathbf{r}_{a}, t\right)$ inside the cavity can be expressed in terms of a summation of its modal participation factors $p_{n}(t)$ multiplied by the $n^{\text {th }}$ acoustic modes (with rigid boundaries) $\psi_{n}\left(\mathbf{r}_{a}\right)$, corresponding to its natural frequency $\omega_{n}$. Hence,

$p\left(\mathbf{r}_{a}, t\right)=\sum_{n=1}^{N a} p_{n}(t) \psi_{n}\left(\mathbf{r}_{a}\right)$

where $\mathrm{Na}$ is number of acoustic modes considered in the analysis. The natural frequencies of the rectangular cavity, with rigid-walls boundaries, is calculated as follows:

$\omega_{n}\left(n_{1}, n_{2}, n_{3}\right)=\pi c_{0} \times \sqrt{\left(n_{1} / l_{x}\right)^{2}+\left(n_{2} / l_{y}\right)^{2}+\left(n_{3} / l_{z}\right)^{2}}$

where $n_{1}, n_{2}$ and $n_{3}$ are the modal indices along the axes $x, y$ and $z ; c_{0}$ is the speed of sound in air.

The acoustic mode shape functions are given by: 
$\psi_{\left(n_{1}, n_{2}, n_{3}\right)}(x, y, 0)=\cos \left(n_{1} \pi x / l_{x}\right) \times \cos \left(n_{2} \pi x / l_{y}\right) \times \cos \left(n_{3} \pi x / l_{z}\right)$.

Assuming proportional damping; for a given mode $m$, the equation of motion of the plate coupled with the enclosure cavity can be written in modal coordinates as follows [33, 41, 43]:

$\ddot{w}_{m}+2 \xi_{m} \omega_{m} \dot{w}_{m}+\omega_{m}^{2} w_{m}=\frac{S}{\Lambda_{m}} \sum_{n=1}^{N a} p_{n} C_{n m}+F_{m} / \Lambda_{m}$,

where $\xi_{m}$ is the damping ratio of the $m^{\text {th }}$ plate mode, $S$ is the coupling surface, $S=l_{x} \times l_{y}, \Lambda_{m}$ is the modal mass of the plate, $F_{m}$ is the modal force applied to the structure for the $m^{\text {th }}$ mode shape and $C_{n m}$ is the dimensionless coefficient describing the coupling between the $m^{\text {th }}$ plate mode and $n^{\text {th }}$ cavity mode. For a simply supported plate, the modal mass is given by $\Lambda_{m}=\rho_{s} h l_{x} l_{y} / 4$ [41] whereas the modal force is $F_{m}=F_{z} \phi_{m}\left(x_{F}, y_{F}\right), F_{z}$ is the magnitude of the force applied at $\left(x_{F}, y_{F}\right)$, as shown in Fig. 1 .

The coupling coefficient $C_{n m}$ for the simply supported plate is given by [41]:

$C_{n m}=\frac{1}{\pi^{2}} \times\left(\frac{m_{1} \times\left((-1)^{n_{1}+m_{1}}-1\right)}{n_{1}^{2}-m_{1}^{2}}\right) \times\left(\frac{m_{2} \times\left((-1)^{n_{2}+m_{2}}-1\right)}{n_{2}^{2}-m_{2}^{2}}\right)$,

In the absence of modal acoustic source excitation and assuming proportional damping [27, 44] in the air cavity, the modal response $p_{n}(\omega)$, obeys to the following coupled equation:

$\ddot{p}_{n}+2 \xi_{n} \omega_{n} \dot{p}_{n}+\omega_{n}^{2} p_{n}=-\left(\frac{\rho_{0} c_{0}^{2} S}{\Lambda_{n}}\right) \sum_{m=1}^{N s} \ddot{w}_{m} C_{n m}$,

where $\xi_{n}$ is the damping ratio of the $n^{\text {th }}$ cavity mode, $\rho_{0}$ is the density of air, and the modal volume $\Lambda_{n}$ is expressed as follows [41]:

$$
\Lambda_{n}=V \times \varepsilon_{n_{1}} \varepsilon_{n_{2}} \varepsilon_{n_{3}}
$$

where $V=l_{x} \times l_{y} \times l_{z}$ and $\varepsilon_{n}=1$ if $n=0$, and $\varepsilon_{n}=1 / 2$ if $n>0$.

\section{$2.2 \quad$ Multiple TMDs-plate-cavity coupling}

When a TMD is attached to the flexible plate at location $\left(x_{c_{j}}, y_{c_{j}}\right), j=\{1,2, \ldots, N\}$, the equation of motion of the attached mass in physical coordinate is given by [45]: 


$$
m_{T_{j}} \ddot{z}_{T_{j}}+c_{T_{j}}\left(\dot{z}_{T_{j}}-\boldsymbol{\varphi}_{c_{j}} \dot{\mathbf{w}}\right)+k_{T_{j}}\left(z_{T_{j}}-\boldsymbol{\varphi}_{c_{j}} \mathbf{w}\right)=0,
$$

where $z_{T_{j}}, \dot{z}_{T_{j}}$ and $\ddot{z}_{T_{j}}$ are the displacement, the velocity and the acceleration of the attached mass of a TMD, respectively; $\mathbf{w}$ and $\dot{\mathbf{w}}$ are the $(N s \times 1)$ vectors of the modal participation factor and their time derivative, respectively; $\boldsymbol{\varphi}_{c_{j}}$ is the $(1 \times N s)$ vector of the plate mode shapes calculated at a TMD location $\left(x_{c_{j}}, y_{c_{j}}\right)$.

The reaction force of a TMD device applied to the plate is $f_{T M D, j}=c_{T_{j}}\left(\dot{z}_{T_{j}}-\boldsymbol{\varphi}_{c_{j}} \dot{\mathbf{w}}\right)+k_{T_{j}}\left(z_{T_{j}}-\boldsymbol{\varphi}_{c_{j}} \mathbf{w}\right)$ which can be expressed in modal coordinates as follows:

$$
\left.F_{T M D, j}=\boldsymbol{\varphi}_{c_{j}}^{\mathrm{T}} f_{T M D, j}=\boldsymbol{\varphi}_{c_{j}}^{\mathrm{T}} \mid c_{T_{j}}\left(\dot{z}_{T_{j}}-\boldsymbol{\varphi}_{c_{j}} \dot{\mathbf{w}}\right)+k_{T_{j}}\left(z_{T_{j}}-\boldsymbol{\varphi}_{c_{j}} \mathbf{w}\right)\right]
$$

The total reaction forces of the multiple TMD's devices, applied to the plate, can be expressed in modal coordinates and in matrix form as follows:

$$
F_{T M D}=\sum_{j=1}^{N} \boldsymbol{\varphi}_{c_{j}}^{\mathrm{T}} f_{T M D, j}=\mathbf{\Phi}_{c}^{\mathrm{T}}\left[\mathbf{C}_{T}\left(\dot{\mathbf{z}}_{T}-\mathbf{\Phi}_{c} \dot{\mathbf{w}}\right)+\mathbf{K}_{T}\left(\mathbf{z}_{T}-\boldsymbol{\Phi}_{c} \mathbf{w}\right)\right]
$$

where $\boldsymbol{\Phi}_{c}^{\mathrm{T}}=\left[\boldsymbol{\varphi}_{c_{1}}^{\mathrm{T}} \ldots \boldsymbol{\varphi}_{c_{N}}^{\mathrm{T}}\right], \mathbf{C}_{T}=\left[\begin{array}{lll}c_{T_{1}} & & \\ & \ddots & \\ & & c_{T_{N}}\end{array}\right], \mathbf{K}_{T}=\left[\begin{array}{lll}k_{T_{1}} & & \\ & \ddots & \\ & & k_{T_{N}}\end{array}\right]$ and $\mathbf{z}_{T}=\left(z_{T_{1}} \ldots z_{T_{N}}\right)^{\mathrm{T}}$.

Rearranging Eqs. (7) and (9) in matrix form and letting $\mathbf{q}=\left(\mathbf{w}^{\mathrm{T}}, \mathbf{p}^{\mathrm{T}}, \mathbf{z}_{T}\right)^{\mathrm{T}}$ be the vector of modal coordinates, where $\mathbf{p}$ is the $(N a \times 1)$ vector of the acoustic modal participation factor. The insertion of the expression of the modal force $F_{T M D}$ into Eq. (7), yields the coupled equation of the TMD-plate-cavity system that can be expressed as follows $[14,20]$ :

$$
\mathbf{M} \ddot{\mathbf{q}}+\mathbf{D} \dot{\mathbf{q}}+\mathbf{K q}=\boldsymbol{\Phi}^{\mathrm{T}} F_{z},
$$

where $\dot{\mathbf{q}}$ and $\ddot{\mathbf{q}}$ are the time derivatives of $\mathbf{q} ; \boldsymbol{\Phi}=\left(\begin{array}{lll}\boldsymbol{\varphi}_{F} & \boldsymbol{\psi}_{0} & 0\end{array}\right), \boldsymbol{\varphi}_{F}$ is the $(1 \times N s)$ vector of the plate mode shapes computed at force location $\left(x_{F}, y_{F}\right)$ and $\boldsymbol{\psi}_{0}$ is a $(1 \times N a)$ vector of zeros;

$$
\mathbf{M}=\left[\begin{array}{ccc}
\boldsymbol{\Lambda}_{m} & \mathbf{0} & \mathbf{0} \\
S \mathbf{C}_{n m} & \frac{1}{\rho_{0} c_{0}^{2}} \boldsymbol{\Lambda}_{n} & \mathbf{0} \\
\mathbf{0} & \mathbf{0} & \mathbf{m}_{T}
\end{array}\right], \boldsymbol{\Lambda}_{m}=\left[\begin{array}{lll}
\ddots & & \\
& \Lambda_{m} & \\
& & \ddots
\end{array}\right], \boldsymbol{\Lambda}_{n}=\left[\begin{array}{lll}
\ddots & & \\
& \Lambda_{n} & \\
& & \ddots
\end{array}\right] ; \mathbf{m}_{T}=\left[\begin{array}{ccc}
m_{T_{1}} & & \\
& \ddots & \\
& & m_{T_{N}}
\end{array}\right] ;
$$


and $\mathbf{C}_{n m}$ is the coupling matrix containing the coupling elements $C_{n m}$.

\section{$2.3 \quad$ Spectral analysis}

When dealing with stochastic loading applied to a vibro-acoustic system, the random analysis theory can be used [46], where it's usual to characterize the responses by means of the power spectral density (PSD) functions [47] and/or by means of the covariance responses. In the present work, it's assumed that the multiple TMDs-plate-cavity system behaves linearly and the plate is excited by stationary zero mean Gaussian white noise. Consequently, the acoustic pressure response, in a given location $\mathbf{r}_{a}$ inside the cavity, is also a zero mean Gaussian process that can be characterized by its PSD and/or its root mean square value.

Let $\widetilde{\mathbf{q}}(\omega), \widetilde{\mathbf{w}}(\omega), \widetilde{\mathbf{p}}(\omega)$ and $\widetilde{\mathbf{z}}_{T}(\omega)$ be the finite Fourier transform of $\mathbf{q}, \mathbf{w}, \mathbf{p}$ and $\mathbf{z}_{T}$, respectively. Similarly to Eqs.(1) and (4), the Fournier transform of $w\left(\mathbf{r}_{s}, t\right)$ and $p\left(\mathbf{r}_{a}, t\right)$ can be expanded in terms of $\tilde{\mathbf{w}}(\omega), \tilde{\mathbf{p}}(\omega)$ and written in vector form as follows:

$\widetilde{w}\left(\mathbf{r}_{s}, \omega\right)=\boldsymbol{\varphi}\left(\mathbf{r}_{s}\right) \tilde{\mathbf{w}}(\omega)$ and $\tilde{p}\left(\mathbf{r}_{a}, \omega\right)=\boldsymbol{\psi}\left(\mathbf{r}_{a}\right) \tilde{\mathbf{p}}(\omega)$,

where $\boldsymbol{\psi}\left(\mathbf{r}_{a}\right)$ is the $(1 \times N a)$ vector of the acoustic mode shape calculated at location $\mathbf{r}_{a}$. Besides, let $\tilde{F}_{z}$ be the Fourier transform of the excitation force $F_{z}$. The application of the Fourier transform for both sides of Eq. (14) yields the following expression:

$\widetilde{\mathbf{q}}=\left(-\omega^{2} \mathbf{M}+j \omega \mathbf{D}+\mathbf{K}\right)^{-1} \mathbf{\Phi}^{\mathrm{T}} \tilde{F}_{z}$

and the modal acoustic pressure $\widetilde{\mathbf{p}}(\omega)$ is deduced as follows:

$\tilde{\mathbf{p}}(\omega)=\mathbf{Y} \boldsymbol{\Phi}^{\mathrm{T}} \tilde{F}_{z}$,

where $\mathbf{Y}$ is the $N a \times(N s+N a+N)$ sub-matrix extracted from the matrix $\left(-\omega^{2} \mathbf{M}+j \omega \mathbf{D}+\mathbf{K}\right)^{-1}$ by taking the $N a$ rows corresponding to the modal acoustic pressure $\tilde{\mathbf{p}}(\omega)$.

Using Eq. (17), the PSD matrix [7, 48] of the modal acoustic pressure $\widetilde{\mathbf{p}}(\omega)$ can be obtained as follows: 


$$
S_{\tilde{\mathbf{p}} \tilde{\mathbf{p}}}(\omega)=\lim _{T \rightarrow \infty} \frac{1}{2 T} E\left\{\tilde{\mathbf{p}}(\omega) \tilde{\mathbf{p}}^{*}(\omega)\right\}=\lim _{T \rightarrow \infty} \frac{1}{2 T} E\left\{\mathbf{Y} \boldsymbol{\Phi}^{\mathrm{T}} \tilde{F}_{z} \tilde{F}_{z}^{*} \mathbf{\Phi} \mathbf{Y}^{*}\right\}=\mathbf{Y} \boldsymbol{\Phi}^{\mathrm{T}} S_{F F} \boldsymbol{\Phi} \mathbf{Y}^{*}
$$

where (*) denotes the complex conjugate, $E\{$.$\} is the expectation operator and S_{F F}=\lim _{T \rightarrow \infty} \frac{1}{2 T} E\left\{\tilde{F}_{z} \tilde{F}_{z}^{*}\right\}$ is the constant PSD of the Gaussian white noise excitation applied to the plate.

By making use of the expressions in Eqs. (15), (17) and (18), the PSD $S_{\tilde{p} \tilde{p}}\left(\mathbf{r}_{a}, \mathbf{r}_{F}, \omega\right)$ of the acoustic pressure, at a given location $\mathbf{r}_{a}$ inside the cavity and for a force location $\mathbf{r}_{F}$, is given by:

$$
S_{\tilde{p} \tilde{p}}\left(\mathbf{r}_{a}, \mathbf{r}_{F}, \omega\right)=\lim _{T \rightarrow \infty} \frac{1}{2 T} E\left\{\tilde{p}\left(\mathbf{r}_{a}, \mathbf{r}_{F}, \omega\right) \tilde{p}^{*}\left(\mathbf{r}_{a}, \mathbf{r}_{F}, \omega\right)\right\}=\boldsymbol{\psi}\left(\mathbf{r}_{a}\right) \mathbf{Y}(\omega) \boldsymbol{\Phi}^{\mathrm{T}}\left(\mathbf{r}_{F}\right) S_{F F} \boldsymbol{\Phi}\left(\mathbf{r}_{F}\right) \mathbf{Y}^{*}(\omega) \boldsymbol{\psi}^{\mathrm{T}}\left(\mathbf{r}_{a}\right)
$$

Denote $H\left(\omega, \mathbf{r}_{a}, \mathbf{r}_{F}\right)=\boldsymbol{\psi}\left(\mathbf{r}_{a}\right) \mathbf{Y}(\omega) \boldsymbol{\Phi}^{\mathrm{T}}\left(\mathbf{r}_{F}\right)$, the PSD of the acoustic pressure is finally given as:

$$
S_{\tilde{p} \tilde{p}}\left(\mathbf{r}_{a}, \mathbf{r}_{F}, \omega\right)=\left|H\left(\omega, \mathbf{r}_{a}, \mathbf{r}_{F}\right)\right|^{2} S_{F F}
$$

It should be noted that the scalar quantity $H\left(\omega, \mathbf{r}_{a}, \mathbf{r}_{F}\right)=\boldsymbol{\psi}\left(\mathbf{r}_{a}\right) \mathbf{Y}(\omega) \boldsymbol{\Phi}^{\mathrm{T}}\left(\mathbf{r}_{F}\right)$ represents the acoustic pressure Frequency Response Function (FRF) measured at location $\mathbf{r}_{a}$ for a given excitation applied to the plate at location $\mathbf{r}_{F}$. The expression obtained in Eq. (20) is the classical relationship between the inputoutput PSDs $\left(S_{F F}\right.$ and $S_{\tilde{p} \tilde{p}}\left(\mathbf{r}_{a}, \mathbf{r}_{F}, \omega\right)$ ) in the random analysis theory [46]. Similarly to the pressure FRF, the plate displacement FRF at force location can be obtained from $H_{\mathrm{d}}\left(\omega, \mathbf{r}_{a}, \mathbf{r}_{F}\right)=\boldsymbol{\psi}\left(\mathbf{r}_{a}\right) \hat{\mathbf{Y}}(\omega) \boldsymbol{\Phi}^{\mathrm{T}}\left(\mathbf{r}_{F}\right)$, where $\hat{\mathbf{Y}}(\omega)$ is the $N s \times(N s+N a+N)$ sub-matrix extracted from the matrix $\left(-\omega^{2} \mathbf{M}+j \omega \mathbf{D}+\mathbf{K}\right)^{-1}$ by taking the $N s$ rows corresponding to the modal participation factors $\widetilde{\mathbf{w}}(\omega)$.

Based on the expression of the PSD $S_{\tilde{p} \tilde{p}}\left(\mathbf{r}_{a}, \mathbf{r}_{F}, \omega\right)$, one can also compute the root mean square acoustic pressure (RMSAP) $\sigma_{p}\left(\mathbf{r}_{a}, \mathbf{r}_{F}\right)$ at a given location $\mathbf{r}_{a}$, which can be expressed as follows [49, 50]:

$$
\sigma_{p}\left(\mathbf{r}_{a}, \mathbf{r}_{F}\right)=\sqrt{\int_{\omega^{l}}^{\omega^{u}} S_{\tilde{p} \tilde{p}}\left(\mathbf{r}_{a}, \mathbf{r}_{F}, \omega\right) d \omega}
$$

where $\omega^{l}$ and $\omega^{u}$ are the lower and upper bounds of a certain bandwidth of interest $\Delta f=\left[\omega^{l}, \omega^{u}\right]$ centered at a given frequency $\varpi$. 


\section{The proposed optimization strategies}

Generally speaking, different approaches can be used to formulate an objective function in a structuralacoustic optimization for passive noise control. These approaches can be categorized into four groups [51, 52], the first one being the sound pressure level at one or more specified points basically utilized for closed domains [52-55]. The second group, utilized in open domains, considers the emitted sound power as objective function. The third group considers the transmission loss while the fourth one considers all the other objective functions.

As mentioned previously, under external random mechanical loading, the acoustic pressure inside the cavity, shown in Fig. 1, can be characterized by its PSD measured at a given location $\mathbf{r}_{a}$. In the low frequency range, the presence of resonant modes can be observed by the presence of PSD response peaks in the very close vicinity of the natural frequencies of the coupled vibro-acoustic system.

In Fig. 2 a schematic representation of an acoustic PSD response and two peaks, corresponding to two resonant frequencies $\left(\varpi_{1}\right.$ and $\left.\varpi_{2}\right)$, are depicted. In order to mitigate these resonant responses, multiple TMDs (in this case two TMDs) can be used. The basic idea is to use one TMD device for each one of the resonant modes and the TMDs parameters will be carefully chosen based on a stochastic acoustic optimization strategy.

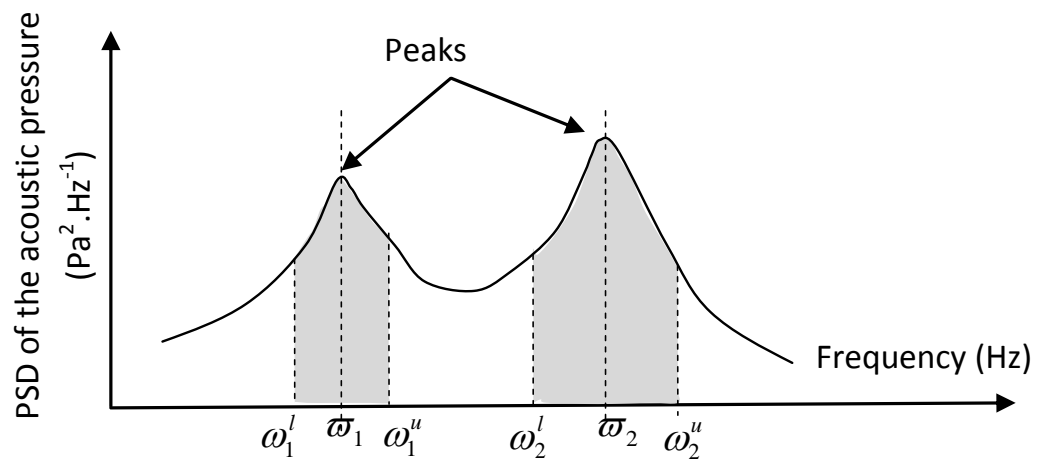

Fig. 2 : schematic representation of two objective functions (i.e. the square roots of the shaded surfaces)

In the present work, the proposed stochastic acoustic optimization strategy attempts to minimize the RMSAP evaluated, in the vicinity of a target frequency (i.e. $\varpi_{1}$ or $\varpi_{2}$ ), using Eq.(21). Since the objective function is related to a particular microphone location $\mathbf{r}_{a}$, the proposed optimization strategy belongs to the first group of optimization approaches, as described in Refs. [51, 52]. Such optimization strategy can be used to control the sound pressure at the position of the ear of a vehicle passenger/ driver $[53,55]$, for instance.

Obviously, when dealing with multiple resonant peaks the use of multiple TMDs involves simultaneous minimization of the RMSAP evaluated in the vicinity of the targeted frequencies and in this case a multiobjective optimization [34, 38, 39, 56] should be carried out. Figure 2 shows two schematic objective functions (the shaded area centered at the targeted frequencies) corresponding to resonant peaks. 
Generally, there are three parameters by which a TMD device can be characterized [8]. The first parameter is the mass ratio $\mu_{j}=m_{T_{j}} / m_{\text {plate }}$ which is the ratio between its mass and the mass of the plate ( $\left.m_{\text {plate }}=S \times h \times \rho_{s}\right)$, the others are its natural frequency $\omega_{T_{j}}=\sqrt{k_{T_{j}} / m_{T_{j}}}$ and its damping ratio $\xi_{T_{j}}=c_{T_{j}} / 2 m_{T_{j}} \omega_{T_{j}}$. Besides, since the performance of the TMD device strongly depends on its location $\mathbf{r}_{c_{j}}$ at the vibrating plate [57], the TMD location will also be optimized.

In the work conducted here, two optimization problems will be considered: the first one is the singleobjective problem where only one resonant peak is controlled using one TMD; and the second is the multi-objective problem involving multiple TMDs devices allowing a multimodal control of interior sound.

\subsection{The single-objective optimization strategy}

For the single-objective optimization, one TMD device is used to control a given resonant peak. For this problem the mass ratio will be taken constant (commonly assigned as 1 to 5\%) and the optimization problem is formulated as follows:

Find $\quad \mathbf{d}=\left(\omega_{T}, \xi_{T}, \mathbf{r}_{c}\right)^{\mathrm{T}}$ to minimize $\sigma_{p}\left(\mathbf{r}_{a}, \mathbf{r}_{F}, \mathbf{d}\right)=\sqrt{\int_{\omega^{l}}^{\omega^{u}} S_{\tilde{p} \tilde{p}}\left(\mathbf{r}_{a}, \mathbf{r}_{F}, \mathbf{d}, \omega\right) d \omega}$

Obviously, when a TMD is targeted to a particular resonant frequency, the optimal natural frequency of the TMD will be bounded in the vicinity of the frequency of interest and then the optimization problem will be constrained in frequencies. In addition, the design parameter $\mathbf{r}_{c}$ is bounded by physical limitations (plate dimensions) therefore the above optimization problem will also be constrained in TMD locations.

For the optimization problem defined in Eq. (22), it's also clear that the bandwidth parameter $\Delta f=\left[\omega^{l}, \omega^{u}\right]$ defined in Eq. (21) plays an important role in the evaluation of the objective function. Depending on this bandwidth parameter, to be considered in the optimization loop, two kinds of control will be defined: (1) the first one is the narrowband control and, (2) the second one is the broadband control. A narrowband control is performed when the bandwidth parameter $\Delta f$ is small whereas when it's large, the control is considered to be broadband.

The proposed optimization problem can be categorized as a stochastic vibro-acoustic optimization problem, since the objective functions involve covariance term related to the acoustic pressure inside the cavity.

\subsection{The multi-objective optimization strategy}

Unlike the single-objective optimization case, the proposed optimization strategy for the multi-objective optimization strategy consists in finding the optimal design vector $\mathbf{d}=\left(\mathbf{d}_{1}, \mathbf{d}_{2}, \ldots, \mathbf{d}_{N}\right)^{\mathrm{T}}$, where $\mathbf{d}_{j}=\left(\omega_{T_{j}}, \xi_{T_{j}}, \mu_{j}, \mathbf{r}_{c_{j}}\right)^{\mathrm{T}} \quad, j=\{1,2, . ., N\}$, which minimizes a vector of objective functions 

Each one of the considered objective functions will be separately evaluated in the vicinity of the targeted frequency of interest.

The proposed optimization problem can be categorized as a stochastic vibro-acoustic multi-objective optimization problem, since the objective functions involve covariance terms related to the acoustic pressures inside the cavity. The multi-objective optimization strategy can be formulated as follows :

$\operatorname{minimize} \boldsymbol{\sigma}_{p}\left(\mathbf{r}_{a}, \mathbf{r}_{F}, \mathbf{d}\right)=\left(\sigma_{p 1}, \sigma_{p 2}, \ldots, \sigma_{p N}\right)^{\mathrm{T}}$

subject to $\quad \sum_{i=1}^{N} \mu_{i} \leq \mu_{a d m}$

where $\mu_{a d m}$ is a given mass ratio defined in a pre-design phase. Similarly to the single-objective case, the above optimization problem will also be constrained in TMD locations $\left(\mathbf{r}_{c_{j}}\right)$ and in frequencies ranges, since each of the TMD devices will be tuned in the vicinity of the targeted frequencies.

Rather than obtaining a single optimal solution (i.e. for the single-objective optimization problem), a set of optimal solutions, called Pareto optimal solutions [34-36], is obtained in the case of a multi-objective optimization problem. Several methods can be found in the literature to solve such a problem [38] and in the work presented here, a controlled elitist Genetic Algorithm (GA), which is a variant of the Nondominated Sorting Genetic Algorithm II (NSGA-II) [37, 39, 56], has been used. The algorithm is already implemented in Matlab and it has the advantage to favor individuals that can help increase the diversity of the population even if they have a lower fitness value. It should be noted that it's important to maintain the diversity of population for convergence to an optimal Pareto front.

The Pareto optimal solutions obtained using the controlled elitist GA constitutes a trade-off between the objective functions. In addition, it's well known that the determination of a particular solution among the set of solutions is a difficult task, especially when dealing with a great number of objective functions (more than three dimensions). Several techniques can be found in the literature [34, 36, 58-60] to help the decision-maker, and among them one can find the Self-Organizing Maps (SOM) introduced by Kohonen $[61,62]$. The SOM is an unsupervised neural network algorithm providing clusters based on similarities between the optimal solutions; these clusters are represented in two-dimensional maps allowing easier visualization of the Pareto data. The reader is referred to [61, 62] for further details about the SOM algorithm and its applications. In the work conducted here, the SOM toolbox available in Ref. [63] has been used.

\section{Numerical example}

The aim of this section is to investigate the efficiency of the proposed optimization strategy in the sound attenuation of a plate-cavity system when it's submitted to mechanical stochastic loading. The investigations are firstly performed when a single-objective optimization, involving the use of one TMD, is considered to separately control the resonant coupled modes; and secondly a multimodal sound control, involving multiples TMDs and multi-objective optimization, is also carried out. 

Thus, one of the aims of the investigations, related to the single-objective optimization, is to determine what kind of control should be used. In other words, what values of $\Delta f$ should be assigned in order to effectively attenuate the resonant modes. If the obtained $\Delta f$ is large, the control is broadband, otherwise, it is narrowband. The obtained values of $\Delta f$, yielding good performance in the single-objective optimization, will then be used for the multimodal sound control using multiple TMDs.

The considered vibro-acoustic system is that shown in Fig. 1 and the parameter values are taken equal to those presented by Howard and Cazzolato in Ref. [41]; the dimensions and the properties of the studied system are given in Table 1. In addition, it's assumed that the observation location of the acoustic pressure (i.e. microphone location) in the cavity is located at the coordinates $\mathbf{r}_{a}=(0.35,0.1,-0.875)^{\mathrm{T}}$.

For the introduced proportional damping in both plate and cavity, the damping ratio for a mode $i$ is set to $\xi_{i}=\alpha / 2 \omega_{i}+\beta \omega_{i} / 2$ [27], where $\alpha$ and $\beta$ are two coefficients to be calculated by imposing a targeted value $\xi_{0}$ for the two frequencies bounds of the range of interest. For the plate, the targeted damping ratio is set to $\xi_{0}^{\text {plate }}=2 \%$ whereas it's set to $\xi_{0}^{\text {cavity }}=0.5 \%$ for the cavity. The PSD of the point force applied to the plate is set to $S_{F F}=0.1 \mathrm{~N}^{2} \times \mathrm{Hz}^{-1}$.

\begin{tabular}{cccc}
\hline Parameter & & Value \\
\cline { 1 - 1 }$l_{x}(\mathrm{~m})$ & & $0.5 \mathrm{~m}$ \\
$l_{y}(\mathrm{~m})$ & & $0.3 \mathrm{~m}$ \\
$l_{z}(\mathrm{~m})$ & & $1.1 \mathrm{~m}$ \\
$h(\mathrm{~m})$ & & $0.003 \mathrm{~m}$ \\
$E(\mathrm{~Pa})$ & & $70 \times 10^{9} \mathrm{~Pa}$ \\
$\rho_{s}$ (unit) & & $2700 \mathrm{~kg} \cdot \mathrm{m}^{-3}$ \\
$v$ & & 0.3 \\
$\rho_{0}$ (unit) & & $1.21 \mathrm{~kg} \cdot \mathrm{m}^{-3}$ \\
$c_{0}$ (unit) & & $344 \mathrm{~m} . \mathrm{s}^{-1}$ \\
\hline
\end{tabular}

Table 1: Numerical values of the parameters of the vibro-acoustic system

Since the control of the acoustic pressure will be performed in the low frequency range, the frequency interval of interest is set to $0-400 \mathrm{~Hz}$. Therefore only the coupled frequencies bellow $400 \mathrm{~Hz}$ have been considered in the analysis. The numerical investigations have shown that $N s=21$ and $N a=102$ allow obtaining accurate results when evaluating the objective functions (i.e. the root mean square pressure).

\subsection{Modal analysis of the uncontrolled plate-cavity system}

Before performing control of sound inside the rectangular cavity, it's important to analyze the plate-cavity system without the TMD devices. The analysis allows the determination of the resonant modes on which the TMDs will be tuned, on the one hand, and the determination of the modes involved in the coupling, for both plate and cavity, on the other. 
Table 2 shows the natural frequencies of the in-vacuo plate and those of the rigid-walled cavity, compared with those of the coupled system. As described by Fahy and Gardonio in Ref. [27], we can see that the coupled frequencies are sometimes controlled (or dominated) by the plate modes and sometimes by the cavity modes. By taking into account the numerical values obtained for the coupling matrix $\mathbf{C}_{n m}$ [64], one can also obtain the involved modes (i.e. in-vacuo and rigid-walled) for the coupled modes as shown in the last column of Table 2 .

The FRF for the forcing location $\mathbf{r}_{F}=(0.05,0.05)^{\mathrm{T}}$ are plotted in Fig. 3. In Fig. 3 (a) the acoustic pressure FRF is presented and Fig. 3 (b) shows the displacement FRF of the plate, at the forcing location. In Fig. 3, the resonant coupled modes with their corresponding modes involved in the coupling are also indicated. The results show that only five resonant modes, corresponding to the five peaks in Fig. 3 (a), have to be considered for the acoustic pressure control using TMDs, since the other modes don't induce resonant peaks in the sound pressure at the observed location inside the cavity.

Besides, as mentioned previously, one can distinguish between two kinds of resonant modes. The first kind corresponds to the modes controlled by the plate and those controlled by the cavity. The modes controlled by the plate are $\varpi_{1}=108.59 \mathrm{~Hz}$ and $\varpi_{4}=343.8 \mathrm{~Hz}$. The other modes, controlled by the cavity, are $\varpi_{2}=159.52 \mathrm{~Hz}, \varpi_{3}=313.20 \mathrm{~Hz}$ and $\varpi_{5}=378.61 \mathrm{~Hz}$.

\begin{tabular}{|c|c|c|c|c|c|c|}
\hline \multicolumn{2}{|c|}{ In-vacuo plate } & \multicolumn{2}{|c|}{ Rigid-walled cavity } & \multicolumn{3}{|c|}{ Coupled plate-cavity } \\
\hline Modes & $\begin{array}{c}\text { Frequencies } \\
(\mathrm{Hz})\end{array}$ & Modes & $\begin{array}{c}\text { Frequencies } \\
(\mathrm{Hz})\end{array}$ & $\begin{array}{c}\text { Frequencies } \\
(\mathrm{Hz})\end{array}$ & $\begin{array}{c}\text { Mode } \\
\text { controlled by }\end{array}$ & Modes involved \\
\hline \multirow{3}{*}{$(1,1)$} & \multirow{3}{*}{110.43} & $(0,0,0)$ & 0 & 0 & & \\
\hline & & & & 108,59 & plate & $(1,1)-(0,0,0)$ \\
\hline & & $(0,0,1)$ & 156.36 & 159,52 & cavity & $(1,1)-(0,0,1)$ \\
\hline \multirow{2}{*}{$(2,1)$} & \multirow[t]{2}{*}{198.12} & & & 196,52 & plate & $(2,1)-(1,0,0)$ \\
\hline & & $(0,0,2)$ & 312.72 & 313,20 & cavity & $(3,1)-(0,0,2)$ \\
\hline \multirow{2}{*}{$(3,1)$} & \multirow[t]{2}{*}{344.27} & & & 343,80 & plate & $(3,1)-(0,0,2)$ \\
\hline & & $(1,0,0)$ & 344.00 & 344,45 & cavity & $(2,1)-(1,0,0)$ \\
\hline \multirow{2}{*}{$(1,2)$} & \multirow{2}{*}{354.01} & & & 352,35 & plate & $(1,2)-(0,1,0)$ \\
\hline & & $(1,0,1)$ & 37786 & 378,61 & cavity & (21)-(1. \\
\hline
\end{tabular}

Table 2: Natural modes and frequencies of the uncoupled and coupled plate-cavity system

\subsection{TMD performance comparisons with other optimization strategies}

For the coupled modes controlled by the plate, the performance of the TMD device, when it's tuned using the proposed strategy, will be compared with its performance if it were tuned using other strategies used in the literature. In the present work, three optimization strategies will be considered: the first two strategies are those proposed by Korenev and Reznikov [4, 6], and by Den Hartog [18]; the third strategy is based on the time average APE $[2,4,5,65]$. It should be noted that the first two strategies involve structural vibrations optimization criteria (stochastic and deterministic optimizations, respectively), whereas the last one constitutes an acoustic criterion. For the coupled resonant modes controlled by cavity modes, the performance of the proposed strategy will be compared only with the strategy based on the APE, since no resonant structural frequency can be associated to these coupled modes (i.e. controlled by cavity modes). 

$E_{p}(\omega)=\frac{1}{4 \rho_{0} c_{0}^{2}} \tilde{\mathbf{p}}^{*}(\omega) \boldsymbol{\Lambda}_{n} \tilde{\mathbf{p}}(\omega)$. Consequently the optimization problem, involving an acoustic criterion, consists in finding $\mathbf{d}=\left(\omega_{T}, \xi_{T}, \mathbf{r}_{c}\right)^{\mathrm{T}}$ minimizing $E_{p}\left(\mathbf{r}_{F}, \mathbf{d}, \varpi\right)=\frac{1}{4 \rho_{0} c_{0}^{2}} \tilde{\mathbf{p}}^{*}\left(\mathbf{r}_{F}, \mathbf{d}, \varpi\right) \boldsymbol{\Lambda}_{n} \tilde{\mathbf{p}}\left(\mathbf{r}_{F}, \mathbf{d}, \varpi\right)$. It should be noted that the acoustic cost function, involving the APE, is used when a harmonic analysis (deterministic analysis) is conducted; therefore the optimization problem is considered as a deterministic one.

In addition, in order to evaluate the performance of the TMD device, when it's tuned using the proposed strategy, the PSDs responses of the acoustic pressure will be presented for each one of the targeted frequencies. For these PSDs responses, it has been assumed that the vibro-acoustic system has been submitted to band limited white noise covering the frequency range [0-400] Hz. The choice of the PSDs representations instead of the FRFs representations [66] or the APE representation is justified by the fact that the objective function is explicitly related to the acoustic PSD therefore the interpretations of the obtained results will be easier.

The performance of the TMD device is evaluated using two indices:

(1) the first index quantifies the TMD performance in the close vicinity of the target frequency $\varpi_{i}$, $i=\{1,2, . ., 5\}$ and it is expressed as follows: $G_{1}(\mathrm{~dB})=20 \log _{10}\left(\frac{\operatorname{PSD}\left(\varpi_{i}\right)}{\max _{\omega}\left(\operatorname{PSD}(\omega)_{\text {with TMD }}\right)}\right)$;

where $\operatorname{PSD}\left(\varpi_{i}\right)$ is the value of the PSD response at the target frequency $\varpi_{i}$ (peak of the PSD response without TMD) and $\max _{\omega} \operatorname{PSD}(\omega)_{\text {with TMD }}$ is the maximum value of the PSD response (with TMD) measured in the close vicinity of the target frequency $\varpi_{i}$. The higher the value of the $G_{1}$ index is, the better the performance of the TMD is.

(2) the second quantifies the global performance of the TMD over the frequency range of interest and it is given as: $G_{2}(\%)=100 \times\left(\sigma_{T M D}-\sigma_{0}\right) / \sigma_{0}$, where $\sigma_{T M D}$ is the RMSAP of the controlled vibro-acoustic system (equipped by TMD), evaluated over the frequency range [0-400] Hz; $\sigma_{0}$ is the RMSAP of the uncontrolled vibro-acoustic system, evaluated over the frequency range [0-400] Hz. The lower the value of the $G_{2}$ index is, the better the global performance of the TMD device is. It should be noted that for $\mathbf{r}_{F}=(0.05,0.05)^{\mathrm{T}}$ and $\mathbf{r}_{a}=(0.35,0.1,-0.875)^{\mathrm{T}}, \sigma_{0}=1.36 \mathrm{~Pa}$.

When comparing the performance of the proposed optimization strategy with the aforementioned strategies, three criteria are considered:

- The attenuation in the close vicinity of the target frequency should be important;

- When using the TMD device, two new peaks appear in the close vicinity of the target frequency; the magnitudes of these peaks should be as low as possible;

- The increases on the PSD responses, on the off-target frequencies, should be as low as possible. 
Tables 3 and 4 present the optimal TMD parameters when the target frequencies $\varpi_{1}$ and $\varpi_{4}$ are separately controlled, respectively. These tables are obtained for different bandwidth parameter $\Delta f=\Delta \omega / 2 \pi$ and for different mass ratios. The optimal TMD parameters are also compared with those obtained using the tuning proposed by Korenev and Reznikov, Den Hartog and that based on the APE. It should be noted that for the Korenev's and Den Hartog's tuning, the TMD locations are taken equal to those obtained for $\Delta f=40 \mathrm{~Hz}$, since both tuning cannot provide optimal placements of the TMD device. The forcing location on the plate is arbitrarily chosen as $\mathbf{r}_{F}=(0.05,0.05)^{\mathrm{T}}$.

The comparison of the optimal damping ratios presented in Table 4 with those presented in Table 3 shows that for the target frequency $\varpi_{1}$ the TMD acts as a reactive device (undamped TMD) [45] when a narrowband control is implemented, whereas it acts as a dissipative one when the target frequency $\varpi_{4}$ is controlled. In contrast, when a broadband control is performed, the TMD acts as a dissipative device for both controlled frequencies. 
To show the fact that the TMD acts as an undamped device (reactive device) if $\varpi_{1}$ is targeted, and when a narrowband control is performed ( $\Delta f=2 \mathrm{~Hz}$ ), the damping ratio $\xi_{T}$ of the TMD is set to $0 \%$ and an optimization is performed. The obtained results (for $\mu=2 \%$ ) showed that the optimal undamped TMD parameters are $f_{T}^{*}=111.001 \mathrm{~Hz}$ and $\left(x_{c}^{*}, y_{c}^{*}\right)=(0.242,0,149)$; as we can see, these results are very close to those obtained in Table 3 (when $\mu=2 \%$ and $\Delta f=2 \mathrm{~Hz}$ ). Inaddition, looking at Fig. 4 , one can see that the PSDs responses corresponding to narrowband control, for both cases (damped and undamped TMD), are roughly the same whereas they are completely differents when a broadband control ( $\Delta f=40 \mathrm{~Hz}$ ) is performed. It should be noted that similar results have been obtained for different values of the mass ratio $\mu$.

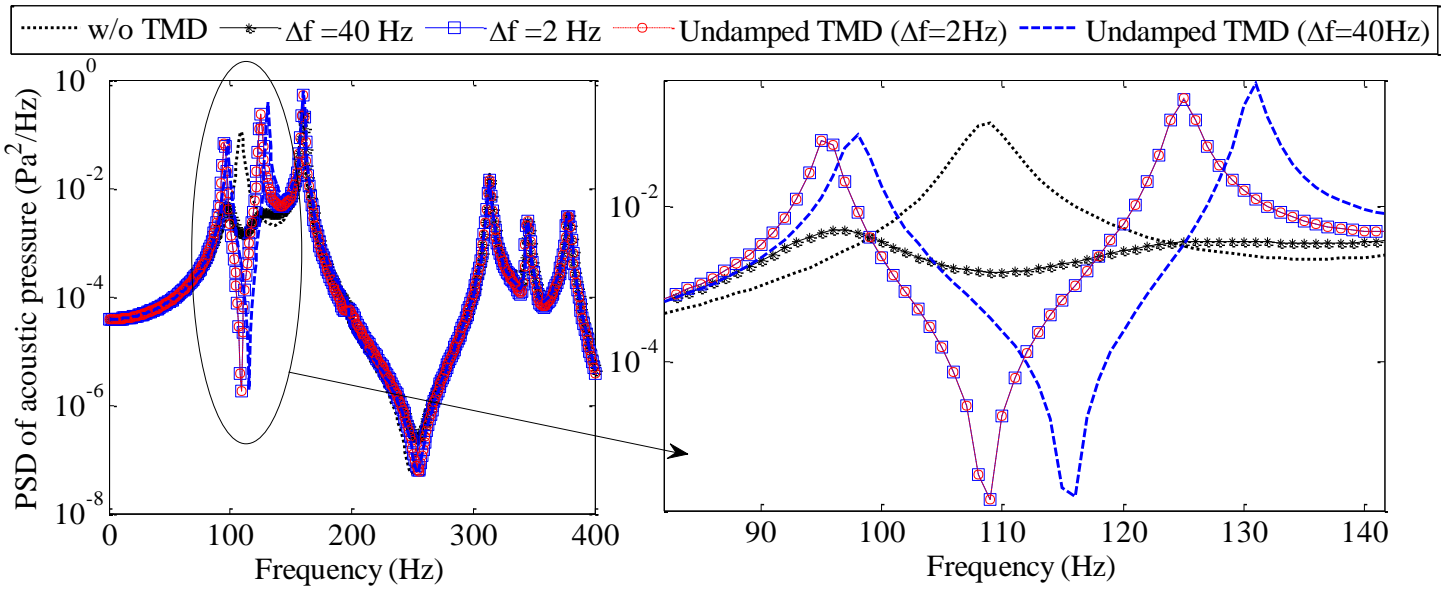

Fig. 4 PSD responses of acoustic pressure for the damped and undamped TMD, $\boldsymbol{\mu}=\mathbf{2} \%, \varpi_{1}=108.59 \mathrm{~Hz}$

Looking at the $G_{2}$ index values presented in Table 3, one can see that when a broadband control is performed ( $\Delta f=40 \mathrm{~Hz}$ ), the TMD device performs well overall the frequency range [0-400] Hz; this observation holds as the mass ratio increases. When a narrowband control is performed ( $\Delta f=2 \mathrm{~Hz}$, for instance), positive values of the $G_{2}$ index are obtained which means undesirable global performance of the TMD. The comparison of the global performance of the TMD, optimized using a broadband control, with the others, shows that it is similar to those obtained using the Korenev's and the Den Hartog's tuning. The APE strategy yields the worst global performance.

\begin{tabular}{|c|c|c|c|c|c|c|c|}
\hline \multirow[b]{2}{*}{$\mu(\%)$} & \multirow[b]{2}{*}{$\Delta f(\mathrm{~Hz})$} & \multicolumn{4}{|c|}{ TMD optimal parameters } & \multirow{2}{*}{$\sigma_{p}^{*}(\mathrm{~Pa})$} & \multirow{2}{*}{$G_{2}(\%)$} \\
\hline & & $\xi_{T}^{*}(\%)$ & $f_{T}^{*}(\mathrm{~Hz})$ & $x_{c}^{*}(\mathrm{~m})$ & $y_{c}^{*}(\mathrm{~m})$ & & \\
\hline \multirow{7}{*}{2} & 2 & 0.01 & 110.897 & 0.240 & 0.150 & 0.0027 & 15.8 \\
\hline & 10 & 0.03 & 111.106 & 0.244 & 0.150 & 0.0320 & 15.6 \\
\hline & 20 & 1.16 & 110.694 & 0.244 & 0.150 & 0.1231 & 4.0 \\
\hline & 40 & 13.45 & 110.919 & 0.253 & 0.151 & 0.3284 & -19.2 \\
\hline & Korenev & 12.97 & 110.425 & $* * *$ & $* * *$ & $* * *$ & -18.6 \\
\hline & Den Hartog & 16.66 & 102.248 & $* * *$ & $* * *$ & $* * *$ & -21.5 \\
\hline & APE & 0.96 & 108.529 & 0.232 & 0.108 & $* * *$ & 4.4 \\
\hline \multirow{2}{*}{3} & 2 & 0.01 & 111.739 & 0.235 & 0.149 & 0.0018 & 21.6 \\
\hline & 10 & 0.01 & 112.238 & 0.241 & 0.149 & 0.0209 & 23.9 \\
\hline
\end{tabular}




\begin{tabular}{ccllcccc}
\hline & $\mathbf{2 0}$ & 0.37 & 112.107 & 0.241 & 0.150 & 0.0715 & 31.3 \\
$\mathbf{4 0}$ & 14.96 & 110.612 & 0.252 & 0.151 & 0.2729 & -22.8 \\
Korenev & 15.25 & 110.425 & $* * *$ & $* * *$ & $* * *$ & -23.0 \\
Den Hartog & 20.04 & 98.595 & $* * *$ & $* * *$ & $* * *$ & -25.1 \\
$\mathbf{A P E}$ & 0.09 & 114.294 & 0.234 & 0.110 & $* * *$ & -0.9 \\
\hline $\mathbf{2}$ & 0.01 & 112.405 & 0.229 & 0.149 & 0.0014 & 26.7 \\
& $\mathbf{1 0}$ & 0.01 & 113.273 & 0.238 & 0.149 & 0.0155 & 16.8 \\
$\mathbf{4}$ & $\mathbf{2 0}$ & 0.17 & 113.304 & 0.238 & 0.149 & 0.0505 & 26.3 \\
& $\mathbf{4 0}$ & 15.06 & 110.168 & 0.248 & 0.150 & 0.2301 & -25.5 \\
& Korenev & 16.94 & 110.425 & $* * *$ & $* * *$ & $* * *$ & -27.8 \\
& Den Hartog & 22.74 & 95.197 & $* * *$ & $* * *$ & $* * *$ & -28.3 \\
& $\mathbf{A P E}$ & 0.45 & 108.554 & 0.146 & 0.160 & $* * *$ & -15.8 \\
\hline
\end{tabular}

Table 3: Optimal TMD parameters for different mass ratio and with different bandwidth control, $\varpi_{1}=108.59 \mathrm{~Hz}$

Unlike the target frequency $\varpi_{1}=108.59 \mathrm{~Hz}$, which is well separated from the other resonant frequencies, one can see that the target frequency $\varpi_{4}=344.45 \mathrm{~Hz}$ is close to neighboring resonance frequencies [ 45 , 67] therefore more than one mode will be excited by the applied primary force at the target frequency $\varpi_{4}$. This fact strongly affects the optimal frequencies of the TMD, given in Table 4, which are strongly varying when the bandwidth parameter $\Delta f$ is varying.

The comparison of the obtained results with the other optimization strategies (Korenev, Den Hartog and APE) for different bandwidth shows that the Den Hartog's optimal damping is always the highest, whereas its corresponding optimal frequency is always the smallest, except for $\varpi_{4}$ when $\mu=3$ and $4 \%$. Besides, it is observed that the optimal tuning proposed by Korenev and Reznikov is the closest to the optimal tuning proposed in the present work, especially for the optimal frequencies. This remark holds for both targeted frequencies. The optimal TMD locations $x_{c}^{*}$ and $y_{c}^{*}$ are also given in Tables 3 and 4 . The observation of Table 3 shows that optimal tuning is obtained when the TMD is placed roughly in the center of the plate which corresponds to the anti-node point of the plate mode $(1,1)$; this result is predictable because the coupled mode is strongly dominated by the plate mode. In addition, the slight alteration, from exactly the position $x=0.25$ and $y=0.15$, of the obtained optimal locations is due to the coupling effects with the cavity mode $(0,0,0)$ inducing distortion in the plate mode [27].

\begin{tabular}{|c|c|c|c|c|c|c|c|}
\hline \multirow[b]{2}{*}{$\mu(\%)$} & \multirow[b]{2}{*}{$\Delta f(\mathrm{~Hz})$} & \multicolumn{4}{|c|}{ TMD optimal parameters } & \multirow{2}{*}{$\sigma_{p}^{*}(\mathrm{~Pa})$} & \multirow{2}{*}{$G_{2}(\%)$} \\
\hline & & $\xi_{T}^{*}(\%)$ & $f_{T}^{*}(\mathrm{~Hz})$ & $x_{T M D}^{*}(\mathrm{~m})$ & $y_{T M D}^{*}(\mathrm{~m})$ & & \\
\hline \multirow{7}{*}{2} & 2 & 2.234 & 337.493 & 0.031 & 0.097 & $4.66 \times 10^{-05}$ & 4.1 \\
\hline & 10 & 2.053 & 338.687 & 0.047 & 0.067 & $1.04 \times 10^{-03}$ & 9.9 \\
\hline & 20 & 1.984 & 340.100 & 0.051 & 0.061 & $4.13 \times 10^{-03}$ & 10.9 \\
\hline & 40 & 3.588 & 334.259 & 0.055 & 0.064 & $2.29 \times 10^{-02}$ & 5.2 \\
\hline & Korenev & 7.646 & 344.266 & $* * *$ & $* * *$ & $* * *$ & 6.7 \\
\hline & Den Hartog & 9.509 & 335.966 & $* * *$ & $* * *$ & $* * *$ & 4.7 \\
\hline & APE & 3.9861 & 331.598 & 0.049 & 0.079 & $* * *$ & 47.4 \\
\hline \multirow{4}{*}{3} & 2 & 2.930 & 333.262 & 0.035 & 0.088 & $4.46 \times 10^{-05}$ & 4.7 \\
\hline & 10 & 3.617 & 331.804 & 0.041 & 0.079 & $1.12 \times 10^{-03}$ & 6.2 \\
\hline & 20 & 1.923 & 340.319 & 0.051 & 0.057 & $2.94 \times 10^{-03}$ & 6.7 \\
\hline & 40 & 3.316 & 335.858 & 0.053 & 0.061 & $1.71 \times 10^{-02}$ & 5.8 \\
\hline
\end{tabular}


Table 4: Optimal TMD parameters for different mass ratio and with different bandwidth control, $\varpi_{4}=344.45 \mathrm{~Hz}$

For the frequency $\varpi_{4}$, the optimal TMD locations given in Table 4 are close to the primary force location, especially for broadband control, and they are relatively far away when a narrowband control is performed.

The comparison of the PSD responses shown in Fig. 5 and 6 shows that a broadband control, for both targeted frequencies, yields more suitable and satisfactory performances of the TMD device. Indeed, for the target frequency $\varpi_{1}$ a reduction of $G_{1}=27.34 \mathrm{~dB}$ is achieved for a broadband control and $G_{1}=47.51 \mathrm{~dB}$ when broadband control is applied to the target frequency $\varpi_{4}$. The appearance of new undesirable peaks shown in Fig. 5, when a narrowband control is implemented, implies that such a control is not suitable and one had to use a broadband control (i.e. $\Delta f=40 \mathrm{~Hz}$ ) to effectively attenuate the resonant mode corresponding to the frequency $\varpi_{1}$.

In addition, although the reduction in the PSD responses for the target frequency $\varpi_{4}$ is more significant when a narrowband control is performed, the performance of the TMD in the vicinity of the off-target frequencies is less suitable. Therefore a broadband control is more appropriate.

The results of the global performance index $G_{2}$ stated in Table 4 show that the RMSAP has increased when the TMD is used; these results are predictable considering the PSD responses in Fig. 6 where undesirable increases of the PSD responses are observed in the vicinity of the off-target frequencies. The obtained results hold for all optimization strategies; also here the APE strategy yields the worst performance. The Korenev's and Den Hartog's tuning provide global performance similar to that of the proposed strategy.

In Fig. 7 and 8, the performance of the TMD device when it's tuned using the proposed strategy is compared, with its performance if it is tuned using the tuning proposed by Korenev and Reznikov, Den Hartog and the APE strategy; for these last strategies the mass ratio is set to $\mu=4 \%$. The bandwidth parameter is set to $\Delta f=40 \mathrm{~Hz}$ and three values of the mass ratio are considered.

The results show that the best performance is achieved for the largest mass ratio ( $\mu=4 \%$ ), for which the Korenev's tuning is the closest to the proposed tuning, especially for the well separated target natural frequency $\varpi_{1}$. 
For the target frequency $\varpi_{1}$ (Fig. 7), a reduction of $G_{1}=30.96 \mathrm{~dB}$ in the PSD response can be achieved when the proposed strategy is applied, whereas two undesirable peaks have shown up (in the vicinity of the target frequency) when the APE strategy is used.

Besides, although the APE strategy yields good performance (for the target frequency $\varpi_{4}$ ) with a reduction of $G_{1}=99.70 \mathrm{~dB}$ in the very close vicinity of the targeted frequency, the appearance of a new peak in the vicinity of the off-target frequency $312 \mathrm{~Hz}$, diminishes the global performance of the APE strategy. Using the proposed optimization strategy a reduction of $G_{1}=56.1 \mathrm{~dB}$ can be achieved (as shown in Fig. 8) and no significant effects have been observed on the off-target frequencies.

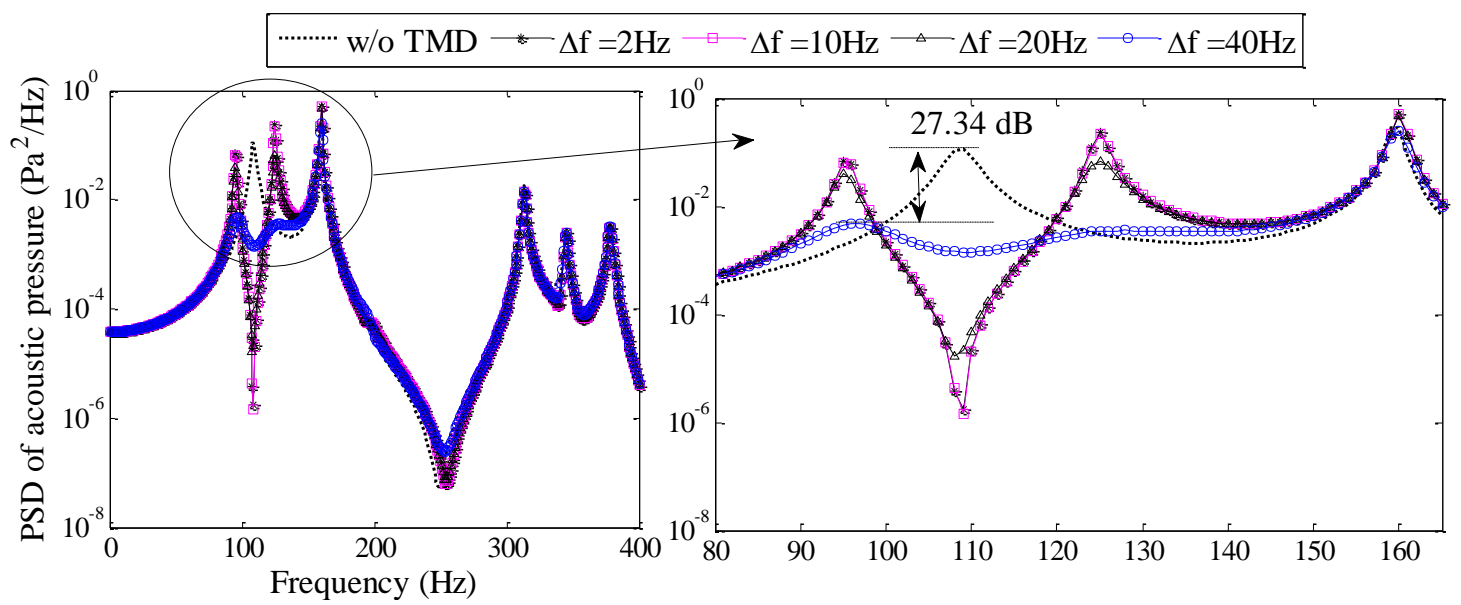

Fig. 5: PSD responses of acoustic pressure for narrowband and broadband control, $\boldsymbol{\mu}=\mathbf{2 \%}, \varpi_{1}=108.59 \mathrm{~Hz}$

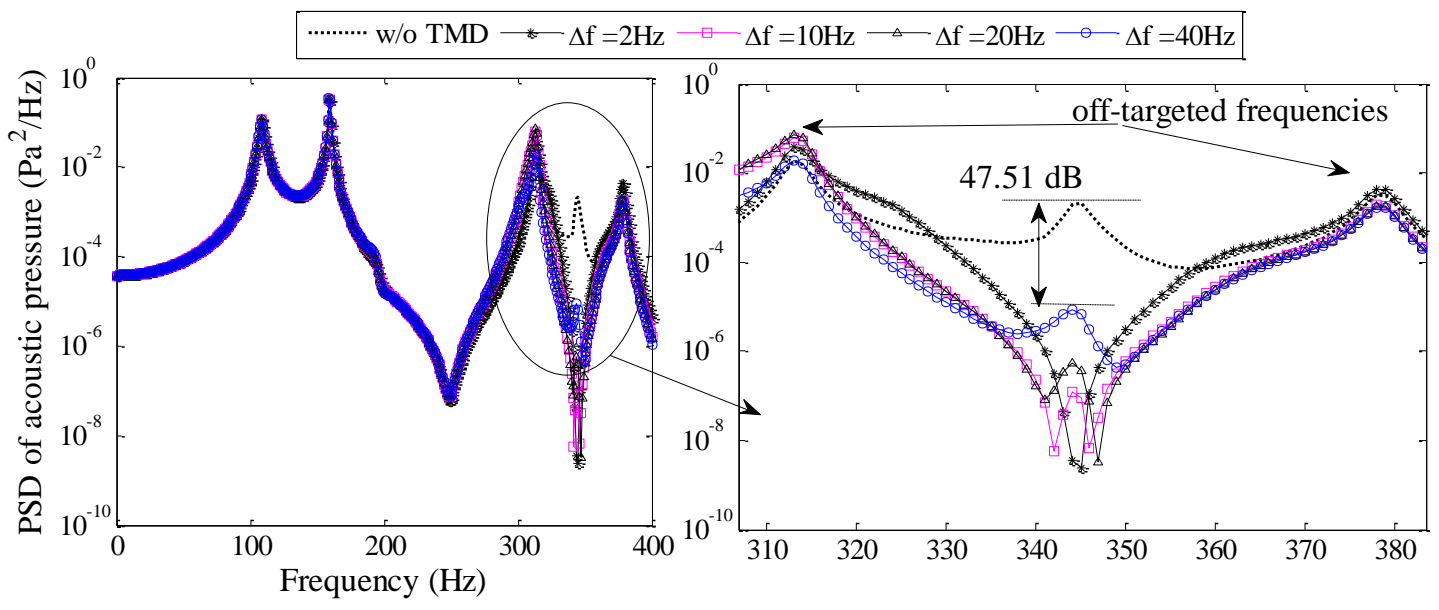

Fig. 6: PSD responses of acoustic pressure for narrowband and broadband control, $\mu=2 \%, \varpi_{4}=343.8 \mathrm{~Hz}$ 


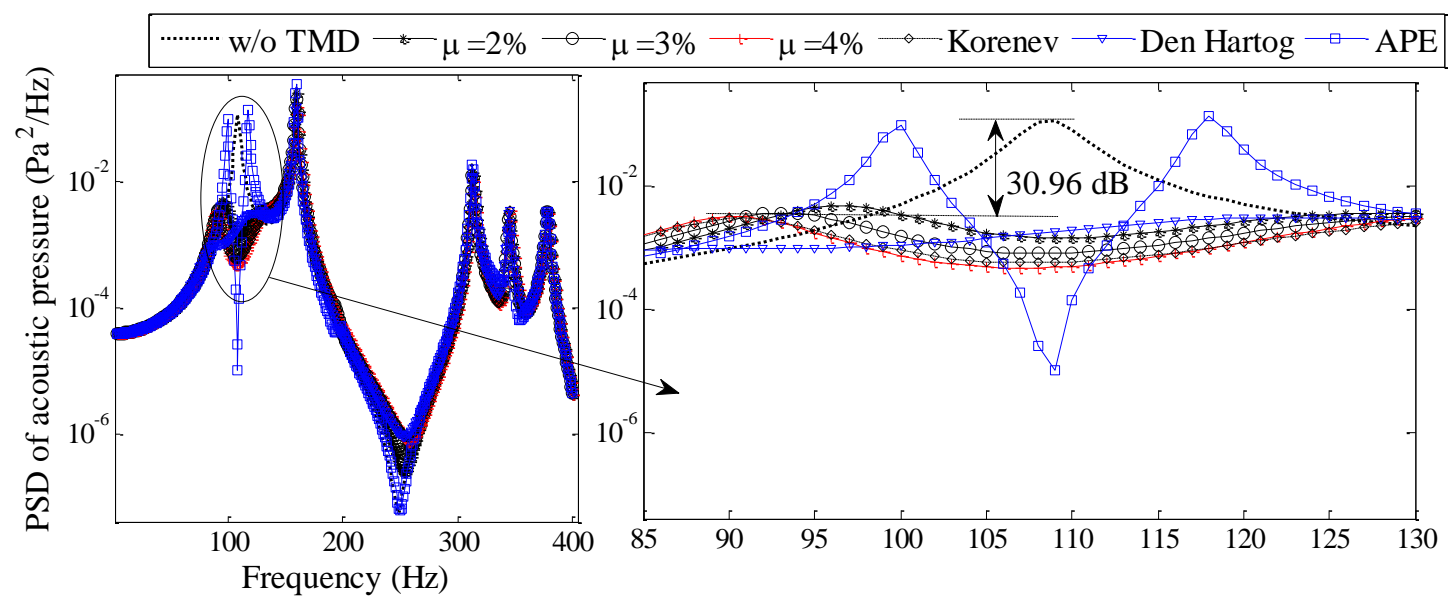

Fig. 7: PSD responses of acoustic pressure for a broadband control $(\Delta \mathrm{f}=40 \mathrm{~Hz})$ and different mass ratios, $\varpi_{1}=108.59 \mathrm{~Hz}$

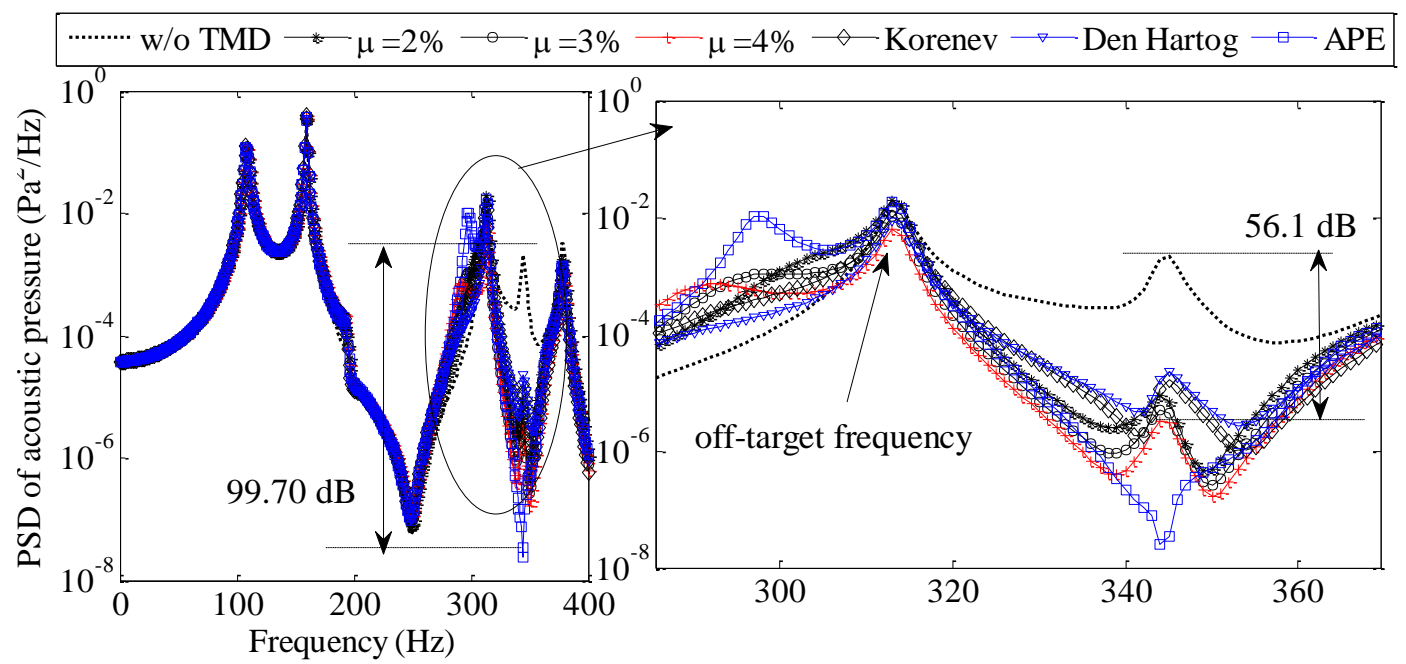

Fig. 8: PSD responses for a broadband control $(\boldsymbol{\Delta} \mathbf{f}=\mathbf{4 0 H z})$ and different mass ratios;, $\varpi_{4}=343.8 \mathrm{~Hz}$

\subsubsection{Control of the modes dominated by cavity modes}

Tables 5-7 give the optimal TMD parameters when the modes corresponding to the frequencies $\varpi_{2}, \varpi_{3}$ and $\varpi_{5}$ are controlled. These results are obtained for different values of the bandwidth parameter and for different values of the mass ratio. The obtained results are also compared with those established when the strategy based on APE is applied. The examination of the obtained optimal damping ratios (presented in Tables 5-7) shows that the TMD acts as a reactive device for both kinds of control (narrowband and broadband). Indeed, one can see that the optimal damping ratios are very small (less than 3\%) and for some values of $\Delta f$, they are equal to $0.01 \%$ which means that the device acts as an undamped TMD. This result is coherent with other results found in the literature [3] where the greatest acoustic pressure reduction occurs when the TMD device acts as a highly reactive device and the effect of the dissipation process [45] is reduced to zero. For these coupled modes dominated by cavity modes, the TMD device acts like as a neutralizer $[13,16]$. 


\begin{tabular}{|c|c|c|c|c|c|c|c|}
\hline \multirow[b]{2}{*}{$\mu(\%)$} & \multirow[b]{2}{*}{$\Delta f(\mathrm{~Hz})$} & \multicolumn{4}{|c|}{ TMD optimal parameters } & \multirow{2}{*}{$\sigma_{p}^{*} \times 10^{-4}(\mathrm{~Pa})$} & \multirow{2}{*}{$G_{2}(\%)$} \\
\hline & & $\xi_{T}^{*}(\%)$ & $f_{T}^{*}(\mathrm{~Hz})$ & $x_{T M D}^{*}(\mathrm{~m})$ & $y_{T M D}^{*}(\mathrm{~m})$ & & \\
\hline \multirow{5}{*}{2} & 2 & 0.693 & 155.554 & 0.192 & 0.147 & 77.094 & -33.8 \\
\hline & 10 & 0.676 & 153.279 & 0.141 & 0.132 & 725.867 & -21.5 \\
\hline & 20 & 0.010 & 175.472 & 0.261 & 0.156 & 1249.856 & -8.3 \\
\hline & 40 & 0.010 & 184.877 & 0.275 & 0.156 & 2103.574 & -19.7 \\
\hline & APE & 0.6708 & 155.794 & 0.176 & 0.172 & $* * *$ & -8.6 \\
\hline \multirow{5}{*}{3} & 2 & 1.043 & 152.277 & 0.184 & 0.146 & 38.186 & -38.0 \\
\hline & $\mathbf{1 0}$ & 1.026 & 150.374 & 0.151 & 0.136 & 365.339 & -30.1 \\
\hline & 20 & 0.010 & 175.424 & 0.247 & 0.157 & 883.536 & -26.4 \\
\hline & 40 & 0.010 & 183.097 & 0.256 & 0.157 & 1408.895 & -19.7 \\
\hline & APE & 0.519 & 163.519 & 0.222 & 0.157 & $* * *$ & -21.9 \\
\hline \multirow{5}{*}{4} & 2 & 1.341 & 149.323 & 0.180 & 0.145 & 19.371 & -41.1 \\
\hline & 10 & 1.355 & 147.578 & 0.158 & 0.139 & 189.293 & -36.5 \\
\hline & 20 & 1.185 & 146.967 & 0.146 & 0.132 & 503.278 & -32.0 \\
\hline & 40 & 0.010 & 178.974 & 0.243 & 0.158 & 1028.770 & -39.9 \\
\hline & APE & 3.324 & 166.133 & 0.217 & 0.182 & $* * *$ & -25.4 \\
\hline
\end{tabular}

Table 5: Optimal TMD parameters for different mass ratio and with different bandwidth control, $\varpi_{2}=159.52 \mathrm{~Hz}$

The optimal TMD locations given in Tables 5 (control of the target frequency $\varpi_{2}$ ) show that they are roughly in the vicinity of the anti-node of the plate mode $(1,1)$. The fact that the TMD locations are not exactly at the anti-node (for narrowband control) is due to the coupling effects with the cavity mode $(0,0,1)$ and with the plate mode $(2,1)$ which has an anti-node at coordinate $(0.125,0.15)$. The effect of the coupling with the plate mode $(2,1)$ is gradually increasing as the mass ratio $\mu$ increases. Indeed, the TMD location is moving towards the anti-node of the mode $(2,1)$. For the broadband control, one can see that the TMD locations are around the anti-node of the plate mode $(1,1)$.

The optimal TMD locations, when the frequency $\varpi_{3}$ is controlled, are given in Table 6 where it has been observed that for narrowband and broadband control, the optimal locations were in the vicinity of the antinode point of the plate mode $(3,1)$.

\begin{tabular}{|c|c|c|c|c|c|c|c|}
\hline \multirow[b]{2}{*}{$\mu(\%)$} & \multirow[b]{2}{*}{$\Delta f(\mathrm{~Hz})$} & \multicolumn{4}{|c|}{ TMD optimal parameters } & \multirow{2}{*}{$\sigma_{p}^{*} \times 10^{-4}(\mathrm{~Pa})$} & \multirow[b]{2}{*}{$G_{2}(\%)$} \\
\hline & & $\xi_{T}^{*}(\%)$ & $f_{T}^{*}(\mathrm{~Hz})$ & $x_{T M D}^{*}(\mathrm{~m})$ & $y_{T M D}^{*}(\mathrm{~m})$ & & \\
\hline \multirow{5}{*}{2} & 2 & 0.022 & 321.830 & 0.417 & 0.173 & 0.789 & 28.3 \\
\hline & 10 & 0.090 & 320.331 & 0.426 & 0.168 & 21.580 & 8.1 \\
\hline & 20 & 0.186 & 318.665 & 0.431 & 0.162 & 74.52473 & 8.2 \\
\hline & 40 & 0.366 & 307.183 & 0.066 & 0.093 & 254.940 & 8.9 \\
\hline & APE & 0.159 & 309.059 & 0.046 & 0.081 & $* * *$ & 0.3 \\
\hline \multirow{5}{*}{3} & 2 & 0.010 & 325.745 & 0.446 & 0.163 & 1.215 & 9.6 \\
\hline & 10 & 0.010 & 325.621 & 0.446 & 0.164 & 19.427 & 8.8 \\
\hline & 20 & 0.010 & 325.750 & 0.445 & 0.166 & 65.375 & 7.9 \\
\hline & 40 & 0.464 & 305.144 & 0.062 & 0.093 & 165.545 & 14.1 \\
\hline & APE & 0.194 & 307.598 & 0.043 & 0.082 & $* * *$ & 5.6 \\
\hline \multirow{4}{*}{4} & 2 & 0.010 & 328.523 & 0.456 & 0.158 & 1.382 & 9.5 \\
\hline & 10 & 0.010 & 328.386 & 0.456 & 0.159 & 19.187 & 8.6 \\
\hline & 20 & 0.010 & 328.903 & 0.454 & 0.163 & 63.263 & 7.7 \\
\hline & 40 & 0.551 & 302.808 & 0.059 & 0.092 & 125.255 & 19.2 \\
\hline
\end{tabular}


APE

Table 6: Optimal TMD parameters for different mass ratio and with different bandwidth control, $\varpi_{3}=313.20 \mathrm{~Hz}$

The optimal TMD locations corresponding to the control of the frequency $\varpi_{5}$ are also presented in Table 7. The results show that they are in the vicinity of the forcing location and they are relatively far away when a broadband control is performed.

\begin{tabular}{|c|c|c|c|c|c|c|c|}
\hline \multirow[b]{2}{*}{$\mu(\%)$} & \multirow[b]{2}{*}{$\Delta f(\mathrm{~Hz})$} & \multicolumn{4}{|c|}{ TMD optimal parameters } & \multirow{2}{*}{$\sigma_{p}^{*} \times 10^{-4}(\mathrm{~Pa})$} & \multirow[b]{2}{*}{$G_{2}(\%)$} \\
\hline & & $\xi_{T}^{*}(\%)$ & $f_{T}^{*}(\mathrm{~Hz})$ & $x_{T M D}^{*}(\mathrm{~m})$ & $y_{T M D}^{*}(\mathrm{~m})$ & & \\
\hline \multirow{5}{*}{2} & 2 & 2.163 & 365.795 & 0.064 & 0.071 & 0.242 & 2.8 \\
\hline & 10 & 2.173 & 366.019 & 0.067 & 0.067 & 7,077 & 14.6 \\
\hline & 20 & 2.122 & 378.299 & 0.048 & 0.104 & 27.392 & 25.1 \\
\hline & 40 & 1.915 & 366.677 & 0.062 & 0.070 & 63.377 & 27.5 \\
\hline & APE & 0.854 & 371.300 & 0.080 & 0.046 & $* * *$ & 2.7 \\
\hline \multirow{5}{*}{3} & 2 & 2.143 & 363.097 & 0.057 & 0.071 & 0.187 & 20.7 \\
\hline & 10 & 2.310 & 374.864 & 0.092 & 0.035 & 12.680 & 13.9 \\
\hline & 20 & 2.128 & 377.796 & 0.041 & 0.104 & 24.377 & 17.5 \\
\hline & 40 & 1.744 & 416.471 & 0.040 & 0.134 & 63.690 & 15.3 \\
\hline & APE & 2.624 & 360.625 & 0.060 & 0.072 & $* * *$ & 13.9 \\
\hline \multirow{5}{*}{4} & 2 & 2.216 & 366.139 & 0.070 & 0.049 & 1.122 & 22.2 \\
\hline & 10 & 2.177 & 363.564 & 0.064 & 0.056 & 5.292 & 13.9 \\
\hline & 20 & 2.134 & 362.738 & 0.061 & 0.060 & 14.528 & 19.8 \\
\hline & 40 & 1.923 & 363.275 & 0.057 & 0.064 & 42.348 & 12.1 \\
\hline & APE & 0.990 & 381.270 & 0.028 & 0.111 & $* * *$ & 15.1 \\
\hline
\end{tabular}

Table 7: Optimal TMD parameters for different mass ratio and with different bandwidth control, $\varpi_{5}=378.61 \mathrm{~Hz}$

The results of the $G_{2}$ index stated in Table 5 show that good global performance of the TMD device is achieved when a narrowband control is performed ( $\Delta f=2 \mathrm{~Hz}$ ). Indeed, for $\mu=4 \%$, the obtained reduction in the RMSAP is $G_{2}=-41.1 \%$ while it is $-25.4 \%$ when the APE strategy is applied.

In Tables 6 and 7 the obtained values of the $G_{2}$ index are also shown for different bandwidth and for different mass ratio. The results show that the best global performances of the TMD are achieved when the APE strategy is applied.

The pressure PSD responses of the vibro-acoustic system, for different bandwidth parameters, are shown in Fig. 9-11. The inspection of the obtained curves shows that when the frequencies $\varpi_{2}$ and $\varpi_{5}$ are controlled, satisfactory performance can be achieved when a narrowband control ( $\Delta f=2 \mathrm{~Hz}$ ) is performed for which reductions of $G_{1}=17.64 \mathrm{~dB}$ and $172.91 \mathrm{~dB}$ are achieved, respectively. In contrast, when the frequency $\varpi_{3}$ is controlled, a broadband control $\left(\Delta f=40 \mathrm{~Hz}\right.$ ) performs well and a reduction of $G_{1}=58.41$ $\mathrm{dB}$ is obtained; for the narrowband control, undesirable effects occur in the vicinity of the off-target frequencies (as shown in Fig. 10) and an increase of $23.86 \mathrm{~dB}\left(G_{1}=-23.86 \mathrm{~dB}\right)$ has been recorded in the peak's magnitude of the off-target frequency $344.45 \mathrm{~Hz}$. These magnitude increases in the vicinity of the off-target frequencies are the main cause of the high values of the $G_{2}$ index stated in Table 6. 

performed $\left(\Delta f=40 \mathrm{~Hz}\right.$ ) for the target frequency $\varpi_{2}$, the appearance of a high magnitude peak in the vicinity of the frequency $185 \mathrm{~Hz}$, is an undesirable result. The appearance of a new peak with relatively high magnitude can be explained by the very small optimal damping ratio stated in Table 5.

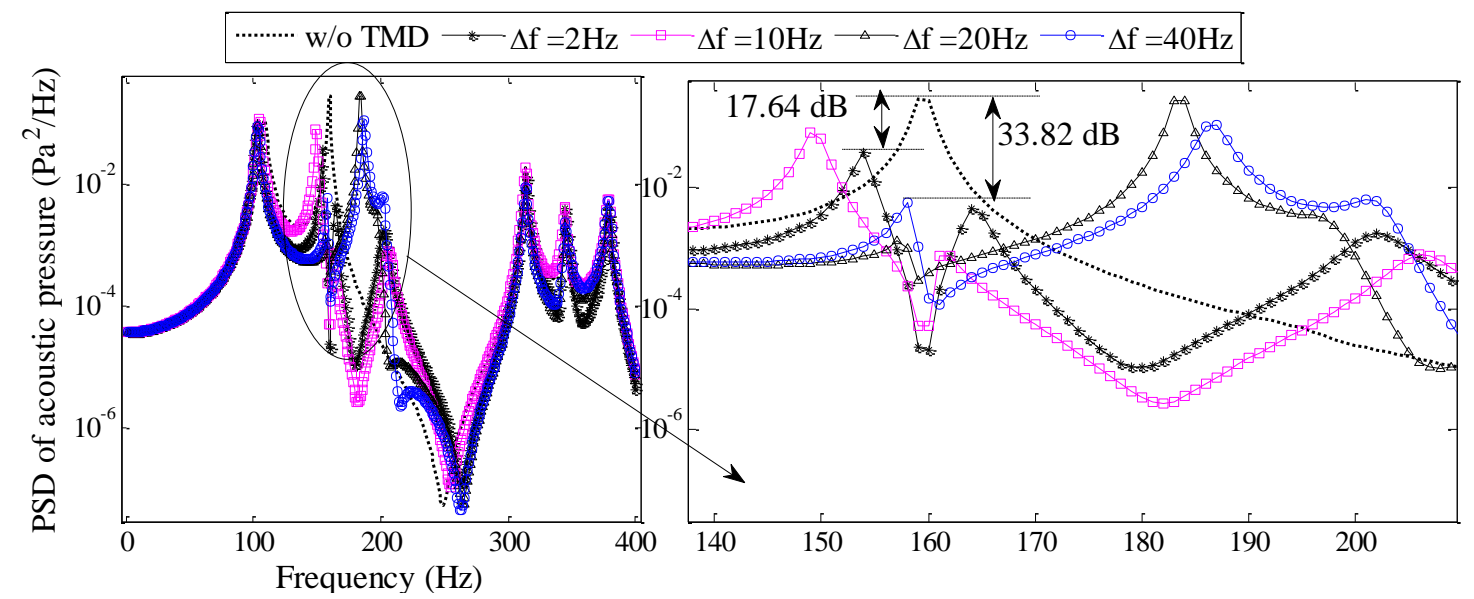

Fig. 9: PSD of acoustic pressure for narrowband and broadband control, $\mu=2 \%, \varpi_{2}=159.52 \mathrm{~Hz}$

The comparison of the performance of the proposed strategy with that based on APE $(\mu=4 \%)$ is shown in Fig. 12-14, considering different values of the mass ratio. The results show that in the close vicinity of the target frequencies, the proposed strategy performs better than that based on APE. For instance, Fig. 12 shows that a reduction of $G_{1}=31.61 \mathrm{~dB}$, in the pressure PSD, can be achieved with the proposed optimization strategy when the mass ratio is set to $\mu=4 \%$, whereas only $16.36 \mathrm{~dB}$ are achieved when the strategy based on APE is applied. Nevertheless, Looking at the effects on the off-target frequencies when $\varpi_{3}$ and $\varpi_{5}$ are controlled, the tuning based on APE strategy yields better global performance. These undesirable effects on the off-target frequencies are the main cause of the obtained values of the $G_{2}$ index (stated in Tables 6 and 7) where the better global performance of the TMD is achieved when the APE strategy is applied.

Even though the APE strategy yields good performance (compared to the one proposed) above the target frequencies corresponding to some modes dominated by cavity mode (Fig. 12 and 13), the proposed optimization strategy, using a suitable bandwidth $\Delta f$, remains superior to the APE strategy. Indeed, considering the criteria established in sub-section 4.2, we can see that significant attenuations along with relatively low magnitudes of the new peaks (in the PSD responses) can be obtained in the close vicinity of the target frequencies. The undesirable effects on the off-target frequencies (for example, in the vicinity of the frequency $380 \mathrm{~Hz}$, in Fig. 13) observed when the proposed strategy is applied are, in our opinion, acceptable. 


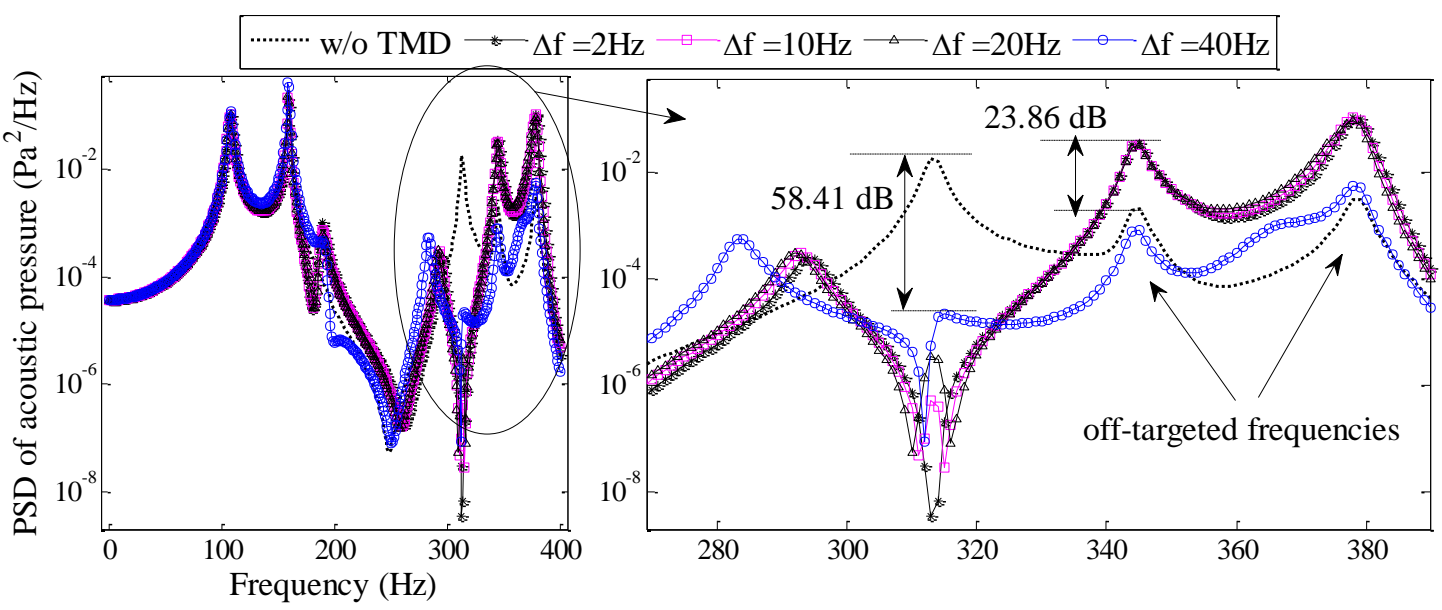

Fig. 10: PSD responses of acoustic pressure for different bandwidth parameter $\Delta \mathrm{f}, \mu=2 \%, \varpi_{3}=313.20 \mathrm{~Hz}$

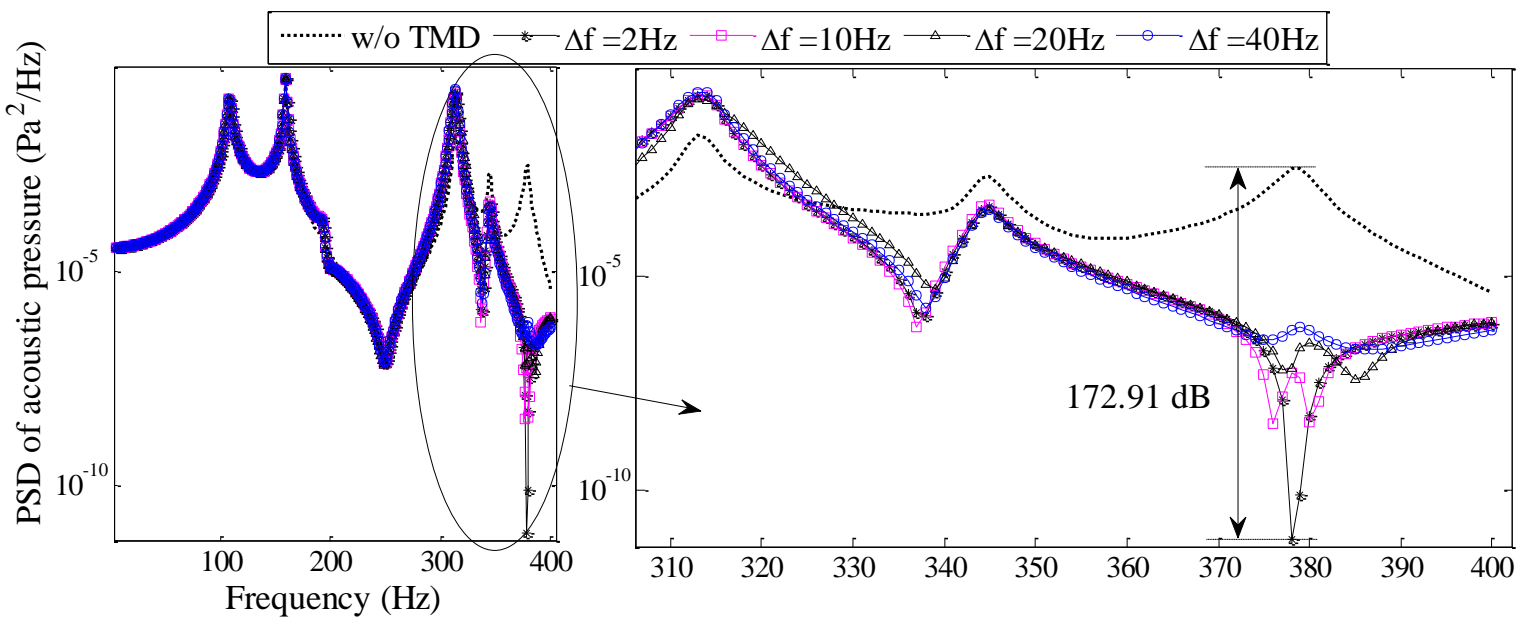

Fig. 11: PSD of acoustic responses for different values $\Delta \mathbf{f}, \mu=2 \%, \varpi_{5}=378.61 \mathrm{~Hz}$ 


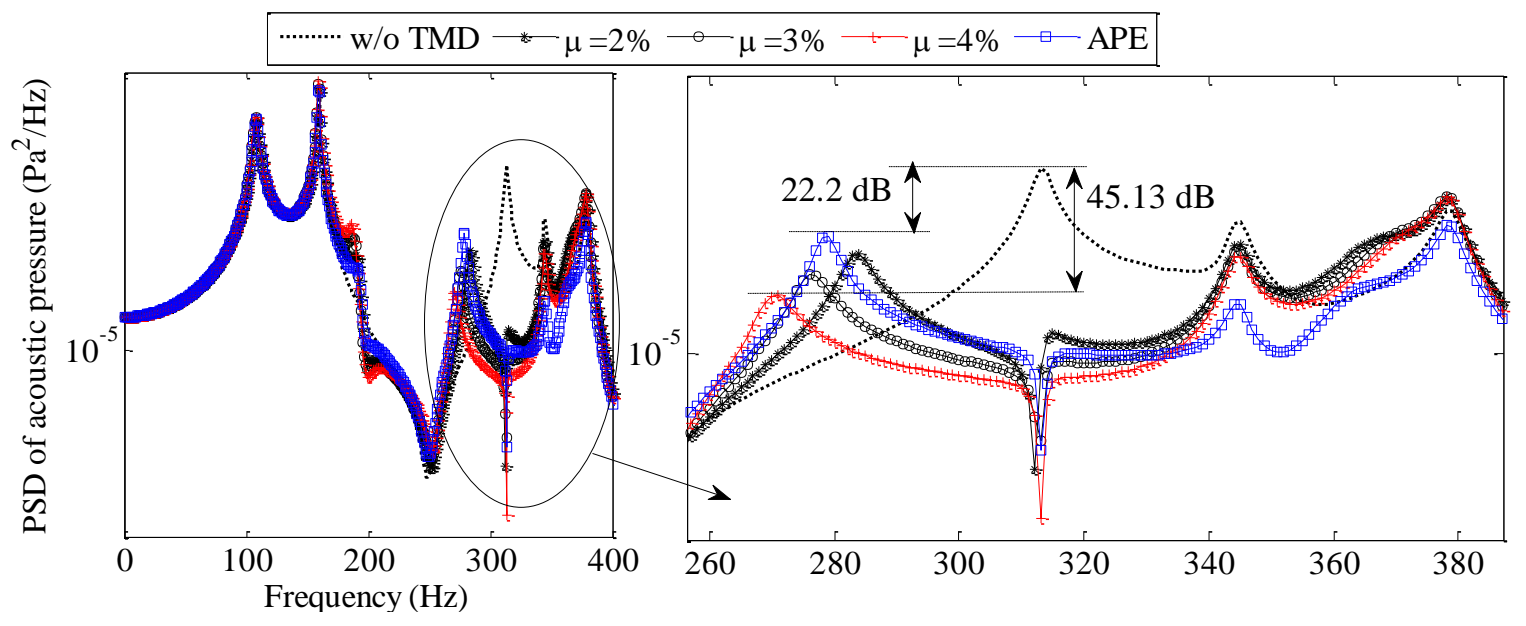

Fig. 13: PSD of acoustic responses for different mass ratio $\mu, \Delta \mathbf{f}=\mathbf{4 0 H z} ; \varpi_{3}=313.20 \mathrm{~Hz}$

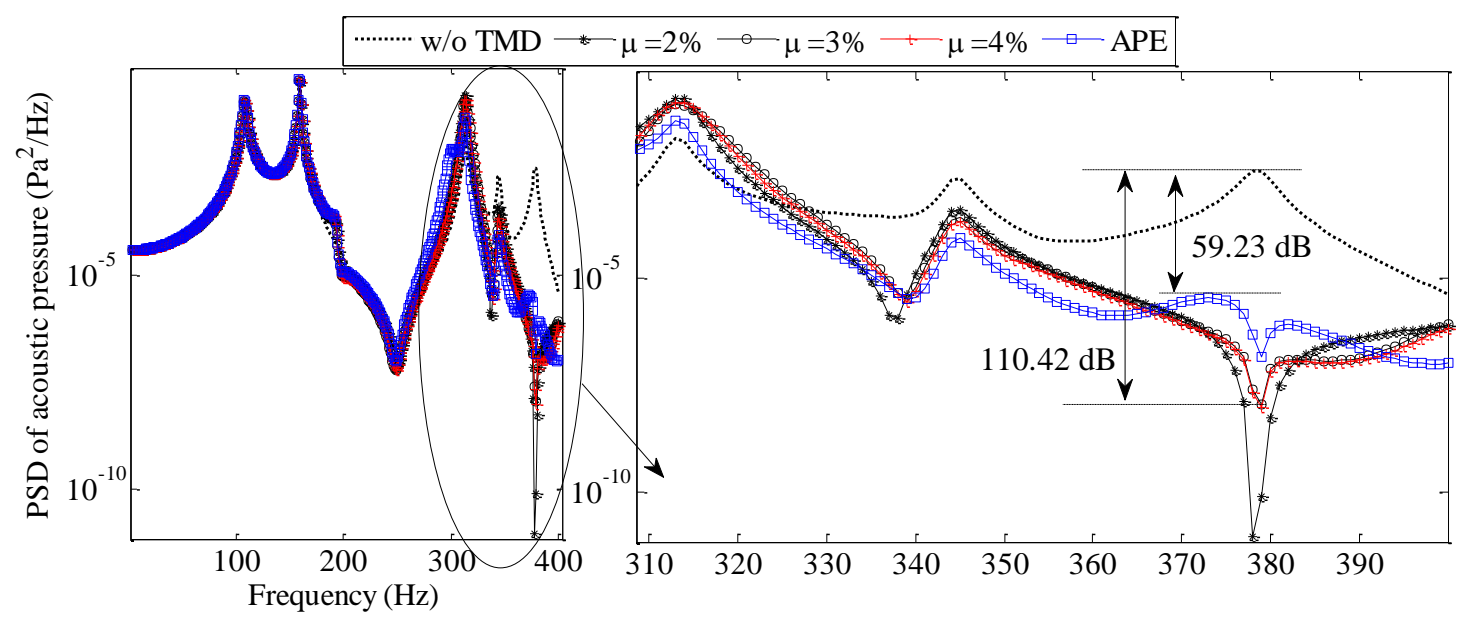

Fig. 14: PSD responses of acoustic pressure for narrowband control $(\Delta \mathrm{f}=\mathbf{2 H z})$ for different values of the mass ratio; $\varpi_{5}=378.61 \mathrm{~Hz}$ 

to address multimodal control of interior sound using multiple TMDs. The force and microphone locations are maintained at $\mathbf{r}_{F}=(0.05,0.05)^{\mathrm{T}}$ and $\mathbf{r}_{a}=(0.35,0.1,-0.875)^{\mathrm{T}}$, respectively.

\subsubsection{Control of two coupled resonant modes}

To illustrate the ability of the proposed optimization strategy to deal with multimodal control, the first two resonant modes at $\varpi_{1}=108.59 \mathrm{~Hz}$ and $\varpi_{2}=159.52 \mathrm{~Hz}$ are chosen to be controlled using two TMDs. Therefore, ten parameters need to be optimized (i.e. five parameters for each of the TMD devices). The first resonant mode is controlled by a plate mode, consequently, taking into account the results obtained in the previous section, a broadband control should be used $(\Delta f=40 \mathrm{~Hz})$ for the evaluation of the first objective function. In addition, a narrowband control corresponding to $\Delta f=2 \mathrm{~Hz}$ is used to evaluate the objective function related to the second resonant mode, controlled by the cavity.

The TMD parameters optimization has been performed using the controlled elitist GA implemented in Matlab and the initial population size is taken equal to 400 . The other algorithm parameters are taken as those set by default in Matlab.

Fig. 15 shows the Pareto front, composed by 140 solutions, obtained after 1000 generations. In order to help the decision-making, the SOM are presented in Fig. 16 where the optimized TMD parameters and both objective functions have been used for the training of the SOM. The results in Fig. 15 show the conflicting aspect of the obtained optimal solutions, where for very small values of the objective function one (mode $\varpi_{1}=108.59 \mathrm{~Hz}$ ), the objective function two has high values and vice versa. In addition, one can see that the obtained solutions are evenly distributed except for the region where the objective function one has values between roughly 0.2 and 0.24 , and the region near to 0.17 where a slight gap can be observed.

The observations made in Fig. 15 can also be seen in Fig. 16 (a) depicting the Unified distance matrix, (Umatrix) $[60,61]$ where the gaps are represented by red hexagonal lattices.

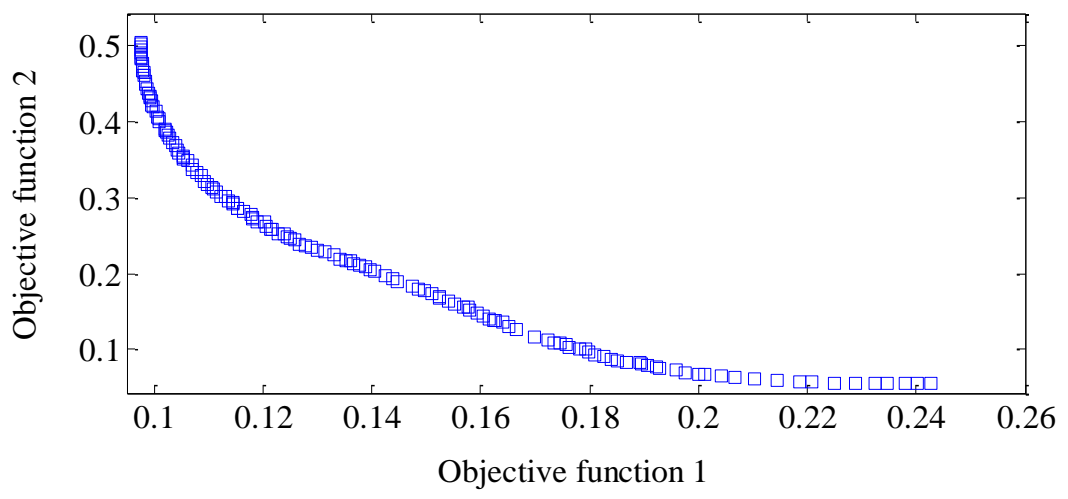

Fig. 15: Pareto front (two objective functions) 


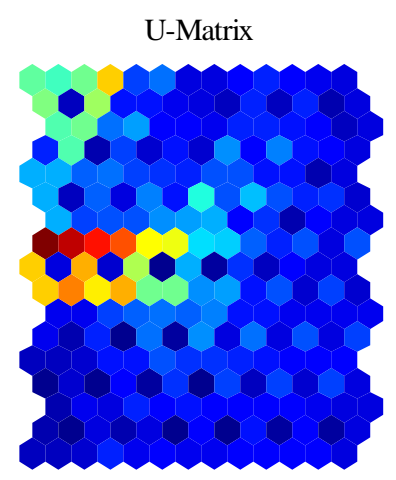

(a)

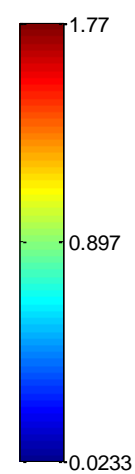
Fig. 17.

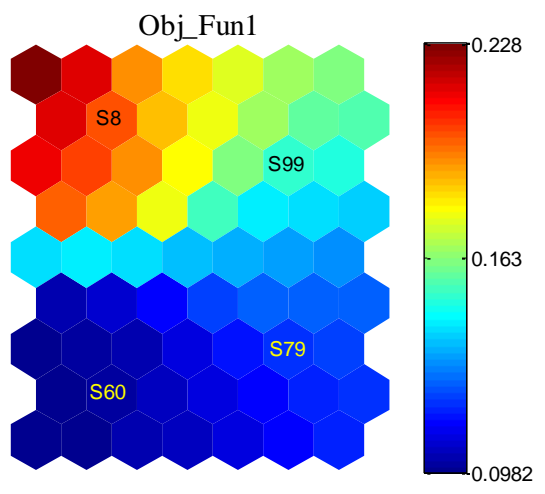

(b)

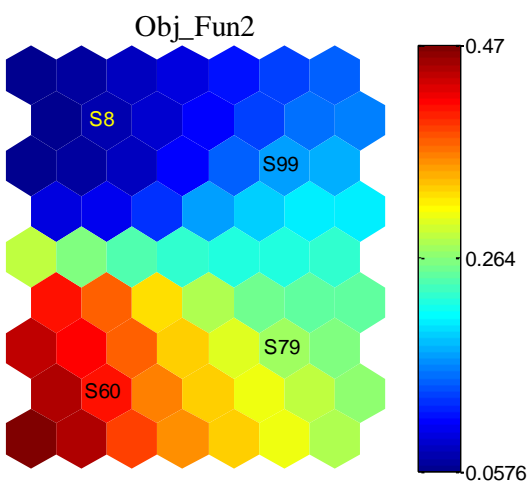

(c)

Fig. 16: SOM; (a) U-matrix map, (b) Objective function one component map; (c) Objective function two component map

The conflicting aspect of the obtained optimal solutions is also highlighted in Fig. 16 (b) and (c). Indeed, one can see that for small values of the objective function one (lower left corner in Fig. 16 (b)), the values of the objective function two are, on the contrary, at their highest values (lower left corner in Fig. 16 (c)). Also, the examination of Fig. 16 (b) and (c) demonstrate that one can define four clusters of optimal solutions represented by the four solutions S8, S60, S79 and S99. The optimal TMD parameters corresponding to these solutions are stated in Table 8.

The results given in Table 8 demonstrate that the optimal mass ratios of the second TMD (TMD 2) are always greater than those of the first TMD, for all the four solutions. Besides, one can also see that the optimal natural frequencies of the first TMD is roughly constant for all four solutions, whereas they are different for the second TMD. The values of the $G_{2}$ index stated in Table 8 are obtained when both TMD devices are mounted at the plate. The values of the global performance index $G_{2}$ show that the best performance is obtained when the solution S8 is used.

When a designer has to make a particular choice of TMD parameters, he has to make it among the solutions belonging to one of these clusters and in order to help the decision-making, the performances of the selected set of solutions (S8, S60, S79 and S99) are investigated using the PSD responses plotted in

\begin{tabular}{|c|c|c|c|c|c|c|c|c|}
\hline \multirow[b]{2}{*}{ TMDs } & \multirow[b]{2}{*}{$\begin{array}{l}\text { Optimal } \\
\text { solution }\end{array}$} & \multicolumn{5}{|c|}{ TMDs optimal parameters } & \multirow[b]{2}{*}{$\sigma_{p i}^{*}(\mathrm{~Pa})$} & \multirow[b]{2}{*}{$G_{2}(\%)$} \\
\hline & & $\xi_{T}^{*}(\%)$ & $f_{T}^{*}(\mathrm{~Hz})$ & $x_{T M D}^{*}(\mathrm{~m})$ & $y_{T M D}^{*}(\mathrm{~m})$ & $\mu_{T}^{*}(\%)$ & & \\
\hline \multirow{4}{*}{ TMD 1} & S8 & 8.451 & 99.279 & 0.185 & 0.140 & 3.038 & 0.201 & -62.7 \\
\hline & S60 & 6.505 & 99.483 & 0.209 & 0.147 & 3.184 & 0.100 & 0.9 \\
\hline & S79 & 7.257 & 99.417 & 0.202 & 0.146 & 3.092 & 0.112 & -26.4 \\
\hline & S99 & 8.333 & 99.370 & 0.193 & 0.142 & 3.061 & 0.152 & -50.2 \\
\hline \multirow{4}{*}{ TMD 2} & S8 & 2.065 & 142.276 & 0.175 & 0.138 & 3.991 & 0.067 & \\
\hline & S60 & 3.785 & 130.691 & 0.257 & 0.152 & 3.834 & 0.413 & \\
\hline & S79 & 4.024 & 130.778 & 0.229 & 0.151 & 3.937 & 0.302 & \\
\hline & S99 & 4.221 & 138.563 & 0.208 & 0.146 & 3.963 & 0.169 & \\
\hline
\end{tabular}

Table 8: Optimal parameters of the two TMDs for different optimal solutions 


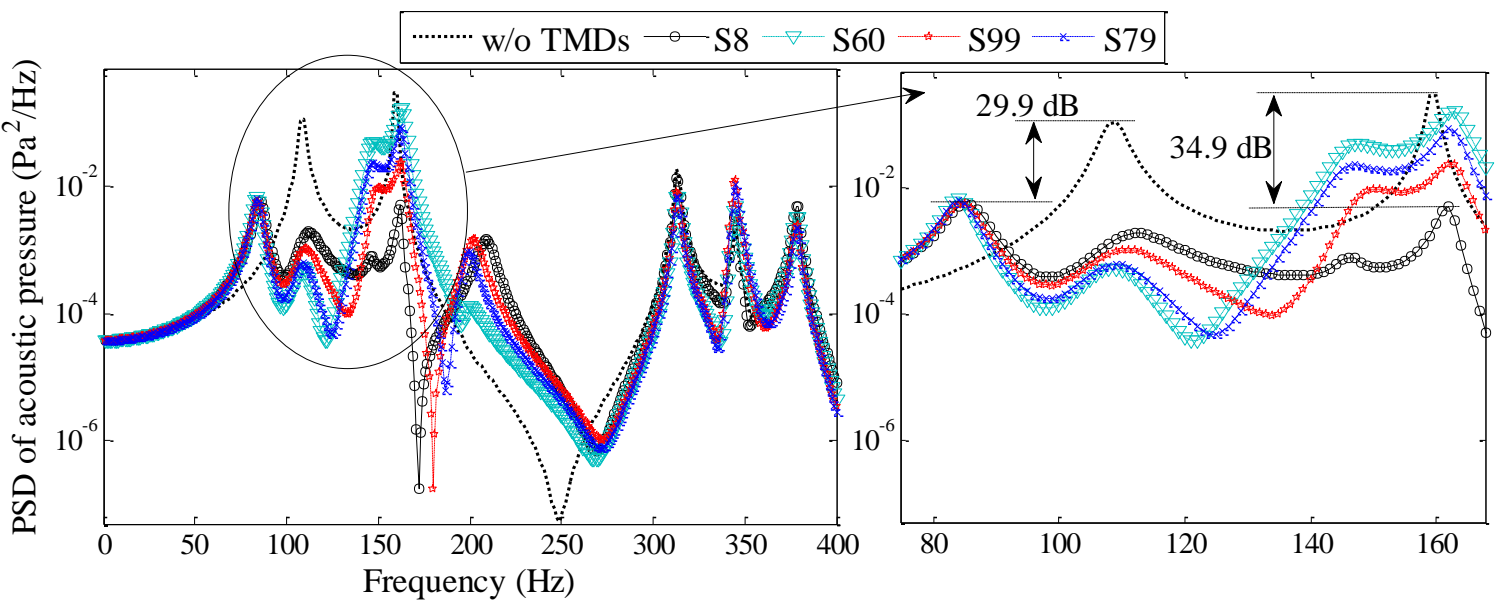

Fig. 17: PSD responses of the four selected optimal solutions (sound control using two TMDs)

The results in Fig. 17 show that the "best" performance is obtained for the solutions belonging to the first cluster containing the representative solution S8. This result is coherent with the obtained $G_{2}$ index corresponding to the solution S8 for which a reduction of $62.7 \%\left(G_{1}=-62.7 \%\right)$ is achieved.

Besides, one can see (in Fig. 17) that a reduction of $G_{1}=29.9 \mathrm{~dB}$ is achieved for the first mode and $G_{1}=34.9 \mathrm{~dB}$ for the second mode; for the off-target modes, no significant changes of the PSD responses are observed, except in the vicinity of the frequency of $200 \mathrm{~Hz}$ where a new peak appears due to the coupling effect between the TMDs and the plate-cavity mode corresponding to $196.52 \mathrm{~Hz}$.

The obtained result, for the "best" solution, is relatively unexpected since generally speaking one had to make a certain compromise and select a solution among the clusters represented by solutions S79 or S99 and not a solution belonging to a cluster where a given objective function is very high and the other is too low.

This result can be explained looking at the component maps of the optimized damping ratios shown in Fig. 18. Indeed, the results in Fig. 18 (b) show that small values of the damping ratio of the second TMD, tuned to the second resonant mode, occur only at the higher left corner of the map corresponding to the cluster containing the optimal solution S8.

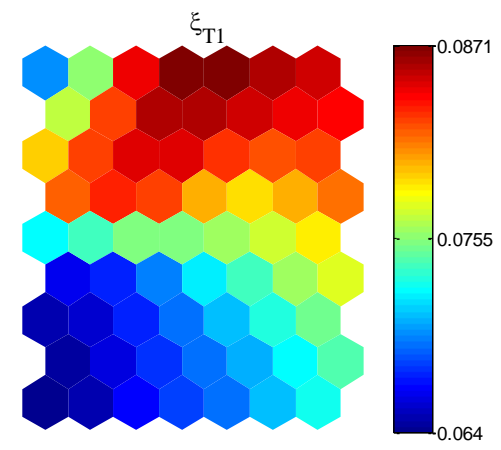

(a)

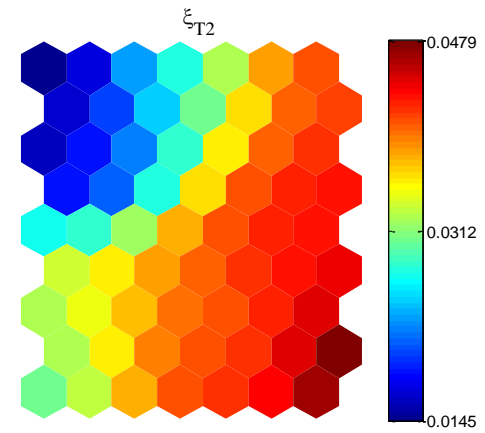

(b)

Fig. 18: Component maps of the optimized damping ratios (a) first TMD tuned to the first mode, (b) second TMD tuned to the second mode 

controlled by the cavity, the obtained result is thus well justified because the best performance of a TMD occur when it acts as a reactive device therefore having small value of the damping ratio.

\subsubsection{Control of the five coupled resonant modes}

In the frequency range of interest, [0-400] $\mathrm{Hz}$, five resonant modes have been observed therefore five TMDs should be used to control the sound field in the cavity. The number of objective functions is five and the total number of parameters, to be optimized, is 25 (five parameters for each of the TMDs); thus, it's clear that the optimization problem, dealing with the control of five modes, is more complicated than the one dealing with the control of two modes.

Preliminary investigations performed during the preparation of the present work showed that carrying out the optimization with the controlled elitist GA, implemented in Matlab, is very computationally expensive and that the convergence is not guaranteed with a reasonable number of generations. Indeed, the results (Pareto front) after more than 2000 generations have not been satisfactory.

To overcome this problem, a hybrid approach [39, 68-70] combining the controlled elitist GA with another local search algorithm can be used. In the work conducted here, the controlled elitist GA has been combined with the goal attainment method [71] implemented in the "fgoalattain" function of Matlab and the obtained results have been satisfactory.

For the evaluations of the five objective functions, the bandwidth parameters are chosen based on the previous conclusions from the single-objective optimizations problems. For the modes controlled by the plate (i.e. $\varpi_{1}=108.59 \mathrm{~Hz}$ and $\varpi_{4}=343.8 \mathrm{~Hz}$ ) the bandwidth is taken $\Delta f=40 \mathrm{~Hz}$. For the other modes controlled by the cavity, the bandwidth is taken $\Delta f=2 \mathrm{~Hz}$ except for the mode corresponding to $\varpi_{3}=313.20 \mathrm{~Hz}$ for which numerical investigations have shown that a bandwidth $\Delta f=10 \mathrm{~Hz}$, instead of $\Delta f=40 \mathrm{~Hz}$, allows obtaining satisfactory results. The initial population size has been set to 800 and the other algorithm's parameters are taken as those set by default in Matlab. After 150 generations, with 387244 function evaluations, the solver has converged to 38 optimal solutions.

Similarly to the previous case with two controlled modes, the obtained solutions and their corresponding optimized objective functions are used in the training of the SOM and then projected onto the twodimensional maps depicted in Fig. 19. The U-matrix in Fig. 19 reveals that the obtained solutions are not evenly distributed and it seems that the Pareto front presents several gaps. Indeed one can observe the presence of two clusters of data (indicated by two red circles in the U-matrix) surrounded by green and red lattices, which means that the Pareto solutions are not evenly distributed.

This result is actually predictable considering the optimization technique that has been used involving hybridization, in which the local search method (i.e. the goal attainment method) doesn't guarantee the diversity of the obtained optimal solutions. 

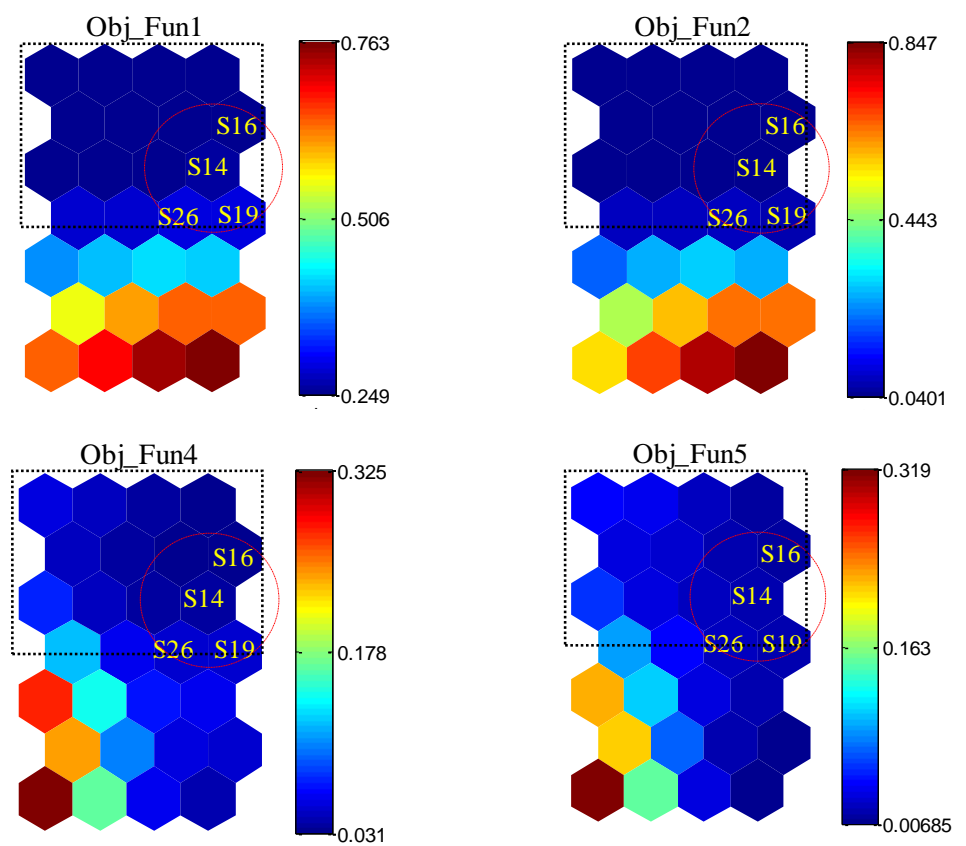

Fig. 19: SOM: the U-matrix and the five objective functions

Fig. 19 also presents the maps of the five objective functions; here too, the conflicting aspect is less highlighted for the same reason related to the diversity of the obtained solutions. Indeed, one can observe a main cluster (indicated by dashed black rectangles in the figure) presenting simultaneously low values of the five objective functions. Consequently, the decision-maker has to choose one solution belonging to this cluster.

Table 9 presents the TMD optimal parameters for the five TMDs and for the four solutions S26, S19, S16 and S14, marked in Fig. 19 by red circles. The results in Table 9 show that for the first TMD (TMD 1), the optimal parameters of the four solutions are very close whereas for the other TMDs, the optimal parameters present relatively large fluctuations, especially in the optimized damping ratios of TMD 2 and TMD 3 which are both dedicated to control two modes that are controlled by the cavity. The same observation can be made for the fourth TMD, dedicated to control the fourth mode corresponding to $\varpi_{4}=344.45 \mathrm{~Hz}$, where it is acting as dissipative device (relatively high value of the damping ratio) for the solution S26, whereas it behaves as a reactive device for the other solutions (very low values of the damping ratios).

The PSD responses of the plate-cavity system equipped by the five TMDs tuned using the obtained optimal solutions S26, S19, S16 and S14 are shown in Fig. 20. The analysis of the obtained PSD responses in Fig. 20 yields that they are roughly the same for the two well separated target frequencies $\varpi_{1}$ and $\varpi_{2}$. This observation doesn't hold for the closely spaced targeted frequencies (i.e. $\varpi_{3}, \varpi_{4}, \varpi_{5}$ ) where significant fluctuations can be observed.

This result can be explained by the fact that for closely spaced targeted frequencies, the coupling effects between the TMDs devices and the vibro-acoustic system is more significant therefore involving important variability of the PSD responses. 


\begin{tabular}{|c|c|c|c|c|c|c|c|c|}
\hline \multirow[b]{2}{*}{ TMDs } & \multirow[b]{2}{*}{$\begin{array}{l}\text { Optimal } \\
\text { solution }\end{array}$} & \multicolumn{5}{|c|}{ TMDs optimal parameters } & \multirow[b]{2}{*}{$\sigma_{p i}^{*}(\mathrm{~Pa})$} & \multirow[b]{2}{*}{$G_{2}(\%)$} \\
\hline & & $\xi_{T}^{*}(\%)$ & $f_{T}^{*}(\mathrm{~Hz})$ & $x_{T M D}^{*}(\mathrm{~m})$ & $y_{T M D}^{*}(\mathrm{~m})$ & $\mu_{T}^{*}(\%)$ & & \\
\hline \multirow{4}{*}{$\begin{array}{c}\text { TMD } 1 \\
\left(\varpi_{1}\right)\end{array}$} & S26 & 8.829 & 98.250 & 0.205 & 0.151 & 1.346 & 0.264 & -67.0 \\
\hline & S19 & 8.360 & 98.217 & 0.203 & 0.147 & 1.363 & 0.268 & -65.5 \\
\hline & S16 & 9.008 & 98.260 & 0.204 & 0.147 & 1.699 & 0.247 & -65.6 \\
\hline & S14 & 8.708 & 98.322 & 0,206 & 0.146 & 1.730 & 0.239 & -63.2 \\
\hline \multirow{4}{*}{$\begin{array}{c}\text { TMD } 2 \\
\left(\varpi_{2}\right)\end{array}$} & S26 & 11.638 & 128.512 & 0.195 & 0,152 & 1.328 & 0.056 & \\
\hline & S19 & 5.320 & 128.580 & 0.195 & 0,151 & 0.918 & 0.061 & \\
\hline & S16 & 4.184 & 128.508 & 0.209 & 0,151 & 0.649 & 0.040 & \\
\hline & S14 & 5.456 & 128.391 & 0.208 & 0,150 & 0.851 & 0.039 & \\
\hline \multirow{4}{*}{$\begin{array}{c}\text { TMD } 3 \\
\left(\varpi_{3}\right)\end{array}$} & S26 & 22.032 & 282.972 & 0.318 & 0.134 & 2.925 & 0.043 & \\
\hline & $\mathrm{S} 19$ & 34.786 & 282.951 & 0.020 & 0.025 & 0.100 & 0.039 & \\
\hline & S16 & 0.026 & 282.947 & 0.270 & 0.160 & 0.502 & 0.026 & \\
\hline & S14 & 0.001 & 282.926 & 0.322 & 0.107 & 1.518 & 0.032 & \\
\hline \multirow{4}{*}{$\begin{array}{c}\text { TMD } 4 \\
\left(\varpi_{4}\right)\end{array}$} & S26 & 2.280 & 311.472 & 0.386 & 0.115 & 0.454 & 0.046 & \\
\hline & S19 & 0.010 & 311.401 & 0.301 & 0.119 & 4.000 & 0.015 & \\
\hline & S16 & 0.010 & 311.418 & 0.310 & 0.118 & 3.790 & 0.027 & \\
\hline & $\mathrm{S} 14$ & 0.010 & 311.337 & 0.300 & 0.123 & 2.595 & 0.030 & \\
\hline \multirow{4}{*}{$\begin{array}{c}\text { TMD } 5 \\
\left(\varpi_{5}\right)\end{array}$} & S26 & 0.559 & 342.640 & 0.057 & 0.090 & 0.877 & 0.004 & \\
\hline & S19 & 2.535 & 342.754 & 0.066 & 0.117 & 0.549 & 0.039 & \\
\hline & $\mathrm{S} 16$ & 2.804 & 342.610 & 0.075 & 0.111 & 0.289 & 0.007 & \\
\hline & S14 & 5.633 & 343.263 & 0.080 & 0.112 & 0.236 & 0.011 & \\
\hline
\end{tabular}

Table 9: Optimal parameters of the five TMDs for different optimal solutions

Looking at the values of the $G_{2}$ index in Table 9, the optimal solution S26 will be, in our opinion, "the best one" for which significant reductions of the PSD response have been recorded for all target frequencies. Indeed, for the first targeted mode, a reduction of $G_{1}=29.12 \mathrm{~dB}$ is reached, whereas it has been $38.95 \mathrm{~dB}, 33.62 \mathrm{~dB}, 26.47 \mathrm{~dB}$ and $36.14 \mathrm{~dB}$, respectively, for the other targeted frequencies as shown in Fig. 20. All these values of the PSD responses attenuation prove the capability of the proposed optimization strategy to handle multimodal control of interior sound under stochastic loading. 


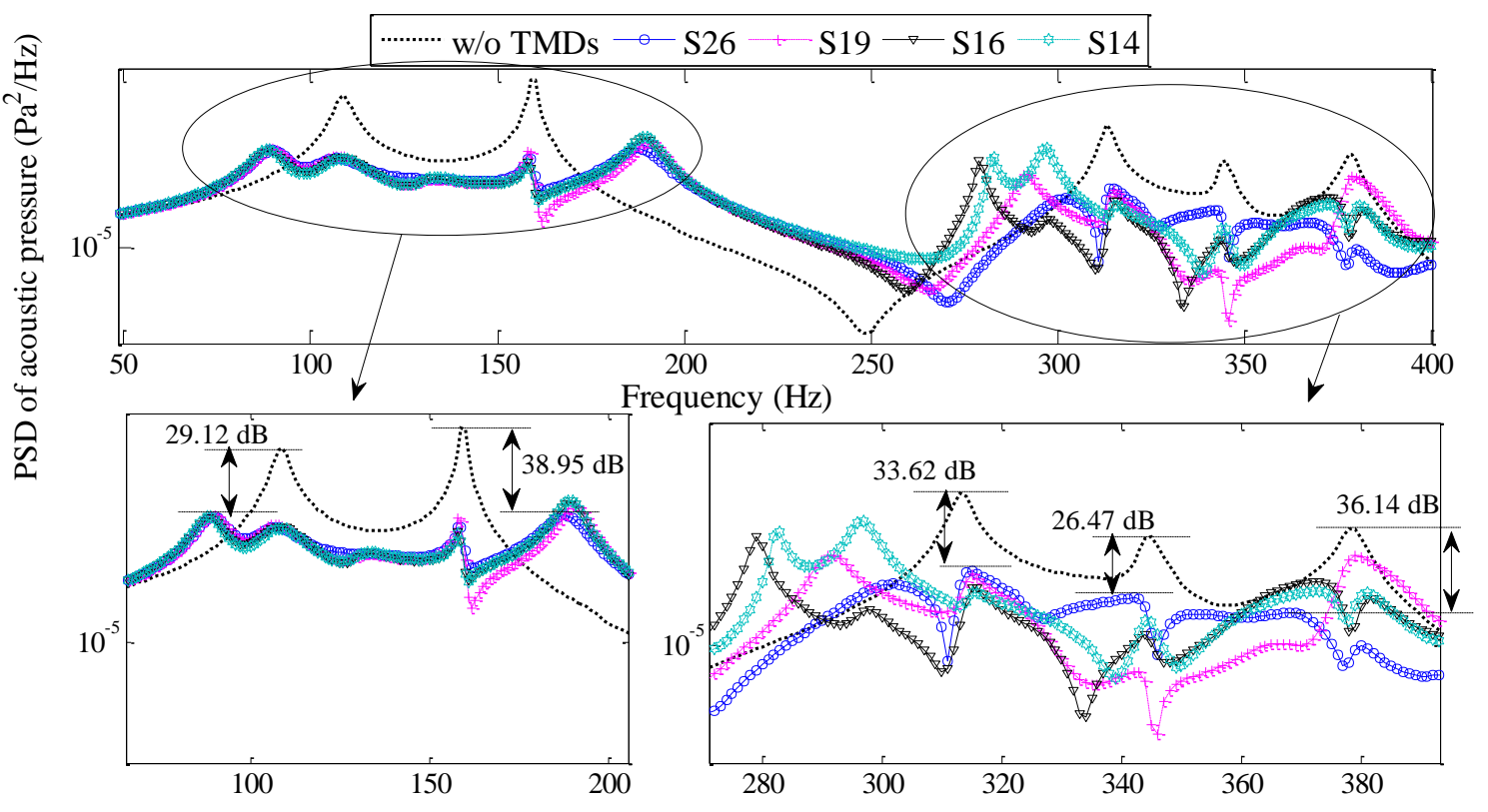

Fig. 20: PSD responses for four optimal solutions (sound control using five TMDs)

\section{Conclusions}

In the work presented, a vibro-acoustic control of random interior sound pressure inside a cavity is proposed. The control of the interior sound pressure has been performed using TMD devices attached to a flexible plate driven by a primary point force with Gaussian white noise characteristics. The plate is attached to a rectangular rigid-walled cavity.

In order to derive the optimal TMD parameters, a strategy based on an acoustic criterion is suggested. The strategy is to reduce the root mean square acoustic pressure in a given location inside the cavity. By making use of a numerical example, the efficiency of the proposed strategy has been investigated and comparisons with other optimization strategies, involving structural and acoustic criteria, have been discussed.

The obtained results show that for the target modes that are dominated by plate's modes, a broadband control can achieve good performance and significant reduction in the PSD responses can be reached when a relatively high mass ratio is used for the TMDs. When a narrowband control is performed, the obtained results show that for well separated target frequencies, undesirable resonant peaks appear and the global performance of the TMD device is limited. On the contrary, when the target frequency is closely spaced to its neighboring resonant frequencies, the appearance of the new resonant peaks is less significant because of the coupling that occurs between the TMD device and the neighboring frequencies. Indeed, for closely spaced natural frequencies, not only the target frequency is affected by the TMD device, but also its neighbors.

For the coupled modes that are controlled by cavity modes, the numerical investigations have shown that a narrowband control can achieve good performance especially for well separated targeted frequencies. For the target frequencies that are closely spaced, a narrowband control can generate undesirable effects on the 
off-target frequencies; a broadband control has significantly improved the global performance of the TMD device.

Except for the coupled mode dominated by a plate mode (with a natural frequency separated from its neighboring), the optimal damping ratios obtained for both modes that are either controlled by the plate or by the cavity, have been relatively small. This result, particularly observed for narrowband control, is in fact coherent with the results obtained in the literature where the TMD device should act as a highly reactive device instead of dissipative device in order to guarantee a maximum sound reduction.

The performance of the proposed strategy is also compared with others, taken from the literature, and the obtained results show its effectiveness and its superiority, for both kinds of coupled modes (i.e. that are dominated by the plate and that are dominated by the cavity). Although the APE strategy shows good performance on the off-target frequencies (for some target frequencies dominated by cavity modes), the obtained results show that it is not suitable concurrently for both kinds of resonant modes, especially in the close vicinity of the target frequencies.

In order to prove the capability of the proposed optimization strategy to deal with multimodal control of interior sound, two TMDs devices have been used to control two resonant coupled modes. The optimization of the TMDs parameters has been performed using a controlled elitist GA and, unlike the single-objective optimization problem where a single optimal solution is obtained, a set of optimal solutions is obtained in a Pareto front. To help the designer in the decision-making, the SOM have been used to visualize the obtained solutions that have been clustered into four clusters allowing easier choice of an acceptable optimal solution. The numerical investigations have shown that an acceptable optimal solution allowed obtaining significant attenuations in PSD responses.

Unlike the case of control of two resonant modes where the controlled elitist GA has been used, the extension of the internal sound control to five resonant modes has required a hybridization method involving the controlled elitist GA along with the goal attainment optimization method. The hybridization has allowed obtaining satisfactory solutions with reasonable computational CPU time.

Similarly to the previous case of control of two modes, the SOM technique has been used and the optimized solutions, obtained for the five targeted modes, have been clustered allowing obtaining acceptable solutions. The numerical investigations have shown that good control attenuations of the interior sound can be achieved for all targeted modes. 


\section{References}

[1] M. Harrison, Vehicle Refinement, Controlling Noise and Vibration in Road Vehicles, 1st ed., Butterworth-Heinemann, Oxford, 2004.

[2] C.Q. Howard, C.H. Hansen, A. Zander, Vibro-acoustic noise control treatments for payload bays of launch vehicles: Discrete to fuzzy solutions, Applied Acoustics, 66 (2005) 1235-1261.

[3] C.Q. Howard, C.H. Hansen, Investigation of Passive Control Devices for Potential Application to a Launch Vehicle Structure to Reduce the Interior Noise Levels During Launch. Final Report for Stage 4,Task 4.5, The University of Adelaide, Adelaide, SA 5000, Australia, 2006, pp. 87.

[4] S.J. Estève, M.E. Johnson, Reduction of sound transmission into a circular cylindrical shell using distributed vibration absorbers and Helmholtz resonators, The Journal of the Acoustical Society of America, 112 (2002) 2840-2848.

[5] C.R. Fuller, J.P. Maillard, M. Mercadal, A.H. von Flotow, Control of aircraft interior noise using globally detuned vibration absorbers, Journal of Sound and Vibration, 203 (1997) 745-761.

[6] J. Marty, F. Chris, M. Pierre, O. Haisam, Optimization of distributed vibration absorbers for sound transmission into a composite cylinder, 7th AIAA/CEAS Aeroacoustics Conference and Exhibit, American Institute of Aeronautics and Astronautics, 2001.

[7] P. Gardonio, Review of Active Techniques for Aerospace Vibro-Acoustic Control, Journal of Aircraft, 39 (2002) 206-214.

[8] R.I. Wright, M.R.F. Kidner, Vibration Absorbers: A Review of Applications in Interior Noise Control of Propeller Aircraft, Journal of Vibration and Control, 10 (2004) 1221-1237.

[9] S.K. Lau, S.K. Tang, Sound fields in a slightly damped rectangular enclosure under active control, Journal of Sound and Vibration, 238 (2000) 637-660.

[10] Q. Lin, Z. Liu, Q. Wang, Active control of structural acoustic pressure in a rectangular cavity using piezoelectric actuators, European Journal of Mechanics - A/Solids, 20 (2001) 573-583.

[11] G. Rosi, J. Pouget, F. dell'Isola, Control of sound radiation and transmission by a piezoelectric plate with an optimized resistive electrode, European Journal of Mechanics - A/Solids, 29 (2010) 859-870.

[12] Z.S. Liu, H.P. Lee, C. Lu, Passive and active interior noise control of box structures using the structural intensity method, Applied Acoustics, 67 (2006) 112-134.

[13] B. Philip, J.B. Michael, J.E. Stephen, Vibration control using an adaptive tuned vibration absorber with a variable curvature stiffness element, Smart Materials and Structures, 14 (2005) 1055.

[14] P. Gardonio, M. Zilletti, Integrated tuned vibration absorbers: A theoretical study, The Journal of the Acoustical Society of America, 134 (2013) 3631-3644.

[15] P. Gardonio, S. Miani, F. Blanchini, D. Casagrande, S.J. Elliott, Plate with decentralised velocity feedback loops: Power absorption and kinetic energy considerations, Journal of Sound and Vibration, 331 (2012) 1722-1741.

[16] P. Bonello, Adaptive Tuned Vibration Absorbers: Design Principles, Concepts and Physical Implementation, in: F. Beltran-Carbajal (Ed.) Vibration Analysis and Control - New Trends and Developments, InTech, Croatia, 2011, pp. 1-26.

[17] M. Zilletti, S.J. Elliott, E. Rustighi, Optimisation of dynamic vibration absorbers to minimise kinetic energy and maximise internal power dissipation, Journal of Sound and Vibration, 331 (2012) 4093-4100. [18] J.P.D. Hartog, Mechanical Vibrations, McGraw-Hill, New York, 1985.

[19] S.S. Kuik, C.Q. Howard, C.H. Hansen, A.C. Zander, Tuned vibration absorbers for control of noise radiated by a panel, ACOUSTICS Adelaide, Australia, 2009.

[20] C.Q. Howard, C.H. Hansen, A.C. Zander, Optimisation of Design and Location of Acoustic and Vibration Absorbers Using a Distributed Computing Network, Proceeding of Acoustics, Busselton, Western Australia, 2005. 
[21] W. Ho, B. Wong, D. England, Tuned Mass Damper for Rail Noise Control, in: T. Maeda, P.-E. Gautier, C.E. Hanson, B. Hemsworth, J.T. Nelson, B. Schulte-Werning, D. Thompson, P. de Vos (Eds.) Noise and Vibration Mitigation for Rail Transportation Systems: Proceedings of the 10th International Workshop on RailwayNoise, Nagahama, Japan, 18-22 October 2010, Springer Japan, Tokyo, 2012, pp. 89-96.

[22] B. Tiseo, A. Concilio, S. Ameduri, A. Gianvito, A shape memory alloys based tuneable dynamic vibration absorber for vibration tonal control, Journal of Theoretical and applied mechanics, 48 (2010) 135-153.

[23] E. Turco, P. Gardonio, On the use of tuneable mass dampers for broadband noise control in a cylindrical enclosure, The 22nd International Congress on Sound and Vibration, Florence, Italy, 2015.

[24] S.J. Estève, M.E. Johnson, Adaptive Helmholtz resonators and passive vibration absorbers for cylinder interior noise control, Journal of Sound and Vibration, 288 (2005) 1105-1130.

[25] S.V. Gorin, M.V. Kuklin, Size and acoustic optimization of Helmholtz resonators, Russian Engineering Research, 32 (2012) 115-117.

[26] F.J. Fahy, C. Schofield, A note on the interaction between a Helmholtz resonator and an acoustic mode of an enclosure, Journal of Sound and Vibration, 72 (1980) 365-378.

[27] F. Fahy, P. Gardonio, 8 - Introduction to Numerically Based Analyses of Fluid-Structure Interaction, Sound and Structural Vibration (Second Edition), Academic Press, Oxford, 2007, pp. 449-519.

[28] B. Ben Smida, R. Majed, N. Bouhaddi, M. Ouisse, Investigations for a model reduction technique of fluid-structure coupled systems, Proceedings of the Institution of Mechanical Engineers, Part C: Journal of Mechanical Engineering Science, (2011).

[29] G.B. Warburton, Optimum absorber parameters for various combinations of response and excitation parameters, Earthquake Engineering \& Structural Dynamics, 10 (1982) 381-401.

[30] G.B. Warburton, E.O. Ayorinde, Optimum absorber parameters for simple systems, Earthquake Engineering \& Structural Dynamics, 8 (1980) 197-217.

[31] R. D'Amico, K. Koo, C.C. Claeys, B. Pluymers, W. Desmet, Optimal dynamic vibration absorber design for minimizing the band-averaged input power using the residue theorem, Journal of Sound and Vibration, 338 (2015) 60-75.

[32] R. Masopust, Dynamic absorbers of vibrations. theory and technical applications by B. G. Korenev, L. M. Reznikov, Nauka, Moscow Moscow, 1988. No. of pages: 303. (in Russia), Earthquake Engineering \& Structural Dynamics, 16 (1988) 1233-1233.

[33] F. Fahy, P. Gardonio, 7 - Acoustic Coupling between Structures and Enclosed Volumes of Fluid, Sound and Structural Vibration (Second Edition), Academic Press, Oxford, 2007, pp. 403-448.

[34] S. Obayashi, D. Sasaki, Multi-objective optimization for aerodynamic designs by using ARMOGAs, Proceedings. Seventh International Conference on High Performance Computing and Grid in Asia Pacific Region, 2004., 2004, pp. 396-403.

[35] I. Giagkiozis, P.J. Fleming, Methods for multi-objective optimization: An analysis, Information Sciences, 293 (2015) 338-350.

[36] M.R. Farmani, J. Roshanian, M. Babaie, P.M. Zadeh, Multi-objective collaborative multidisciplinary design optimization using particle swarm techniques and fuzzy decision making, Proceedings of the Institution of Mechanical Engineers, Part C: Journal of Mechanical Engineering Science, 226 (2012) 22812295.

[37] G. Droandi, G. Gibertini, Aerodynamic blade design with multi-objective optimization for a tiltrotor aircraft, Aircraft Engineering and Aerospace Technology, 87 (2015) 19-29.

[38] G. Chiandussi, M. Codegone, S. Ferrero, F.E. Varesio, Comparison of multi-objective optimization methodologies for engineering applications, Computers \& Mathematics with Applications, 63 (2012) 912-942.

[39] K. Deb, D. Kalyanmoy, Multi-Objective Optimization Using Evolutionary Algorithms, John Wiley I\&amp; Sons, Inc., 2001. 
[40] G.C. Marano, R. Greco, S. Sgobba, A comparison between different robust optimum design approaches: Application to tuned mass dampers, Probabilistic Engineering Mechanics, 25 (2010) 108118.

[41] C.Q. Howard, B.S. Cazzolato, Fluid-Structure Interaction, Acoustic Analyses Using Matlab and Ansys, CRC Press, 2014, pp. 533-600.

[42] A.W. Leissa, Vibration of Plates, NASA, Washington, DC, United States, 1969, pp. 362.

[43] S.A. Hambric, J.B. Fahnline, Sound-Structure Interaction Fundamentals, Engineering Vibroacoustic Analysis, John Wiley \& Sons, Ltd, 2016, pp. 88-113.

[44] S.H. Sung, D.J. Nefske, Structural-Acoustic Finite-Element Analysis for Interior Acoustics, Engineering Vibroacoustic Analysis, John Wiley \& Sons, Ltd, 2016, pp. 144-178.

[45] C. Yang, D. Li, L. Cheng, Dynamic vibration absorbers for vibration control within a frequency band, Journal of Sound and Vibration, 330 (2011) 1582-1598.

[46] S.H. Sung, D.J. Nefske, Interior and Exterior Sound, Engineering Vibroacoustic Analysis, John Wiley \& Sons, Ltd, 2016, pp. 52-87.

[47] P. Gardonio, Boundary Layer Noise - Part 2: Interior Noise Radiation and Control, in: R. Camussi (Ed.) Noise Sources in Turbulent Shear Flows: Fundamentals and Applications, Springer Vienna, Vienna, 2013, pp. 379-448.

[48] J. Li, J. Chen, Stochastic Processes and Random Fields, Stochastic Dynamics of Structures, John Wiley \& Sons, Ltd, 2010, pp. 7-41.

[49] E. VanMarcke, Spectral Parameters, Level Crossings, and Extremes, Random Fields, WORLD SCIENTIFIC, 2010, pp. 145-188.

[50] J. Sólnes, Stochastic Processes and Random Vibrations: Theory and Practice, by John Wiley \& Sons Ltd, , Baffins Lane, Chichester, 1997.

[51] S. Marburg, M. Shepherd, S.A. Hambric, Structural-Acoustic Optimization, Engineering Vibroacoustic Analysis, John Wiley \& Sons, Ltd, 2016, pp. 268-304.

[52] S. Marburg, Developments in structural-acoustic optimization for passive noise control, Archives of Computational Methods in Engineering, 9 (2002) 291-370.

[53] C. Pal, I. Hagiwara, Dynamic analysis of a coupled structural-acoustic problem: Simultaneous multimodal reduction of vehicle interior noise level by combined optimization, Finite Elements in Analysis and Design, 14 (1993) 225-234.

[54] E. Yuksel, G. Kamci, I. Basdogan, Vibro-Acoustic Design Optimization Study to Improve the Sound Pressure Level Inside the Passenger Cabin, Journal of Vibration and Acoustics, 134 (2012) 061017061017-061019.

[55] S. Marburg, H.-J. Hardtke, Efficient optimization of a noise transfer function by modification of a shell structure geometry - Part II: Application to a vehicle dashboard, Struct Multidisc Optim, 24 (2002) 60-71.

[56] K. Deb, T. Goel, Controlled Elitist Non-dominated Sorting Genetic Algorithms for Better Convergence, Proceedings of the First International Conference on Evolutionary Multi-Criterion Optimization, Springer-Verlag, 2001, pp. 67-81.

[57] M.R. Jolly, J.Q. Sun, Passive tuned vibration absorbers for sound radiation reduction from vibrating panels, Journal of Sound and Vibration, 191 (1996) 577-583.

[58] X. Blasco, J.M. Herrero, J. Sanchis, M. Martínez, A new graphical visualization of n-dimensional Pareto front for decision-making in multiobjective optimization, Information Sciences, 178 (2008) 39083924.

[59] S. Parashar, V. Pediroda, C. Poloni, Self Organizing Maps (SOM) for Design Selection in Robust MultiObjective Design of Aerofoil, 46th AIAA Aerospace Sciences Meeting and Exhibit, American Institute of Aeronautics and Astronautics, 2008. 
[60] S. Parashar, N. Fateh, V. Pediroda, C. Poloni, Self Organizing Maps (SOM) for Design Selection in Multi-Objective Optimization using modeFRONTIER, SAE International, 2008.

[61] T. Kohonen, The Basic SOM, Self-Organizing Maps, Springer Berlin Heidelberg, Berlin, Heidelberg, 2001, pp. 105-176.

[62] T. Kohonen, The self-organizing map, Neurocomputing, 21 (1998) 1-6.

[63] J.H. Esa Alhoniemi, Juha Parhankangas and Juha Vesanto, SOM Toolbox, http://www.cis.hut.fi/projects/somtoolbox/download/ (accessed 02.01.2017), 2005.

[64] N.K. Jain, V.R. Sonti, Structural Acoustics of a Rectangular Panel Backed by a Cavity: An Analytical Matrix Approach, Journal of Vibration and Acoustics, 139 (2017) 031004-031004-031008.

[65] S.K. Lau, S.K. Tang, Sound fields in a rectangular enclosure under active sound transmission control, The Journal of the Acoustical Society of America, 110 (2001) 925-938.

[66] D.A. Rade, V. Steffen, Optimization of dynamic vibration absorbers over a frequency band, Mechanical Systems and Signal Processing, 14 (2000) 679-690.

[67] M. Abé, T. Igusa, Tuned mass dampers for structures with closely spaced natural frequencies, Earthquake Engineering \& Structural Dynamics, 24 (1995) 247-261.

[68] C.A.C. Coello, G.B. Lamont, D.A.V. Veldhuizen, Evolutionary Algorithms for Solving Multi-Objective Problems (Genetic and Evolutionary Computation), Springer-Verlag New York, Inc., 2006.

[69] K. Amouzgar, Multi-Objective Optimization using Genetic Algorithms, School of Engineering in Jönköping, University of Jönköping, Sweden, , 2012, pp. 79.

[70] D.E. Goldberg, Genetic Algorithms in Search, Optimization and Machine Learning, Addison-Wesley Longman Publishing Co., Inc., 1989.

[71] F. Gembicki, Y. Haimes, Approach to performance and sensitivity multiobjective optimization: The goal attainment method, IEEE Transactions on Automatic Control, 20 (1975) 769-771. 


\begin{tabular}{ccc}
\hline Parameter & & Value \\
\cline { 1 - 1 }$l_{x}(\mathrm{~m})$ & & $0.5 \mathrm{~m}$ \\
$l_{y}(\mathrm{~m})$ & & $0.3 \mathrm{~m}$ \\
$l_{z}(\mathrm{~m})$ & & $1.1 \mathrm{~m}$ \\
$h(\mathrm{~m})$ & & $0.003 \mathrm{~m}$ \\
$E(\mathrm{~Pa})$ & & $70 \times 10^{9} \mathrm{~Pa}$ \\
$\rho_{s}$ (unit) & & $2700 \mathrm{~kg} \cdot \mathrm{m}^{-3}$ \\
$v$ & & 0.3 \\
$\rho_{0}$ (unit) & & $1.21 \mathrm{~kg} \cdot \mathrm{m}^{-3}$ \\
$c_{0}$ (unit) & & $344 \mathrm{~m} \cdot \mathrm{s}^{-1}$ \\
\hline
\end{tabular}

Table 1: Numerical values of the parameters of the vibro-acoustic system 


\begin{tabular}{|c|c|c|c|c|c|c|}
\hline \multicolumn{2}{|c|}{ In-vacuo plate } & \multicolumn{2}{|c|}{ Rigid-walled cavity } & \multicolumn{3}{|c|}{ Coupled plate-cavity } \\
\hline Modes & $\begin{array}{l}\text { Frequencies } \\
(\mathrm{Hz})\end{array}$ & Modes & $\begin{array}{c}\text { Frequencies } \\
(\mathrm{Hz})\end{array}$ & $\begin{array}{c}\text { Frequencies } \\
(\mathrm{Hz})\end{array}$ & $\begin{array}{c}\text { Mode } \\
\text { controlled by }\end{array}$ & Modes involved \\
\hline \multirow{3}{*}{$(1,1)$} & \multirow{3}{*}{110.43} & $(0,0,0)$ & 0 & 0 & & \\
\hline & & & & 108,59 & plate & $(1,1)-(0,0,0)$ \\
\hline & & $(0,0,1)$ & 156.36 & 159,52 & cavity & $(1,1)-(0,0,1)$ \\
\hline \multirow[t]{2}{*}{$(2,1)$} & \multirow[t]{2}{*}{198.12} & & & 196,52 & plate & $(2,1)-(1,0,0)$ \\
\hline & & $(0,0,2)$ & 312.72 & 313,20 & cavity & $(3,1)-(0,0,2)$ \\
\hline \multirow[t]{2}{*}{$(3,1)$} & \multirow[t]{2}{*}{344.27} & & & 343,80 & plate & $(3,1)-(0,0,2)$ \\
\hline & & $(1,0,0)$ & 344.00 & 344,45 & cavity & $(2,1)-(1,0,0)$ \\
\hline \multirow[t]{2}{*}{$(1,2)$} & \multirow[t]{2}{*}{354.01} & & & 352,35 & plate & $(1,2)-(0,1,0)$ \\
\hline & & $(1,0,1)$ & 377.86 & 378,61 & cavity & $(2,1)-(1,0,1)$ \\
\hline
\end{tabular}

Table 2: Natural modes and frequencies of the uncoupled and coupled plate-cavity system 


\begin{tabular}{|c|c|c|c|c|c|c|c|}
\hline \multirow[b]{2}{*}{$\mu(\%)$} & \multirow[b]{2}{*}{$\Delta f(\mathrm{~Hz})$} & \multicolumn{4}{|c|}{ TMD optimal parameters } & \multirow{2}{*}{$\sigma_{p}^{*}(\mathrm{~Pa})$} & \multirow{2}{*}{$G_{2}(\%)$} \\
\hline & & $\xi_{T}^{*}(\%)$ & $f_{T}^{*}(\mathrm{~Hz})$ & $x_{c}^{*}(\mathrm{~m})$ & $y_{c}^{*}(\mathrm{~m})$ & & \\
\hline \multirow{7}{*}{2} & 2 & 0.01 & 110.897 & 0.240 & 0.150 & 0.0027 & 15.8 \\
\hline & 10 & 0.03 & 111.106 & 0.244 & 0.150 & 0.0320 & 15.6 \\
\hline & 20 & 1.16 & 110.694 & 0.244 & 0.150 & 0.1231 & 4.0 \\
\hline & 40 & 13.45 & 110.919 & 0.253 & 0.151 & 0.3284 & -19.2 \\
\hline & Korenev & 12.97 & 110.425 & $* * *$ & $* * *$ & $* * *$ & -18.6 \\
\hline & Den Hartog & 16.66 & 102.248 & $* * *$ & $* * *$ & $* * *$ & -21.5 \\
\hline & APE & 0.96 & 108.529 & 0.232 & 0.108 & $* * *$ & 4.4 \\
\hline \multirow{7}{*}{3} & 2 & 0.01 & 111.739 & 0.235 & 0.149 & 0.0018 & 21.6 \\
\hline & 10 & 0.01 & 112.238 & 0.241 & 0.149 & 0.0209 & 23.9 \\
\hline & 20 & 0.37 & 112.107 & 0.241 & 0.150 & 0.0715 & 31.3 \\
\hline & 40 & 14.96 & 110.612 & 0.252 & 0.151 & 0.2729 & -22.8 \\
\hline & Korenev & 15.25 & 110.425 & $* * *$ & $* * *$ & $* * *$ & -23.0 \\
\hline & Den Hartog & 20.04 & 98.595 & $* * *$ & $* * *$ & $* * *$ & -25.1 \\
\hline & APE & 0.09 & 114.294 & 0.234 & 0.110 & $* * *$ & -0.9 \\
\hline \multirow{7}{*}{4} & 2 & 0.01 & 112.405 & 0.229 & 0.149 & 0.0014 & 26.7 \\
\hline & 10 & 0.01 & 113.273 & 0.238 & 0.149 & 0.0155 & 16.8 \\
\hline & 20 & 0.17 & 113.304 & 0.238 & 0.149 & 0.0505 & 26.3 \\
\hline & 40 & 15.06 & 110.168 & 0.248 & 0.150 & 0.2301 & -25.5 \\
\hline & Korenev & 16.94 & 110.425 & $* * *$ & $* * *$ & $* * *$ & -27.8 \\
\hline & Den Hartog & 22.74 & 95.197 & $* * *$ & $* * *$ & $* * *$ & -28.3 \\
\hline & APE & 0.45 & 108.554 & 0.146 & 0.160 & $* * *$ & -15.8 \\
\hline
\end{tabular}

Table 3: Optimal TMD parameters for different mass ratio and with different bandwidth control, $\varpi_{1}=108.59 \mathrm{~Hz}$ 


\begin{tabular}{|c|c|c|c|c|c|c|c|}
\hline \multirow[b]{2}{*}{$\mu(\%)$} & \multirow[b]{2}{*}{$\Delta f(\mathrm{~Hz})$} & \multicolumn{4}{|c|}{ TMD optimal parameters } & \multirow{2}{*}{$\sigma_{p}^{*}(\mathrm{~Pa})$} & \multirow[b]{2}{*}{$G_{2}(\%)$} \\
\hline & & $\xi_{T}^{*}(\%)$ & $f_{T}^{*}(\mathrm{~Hz})$ & $x_{T M D}^{*}(\mathrm{~m})$ & $y_{T M D}^{*}(\mathrm{~m})$ & & \\
\hline \multirow{7}{*}{2} & 2 & 2.234 & 337.493 & 0.031 & 0.097 & $4.66 \times 10^{-05}$ & 4.1 \\
\hline & 10 & 2.053 & 338.687 & 0.047 & 0.067 & $1.04 \times 10^{-03}$ & 9.9 \\
\hline & 20 & 1.984 & 340.100 & 0.051 & 0.061 & $4.13 \times 10^{-03}$ & 10.9 \\
\hline & 40 & 3.588 & 334.259 & 0.055 & 0.064 & $2.29 \times 10^{-02}$ & 5.2 \\
\hline & Korenev & 7.646 & 344.266 & $* * *$ & $* * *$ & $* * *$ & 6.7 \\
\hline & Den Hartog & 9.509 & 335.966 & $* * *$ & $* * *$ & $* * *$ & 4.7 \\
\hline & APE & 3.9861 & 331.598 & 0.049 & 0.079 & $* * *$ & 47.4 \\
\hline \multirow{7}{*}{3} & 2 & 2.930 & 333.262 & 0.035 & 0.088 & $4.46 \times 10^{-05}$ & 4.7 \\
\hline & 10 & 3.617 & 331.804 & 0.041 & 0.079 & $1.12 \times 10^{-03}$ & 6.2 \\
\hline & 20 & 1.923 & 340.319 & 0.051 & 0.057 & $2.94 \times 10^{-03}$ & 6.7 \\
\hline & 40 & 3.316 & 335.858 & 0.053 & 0.061 & $1.71 \times 10^{-02}$ & 5.8 \\
\hline & Korenev & 8.202 & 344.266 & $* * *$ & $* * *$ & $* * *$ & 6.8 \\
\hline & Den Hartog & 10.224 & 334.669 & $* * *$ & $* * *$ & $* * *$ & 5.8 \\
\hline & APE & 4.5208 & 329.270 & 0.048 & 0.075 & $* * *$ & 17.7 \\
\hline \multirow{7}{*}{4} & 2 & 2,878 & 337.802 & 0.023 & 0.107 & $5.42 \times 10^{-05}$ & 6.3 \\
\hline & 10 & 2,113 & 337.686 & 0.048 & 0.060 & $5.97 \times 10^{-04}$ & 6.5 \\
\hline & 20 & 1,829 & 340.713 & 0.051 & 0.055 & $2.31 \times 10^{-03}$ & 6.6 \\
\hline & 40 & 3,017 & 337.149 & 0.053 & 0.058 & $1.38 \times 10^{-02}$ & 7.0 \\
\hline & Korenev & 9,199 & 344.266 & $* * *$ & $* * *$ & $* * *$ & 7.6 \\
\hline & Den Hartog & 11,523 & 332.078 & $* * *$ & $* * *$ & $* * *$ & 7.2 \\
\hline & APE & 0.120 & 342.984 & 0.049 & 0.053 & $* * *$ & 8.6 \\
\hline
\end{tabular}

Table 4: Optimal TMD parameters for different mass ratio and with different bandwidth control, $\varpi_{4}=344.45 \mathrm{~Hz}$ 


\begin{tabular}{|c|c|c|c|c|c|c|c|}
\hline \multirow[b]{2}{*}{$\mu(\%)$} & \multirow[b]{2}{*}{$\Delta f(\mathrm{~Hz})$} & \multicolumn{4}{|c|}{ TMD optimal parameters } & \multirow{2}{*}{$\sigma_{p}^{*} \times 10^{-4}(\mathrm{~Pa})$} & \multirow{2}{*}{$G_{2}(\%)$} \\
\hline & & $\xi_{T}^{*}(\%)$ & $f_{T}^{*}(\mathrm{~Hz})$ & $x_{T M D}^{*}(\mathrm{~m})$ & $y_{T M D}^{*}(\mathrm{~m})$ & & \\
\hline \multirow{5}{*}{2} & 2 & 0.693 & 155.554 & 0.192 & 0.147 & 77.094 & -33.8 \\
\hline & 10 & 0.676 & 153.279 & 0.141 & 0.132 & 725.867 & -21.5 \\
\hline & 20 & 0.010 & 175.472 & 0.261 & 0.156 & 1249.856 & -8.3 \\
\hline & 40 & 0.010 & 184.877 & 0.275 & 0.156 & 2103.574 & -19.7 \\
\hline & APE & 0.6708 & 155.794 & 0.176 & 0.172 & $* * *$ & -8.6 \\
\hline \multirow{5}{*}{3} & 2 & 1.043 & 152.277 & 0.184 & 0.146 & 38.186 & -38.0 \\
\hline & 10 & 1.026 & 150.374 & 0.151 & 0.136 & 365.339 & -30.1 \\
\hline & 20 & 0.010 & 175.424 & 0.247 & 0.157 & 883.536 & -26.4 \\
\hline & 40 & 0.010 & 183.097 & 0.256 & 0.157 & 1408.895 & -19.7 \\
\hline & APE & 0.519 & 163.519 & 0.222 & 0.157 & $* * *$ & -21.9 \\
\hline \multirow{5}{*}{4} & 2 & 1.341 & 149.323 & 0.180 & 0.145 & 19.371 & -41.1 \\
\hline & 10 & 1.355 & 147.578 & 0.158 & 0.139 & 189.293 & -36.5 \\
\hline & 20 & 1.185 & 146.967 & 0.146 & 0.132 & 503.278 & -32.0 \\
\hline & 40 & 0.010 & 178.974 & 0.243 & 0.158 & 1028.770 & -39.9 \\
\hline & APE & 3.324 & 166.133 & 0.217 & 0.182 & $* * *$ & -25.4 \\
\hline
\end{tabular}

Table 5: Optimal TMD parameters for different mass ratio and with different bandwidth control, $\varpi_{2}=159.52 \mathrm{~Hz}$ 


\begin{tabular}{|c|c|c|c|c|c|c|c|}
\hline \multirow[b]{2}{*}{$\mu(\%)$} & \multirow[b]{2}{*}{$\Delta f(\mathrm{~Hz})$} & \multicolumn{4}{|c|}{ TMD optimal parameters } & \multirow{2}{*}{$\sigma_{p}^{*} \times 10^{-4}(\mathrm{~Pa})$} & \multirow[b]{2}{*}{$G_{2}(\%)$} \\
\hline & & $\xi_{T}^{*}(\%)$ & $f_{T}^{*}(\mathrm{~Hz})$ & $x_{T M D}^{*}(\mathrm{~m})$ & $y_{T M D}^{*}(\mathrm{~m})$ & & \\
\hline \multirow{5}{*}{2} & 2 & 0.022 & 321.830 & 0.417 & 0.173 & 0.789 & 28.3 \\
\hline & 10 & 0.090 & 320.331 & 0.426 & 0.168 & 21.580 & 8.1 \\
\hline & 20 & 0.186 & 318.665 & 0.431 & 0.162 & 74.52473 & 8.2 \\
\hline & 40 & 0.366 & 307.183 & 0.066 & 0.093 & 254.940 & 8.9 \\
\hline & APE & 0.159 & 309.059 & 0.046 & 0.081 & $* * *$ & 0.3 \\
\hline \multirow{5}{*}{3} & 2 & 0.010 & 325.745 & 0.446 & 0.163 & 1.215 & 9.6 \\
\hline & 10 & 0.010 & 325.621 & 0.446 & 0.164 & 19.427 & 8.8 \\
\hline & 20 & 0.010 & 325.750 & 0.445 & 0.166 & 65.375 & 7.9 \\
\hline & 40 & 0.464 & 305.144 & 0.062 & 0.093 & 165.545 & 14.1 \\
\hline & APE & 0.194 & 307.598 & 0.043 & 0.082 & $* * *$ & 5.6 \\
\hline \multirow{5}{*}{4} & 2 & 0.010 & 328.523 & 0.456 & 0.158 & 1.382 & 9.5 \\
\hline & 10 & 0.010 & 328.386 & 0.456 & 0.159 & 19.187 & 8.6 \\
\hline & 20 & 0.010 & 328.903 & 0.454 & 0.163 & 63.263 & 7.7 \\
\hline & 40 & 0.551 & 302.808 & 0.059 & 0.092 & 125.255 & 19.2 \\
\hline & APE & 0.065 & 313.775 & 0.060 & 0.051 & $* * *$ & 5.0 \\
\hline
\end{tabular}

Table 6: Optimal TMD parameters for different mass ratio and with different bandwidth control, $\varpi_{3}=313.20 \mathrm{~Hz}$ 


\begin{tabular}{|c|c|c|c|c|c|c|c|}
\hline \multirow[b]{2}{*}{$\mu(\%)$} & \multirow[b]{2}{*}{$\Delta f(\mathrm{~Hz})$} & \multicolumn{4}{|c|}{ TMD optimal parameters } & \multirow{2}{*}{$\sigma_{p}^{*} \times 10^{-4}(\mathrm{~Pa})$} & \multirow[b]{2}{*}{$G_{2}(\%)$} \\
\hline & & $\xi_{T}^{*}(\%)$ & $f_{T}^{*}(\mathrm{~Hz})$ & $x_{T M D}^{*}(\mathrm{~m})$ & $y_{T M D}^{*}(\mathrm{~m})$ & & \\
\hline \multirow{5}{*}{2} & 2 & 2.163 & 365.795 & 0.064 & 0.071 & 0.242 & 2.8 \\
\hline & 10 & 2.173 & 366.019 & 0.067 & 0.067 & 7,077 & 14.6 \\
\hline & 20 & 2.122 & 378.299 & 0.048 & 0.104 & 27.392 & 25.1 \\
\hline & 40 & 1.915 & 366.677 & 0.062 & 0.070 & 63.377 & 27.5 \\
\hline & APE & 0.854 & 371.300 & 0.080 & 0.046 & $* * *$ & 2.7 \\
\hline \multirow{5}{*}{3} & 2 & 2.143 & 363.097 & 0.057 & 0.071 & 0.187 & 20.7 \\
\hline & 10 & 2.310 & 374.864 & 0.092 & 0.035 & 12.680 & 13.9 \\
\hline & 20 & 2.128 & 377.796 & 0.041 & 0.104 & 24.377 & 17.5 \\
\hline & 40 & 1.744 & 416.471 & 0.040 & 0.134 & 63.690 & 15.3 \\
\hline & APE & 2.624 & 360.625 & 0.060 & 0.072 & $* * *$ & 13.9 \\
\hline \multirow{5}{*}{4} & 2 & 2.216 & 366.139 & 0.070 & 0.049 & 1.122 & 22.2 \\
\hline & 10 & 2.177 & 363.564 & 0.064 & 0.056 & 5.292 & 13.9 \\
\hline & 20 & 2.134 & 362.738 & 0.061 & 0.060 & 14.528 & 19.8 \\
\hline & 40 & 1.923 & 363.275 & 0.057 & 0.064 & 42.348 & 12.1 \\
\hline & APE & 0.990 & 381.270 & 0.028 & 0.111 & $* * *$ & 15.1 \\
\hline
\end{tabular}

Table 7: Optimal TMD parameters for different mass ratio and with different bandwidth control, $\varpi_{5}=378.61 \mathrm{~Hz}$ 


\begin{tabular}{|c|c|c|c|c|c|c|c|c|}
\hline \multirow[b]{2}{*}{ TMDs } & \multirow[b]{2}{*}{$\begin{array}{l}\text { Optimal } \\
\text { solution }\end{array}$} & \multicolumn{5}{|c|}{ TMDs optimal parameters } & \multirow[b]{2}{*}{$\sigma_{p i}^{*}(\mathrm{~Pa})$} & \multirow[b]{2}{*}{$G_{2}(\%)$} \\
\hline & & $\xi_{T}^{*}(\%)$ & $f_{T}^{*}(\mathrm{~Hz})$ & $x_{T M D}^{*}(\mathrm{~m})$ & $y_{T M D}^{*}(\mathrm{~m})$ & $\mu_{T}^{*}(\%)$ & & \\
\hline \multirow{4}{*}{ TMD 1} & S8 & 8.451 & 99.279 & 0.185 & 0.140 & 3.038 & 0.201 & -62.7 \\
\hline & S60 & 6.505 & 99.483 & 0.209 & 0.147 & 3.184 & 0.100 & 0.9 \\
\hline & S79 & 7.257 & 99.417 & 0.202 & 0.146 & 3.092 & 0.112 & -26.4 \\
\hline & S99 & 8.333 & 99.370 & 0.193 & 0.142 & 3.061 & 0.152 & -50.2 \\
\hline \multirow{4}{*}{ TMD 2} & S8 & 2.065 & 142.276 & 0.175 & 0.138 & 3.991 & 0.067 & \\
\hline & S60 & 3.785 & 130.691 & 0.257 & 0.152 & 3.834 & 0.413 & \\
\hline & S79 & 4.024 & 130.778 & 0.229 & 0.151 & 3.937 & 0.302 & \\
\hline & S99 & 4.221 & 138.563 & 0.208 & 0.146 & 3.963 & 0.169 & \\
\hline
\end{tabular}

Table 8: Optimal parameters of the two TMDs for different optimal solutions 


\begin{tabular}{|c|c|c|c|c|c|c|c|c|}
\hline \multirow[b]{2}{*}{ TMDs } & \multirow[b]{2}{*}{$\begin{array}{l}\text { Optimal } \\
\text { solution }\end{array}$} & \multicolumn{5}{|c|}{ TMDs optimal parameters } & \multirow[b]{2}{*}{$\sigma_{p i}^{*}(\mathrm{~Pa})$} & \multirow[b]{2}{*}{$G_{2}(\%)$} \\
\hline & & $\xi_{T}^{*}(\%)$ & $f_{T}^{*}(\mathrm{~Hz})$ & $x_{T M D}^{*}(\mathrm{~m})$ & $y_{T M D}^{*}(\mathrm{~m})$ & $\mu_{T}^{*}(\%)$ & & \\
\hline \multirow{4}{*}{$\begin{array}{c}\text { TMD } 1 \\
\left(\varpi_{1}\right)\end{array}$} & S26 & 8.829 & 98.250 & 0.205 & 0.151 & 1.346 & 0.264 & -67.0 \\
\hline & $\mathrm{S} 19$ & 8.360 & 98.217 & 0.203 & 0.147 & 1.363 & 0.268 & -65.5 \\
\hline & $\mathrm{S} 16$ & 9.008 & 98.260 & 0.204 & 0.147 & 1.699 & 0.247 & -65.6 \\
\hline & $\mathrm{S} 14$ & 8.708 & 98.322 & 0,206 & 0.146 & 1.730 & 0.239 & -63.2 \\
\hline \multirow{4}{*}{$\begin{array}{c}\text { TMD } 2 \\
\left(\varpi_{2}\right)\end{array}$} & S26 & 11.638 & 128.512 & 0.195 & 0,152 & 1.328 & 0.056 & \\
\hline & S19 & 5.320 & 128.580 & 0.195 & 0,151 & 0.918 & 0.061 & \\
\hline & S16 & 4.184 & 128.508 & 0.209 & 0,151 & 0.649 & 0.040 & \\
\hline & $\mathrm{S} 14$ & 5.456 & 128.391 & 0.208 & 0,150 & 0.851 & 0.039 & \\
\hline \multirow{4}{*}{$\begin{array}{c}\text { TMD } 3 \\
\left(\varpi_{3}\right)\end{array}$} & S26 & 22.032 & 282.972 & 0.318 & 0.134 & 2.925 & 0.043 & \\
\hline & $\mathrm{S} 19$ & 34.786 & 282.951 & 0.020 & 0.025 & 0.100 & 0.039 & \\
\hline & S16 & 0.026 & 282.947 & 0.270 & 0.160 & 0.502 & 0.026 & \\
\hline & $\mathrm{S} 14$ & 0.001 & 282.926 & 0.322 & 0.107 & 1.518 & 0.032 & \\
\hline \multirow{4}{*}{$\begin{array}{c}\text { TMD } 4 \\
\left(\varpi_{4}\right)\end{array}$} & S26 & 2.280 & 311.472 & 0.386 & 0.115 & 0.454 & 0.046 & \\
\hline & S19 & 0.010 & 311.401 & 0.301 & 0.119 & 4.000 & 0.015 & \\
\hline & S16 & 0.010 & 311.418 & 0.310 & 0.118 & 3.790 & 0.027 & \\
\hline & S14 & 0.010 & 311.337 & 0.300 & 0.123 & 2.595 & 0.030 & \\
\hline \multirow{4}{*}{$\begin{array}{c}\text { TMD } 5 \\
\left(\varpi_{5}\right)\end{array}$} & S26 & 0.559 & 342.640 & 0.057 & 0.090 & 0.877 & 0.004 & \\
\hline & S19 & 2.535 & 342.754 & 0.066 & 0.117 & 0.549 & 0.039 & \\
\hline & S16 & 2.804 & 342.610 & 0.075 & 0.111 & 0.289 & 0.007 & \\
\hline & $\mathrm{S} 14$ & 5.633 & 343.263 & 0.080 & 0.112 & 0.236 & 0.011 & \\
\hline
\end{tabular}

Table 9: Optimal parameters of the five TMDs for different optimal solutions 


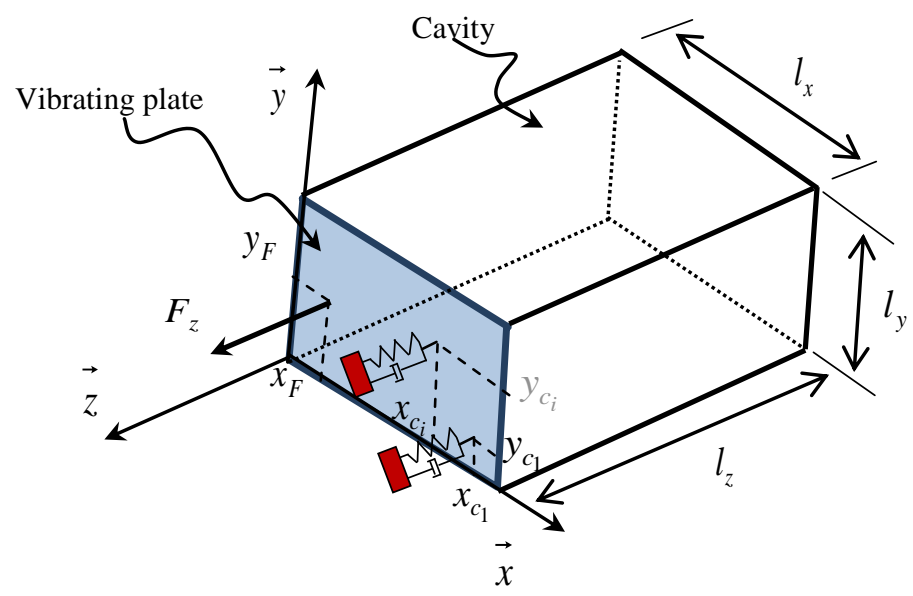

Fig. 1: Plate-cavity system with the attached multi-TMDs devices 


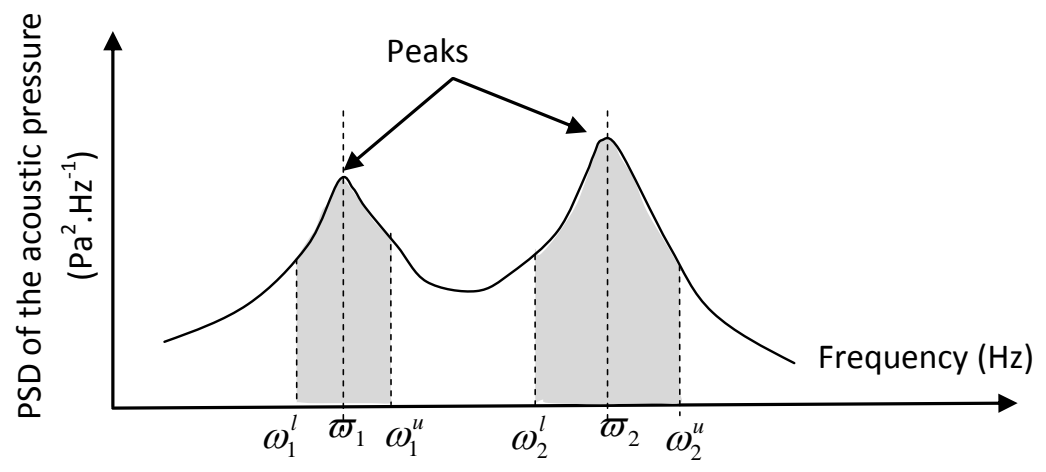

Fig. 2 : schematic representation of two objective functions (i.e. the square roots of the shaded surfaces) 

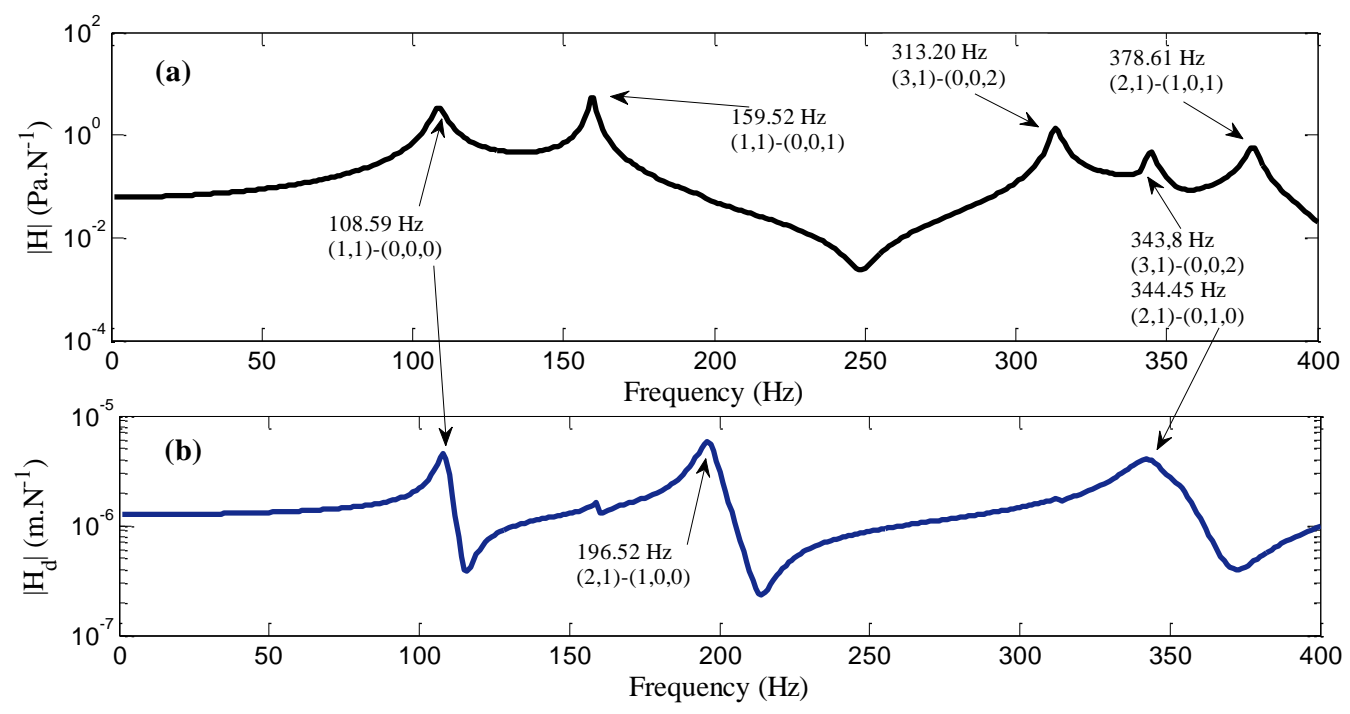

Fig. 3: FRF for the force location $(0.05 \mathrm{~m}, 0.05 \mathrm{~m})$; (a) Pressure response, microphone location $(0.35 \mathrm{~m}, 0.10 \mathrm{~m}$, $0.875 \mathrm{~m})$; (b) displacement response at force location 


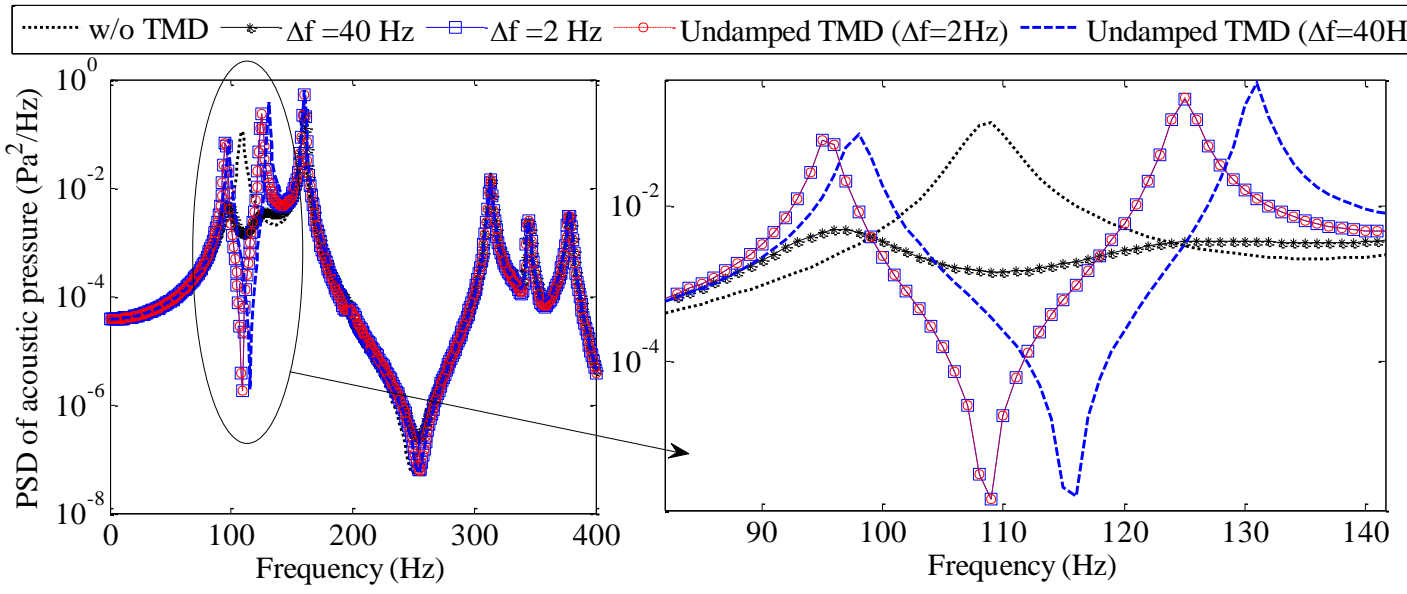

Fig. 4 PSD responses of acoustic pressure for the damped and undamped TMD, $\mu=2 \%, \varpi_{1}=108.59 \mathrm{~Hz}$ 


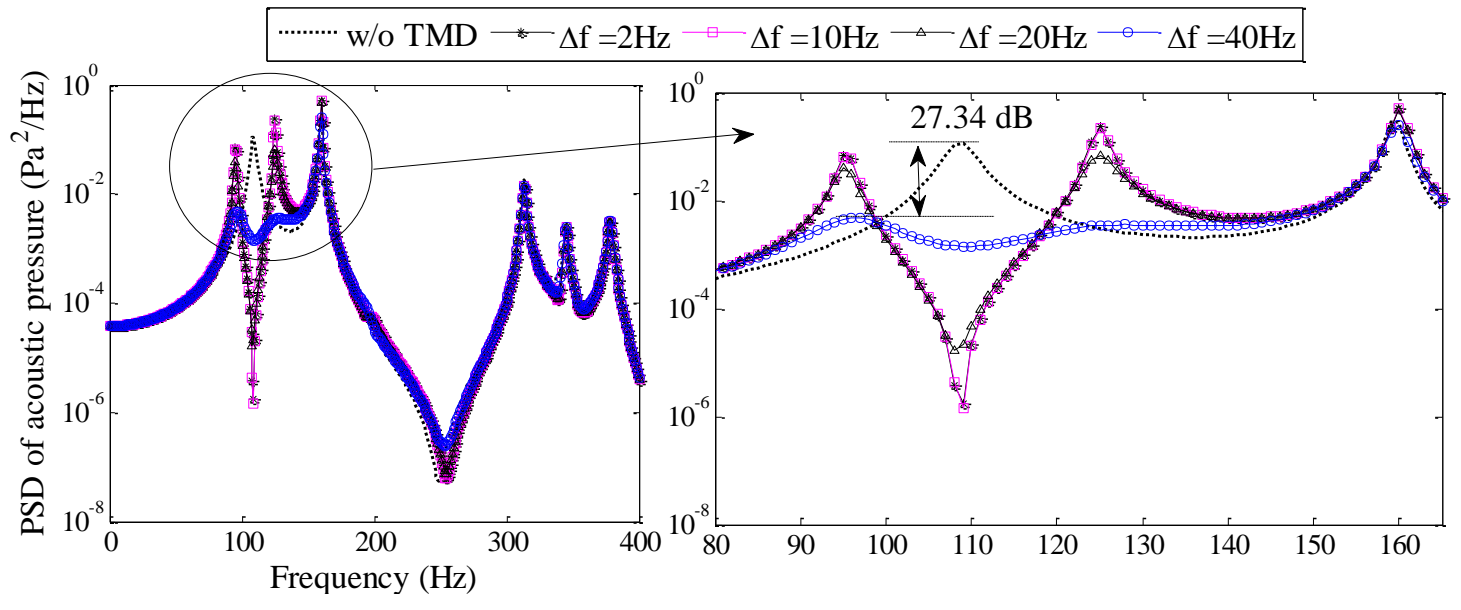

Fig. 5: PSD responses of acoustic pressure for narrowband and broadband control, $\boldsymbol{\mu}=\mathbf{2 \%}, \varpi_{1}=108.59 \mathrm{~Hz}$ 


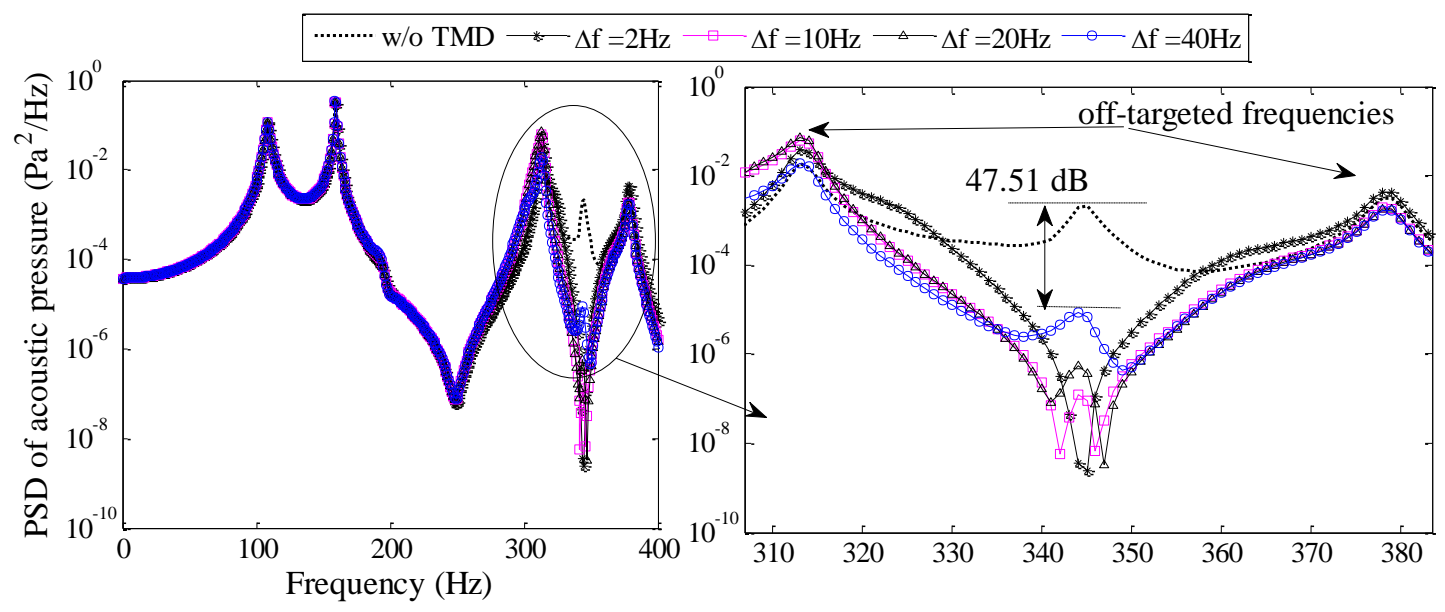

Fig. 6: PSD responses of acoustic pressure for narrowband and broadband control, $\mu=2 \%, \varpi_{4}=343.8 \mathrm{~Hz}$ 


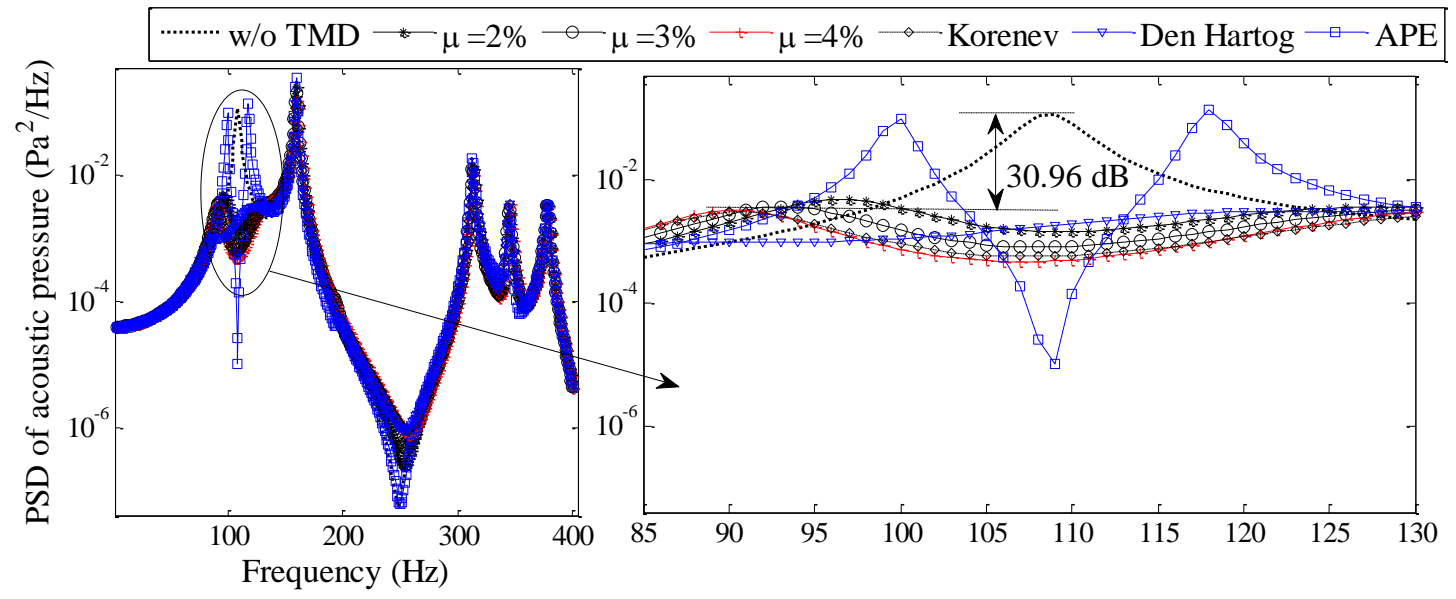

Fig. 7: PSD responses of acoustic pressure for a broadband control $(\Delta \mathbf{f}=\mathbf{4 0 H z})$ and different mass ratios, $\varpi_{1}=108.59 \mathrm{~Hz}$ 


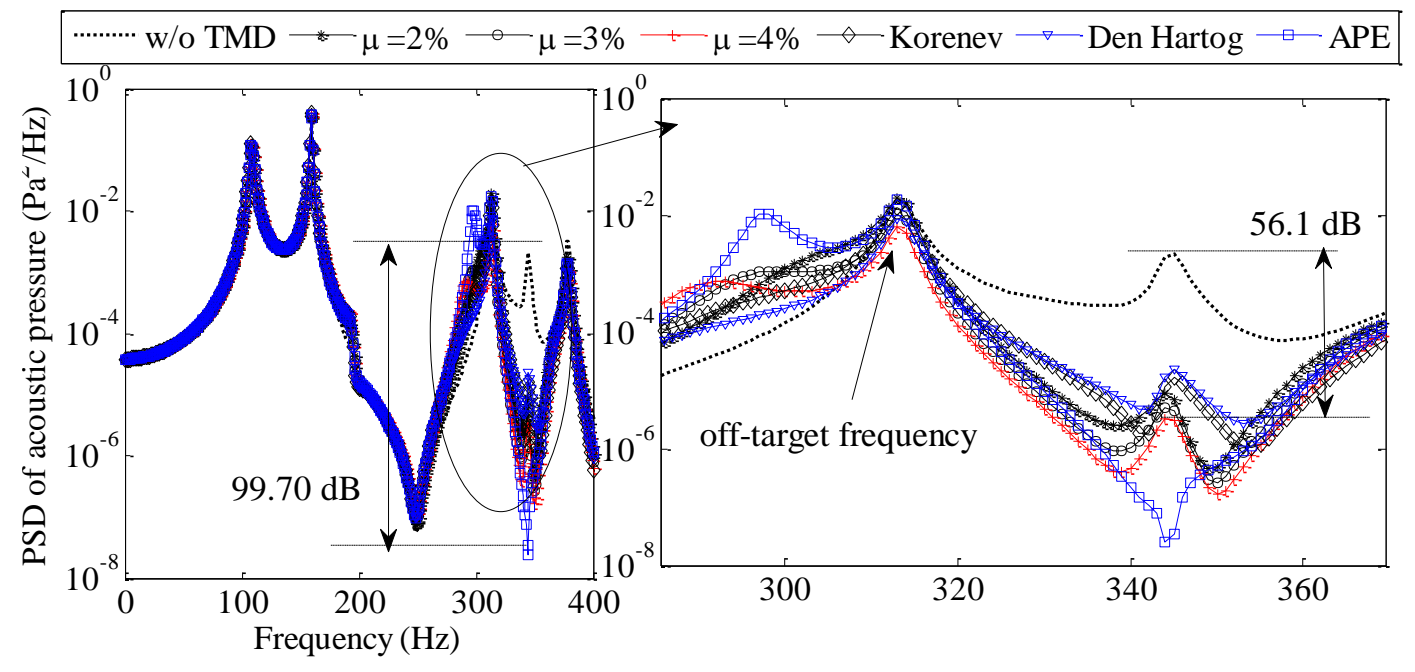

Fig. 8: PSD responses for a broadband control $(\Delta \mathbf{f}=\mathbf{4 0 H z})$ and different mass ratios;, $\varpi_{4}=343.8 \mathrm{~Hz}$ 


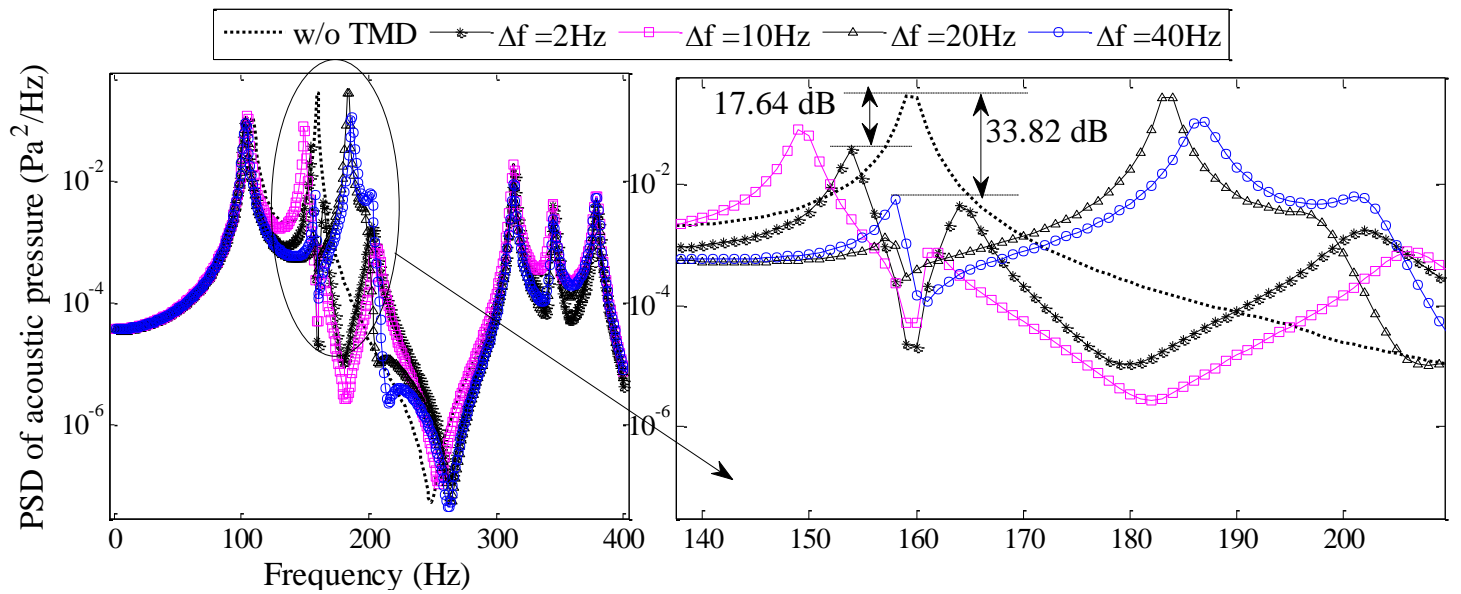

Fig. 9: PSD of acoustic pressure for narrowband and broadband control, $\mu=2 \%, \varpi_{2}=159.52 \mathrm{~Hz}$ 


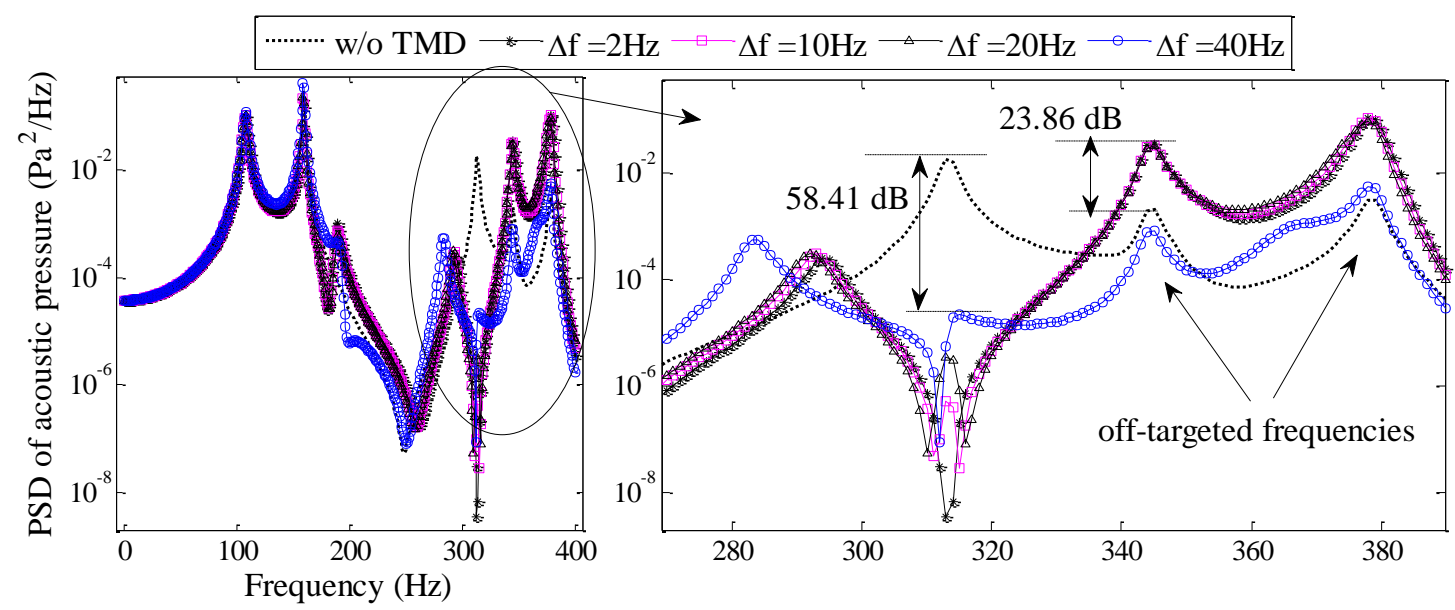

Fig. 10: PSD responses of acoustic pressure for different bandwidth parameter $\Delta \mathbf{f}, \mu=2 \%, \varpi_{3}=313.20 \mathrm{~Hz}$ 


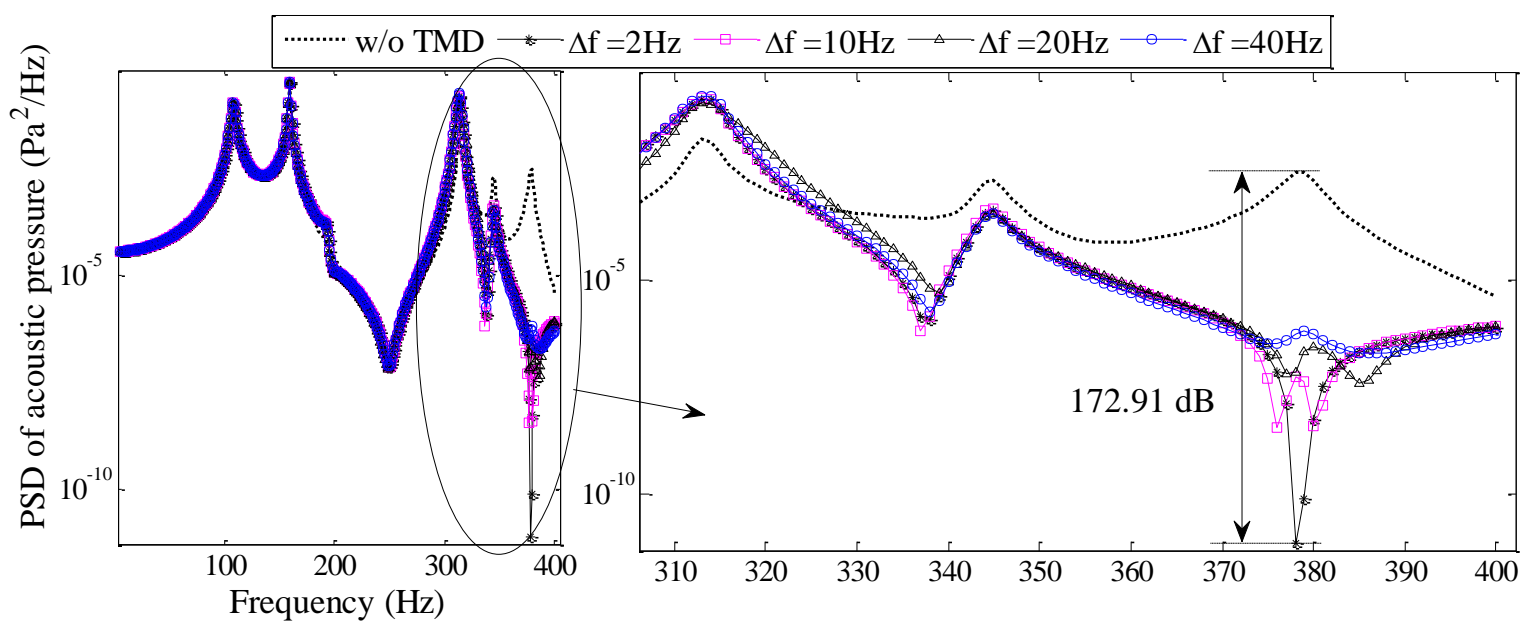

Fig. 11: PSD of acoustic responses for different values $\Delta \mathbf{f}, \mu=2 \%, \varpi_{5}=378.61 \mathrm{~Hz}$ 


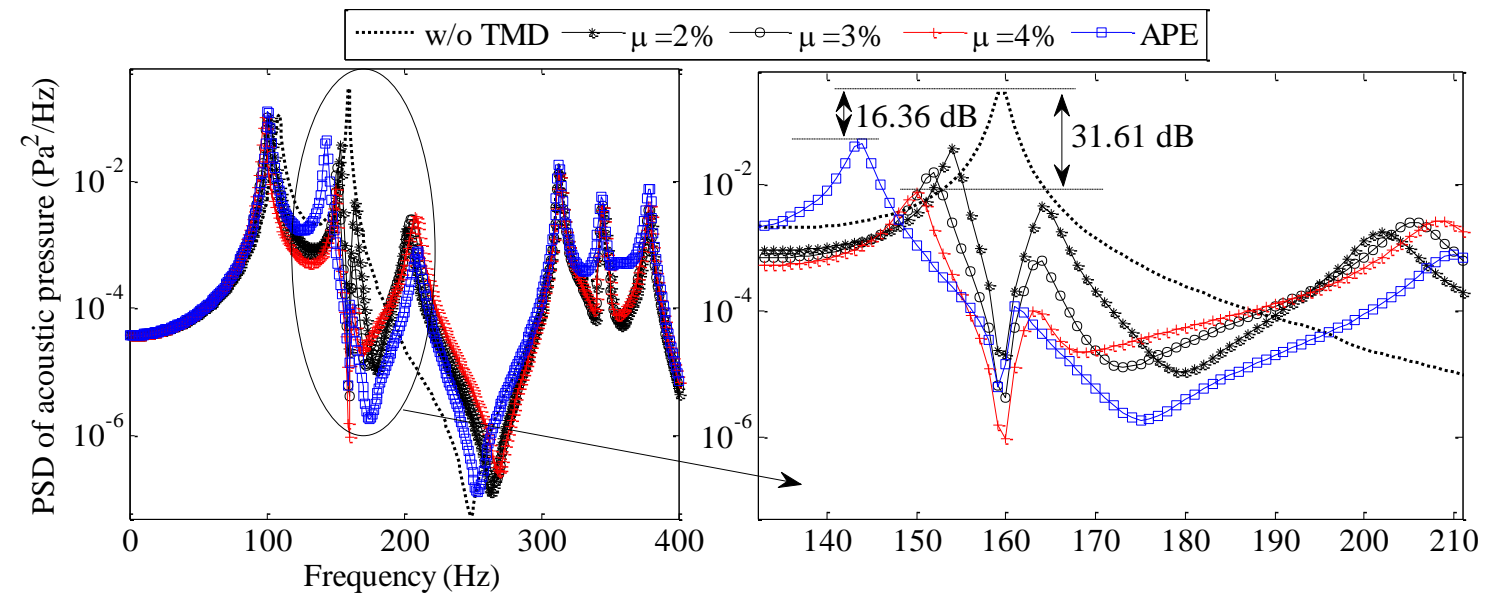

Fig. 12: PSD responses of the acoustic pressure for different mass ratio; $\Delta \mathbf{f}=\mathbf{2 H z} ; \varpi_{2}=159.52 \mathrm{~Hz}$ 


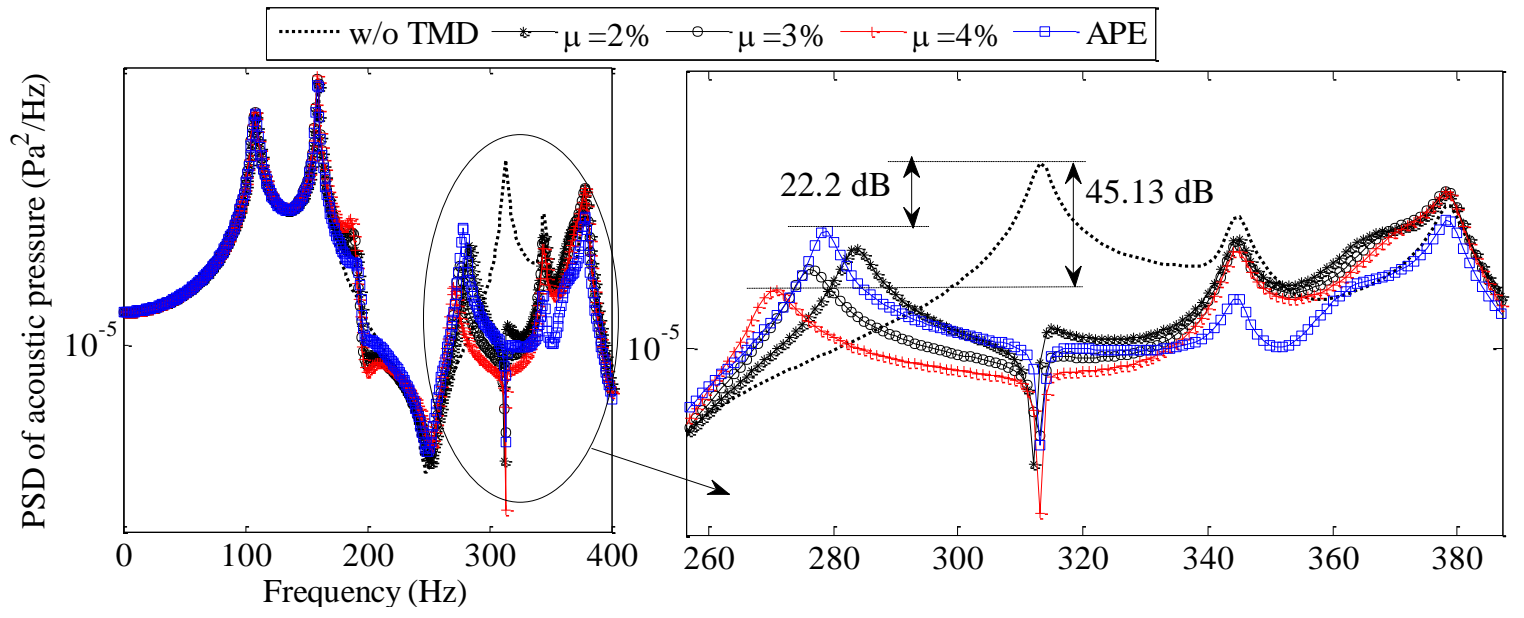

Fig. 13: PSD of acoustic responses for different mass ratio $\mu, \Delta \mathbf{f}=\mathbf{4 0 H z} ; \varpi_{3}=313.20 \mathrm{~Hz}$ 


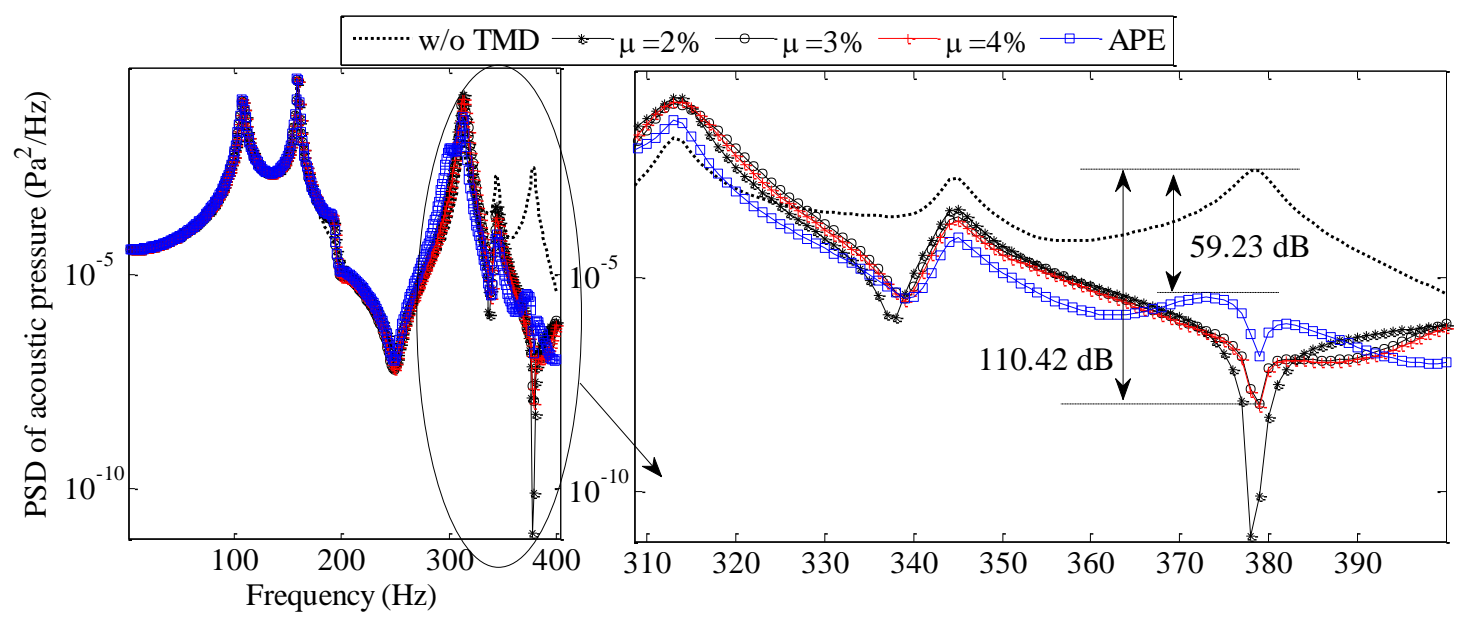

Fig. 14: PSD responses of acoustic pressure for narrowband control $(\Delta \mathrm{f}=\mathbf{2 H z})$ for different values of the mass ratio; $\varpi_{5}=378.61 \mathrm{~Hz}$ 


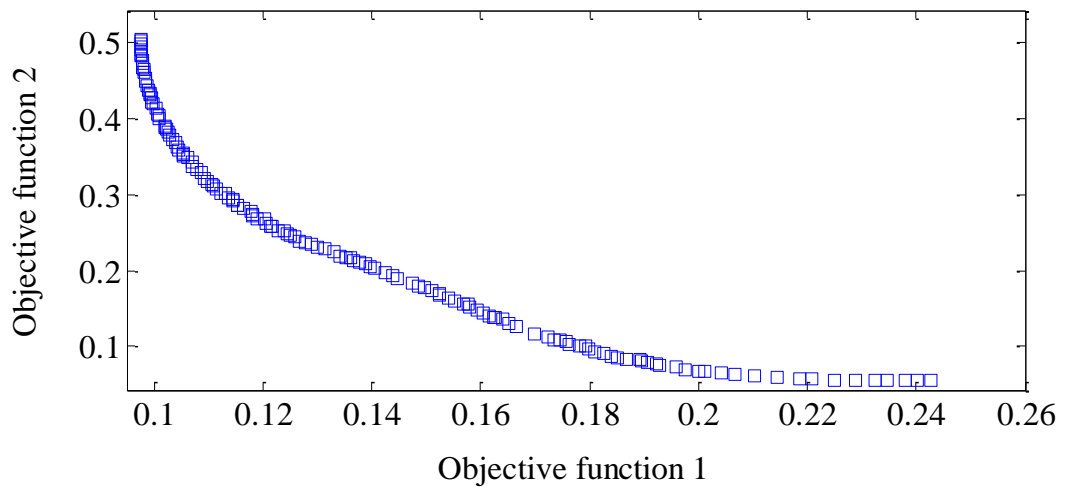

Fig. 15: Pareto front (two objective functions) 


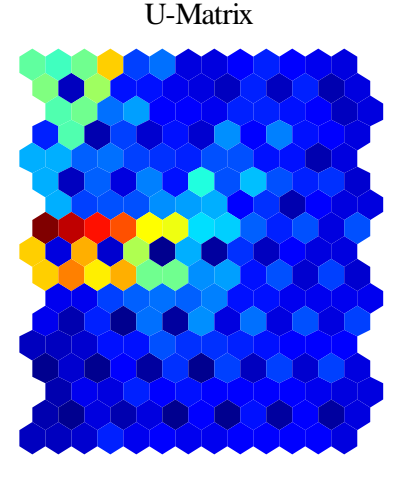

(a)
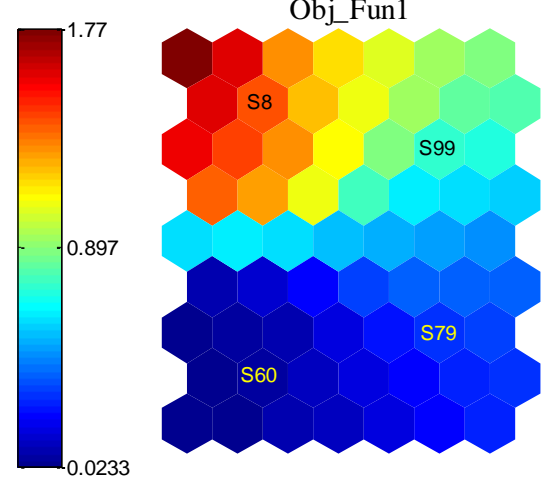

(b)
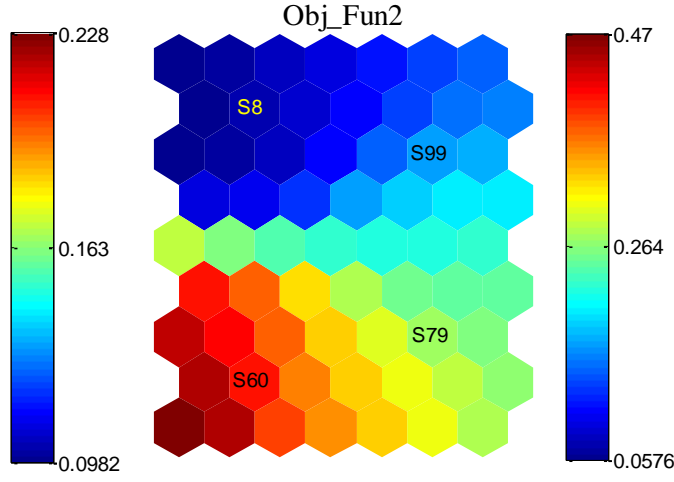

(c)

Fig. 16: SOM; (a) U-matrix map, (b) Objective function one component map; (c) Objective function two component map 


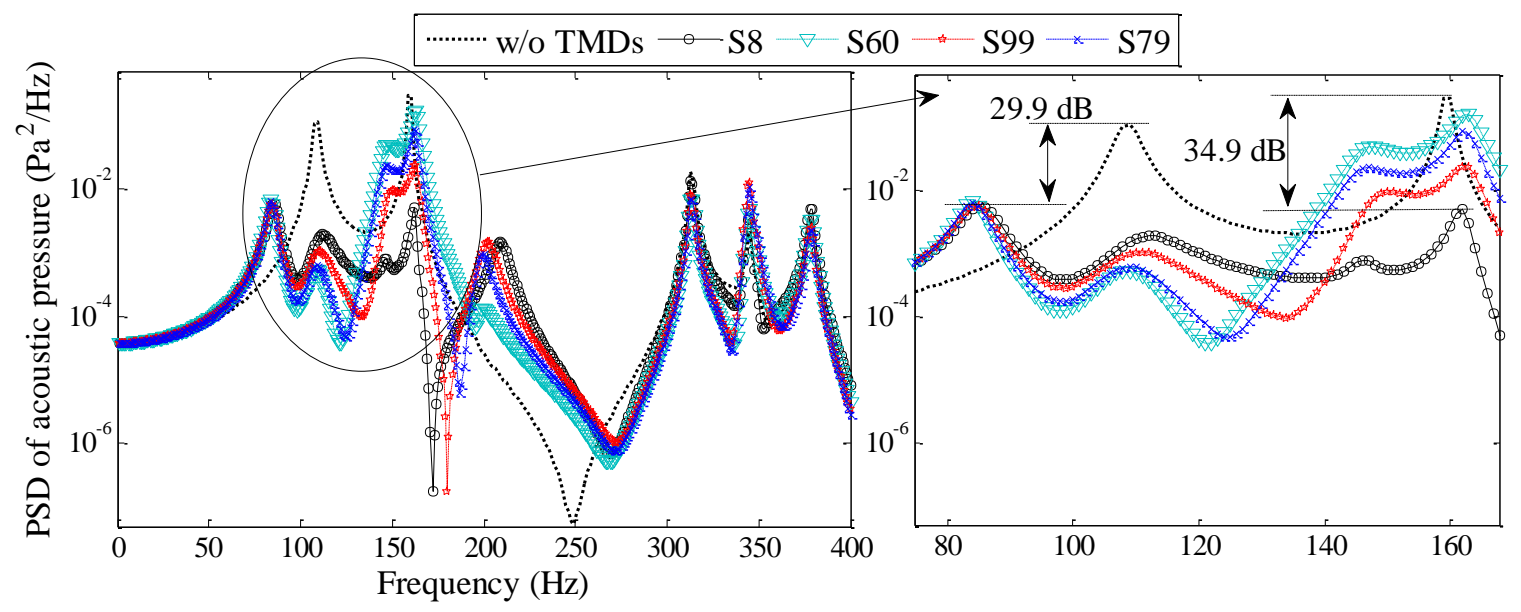

Fig. 17: PSD responses of the four selected optimal solutions (sound control using two TMDs) 


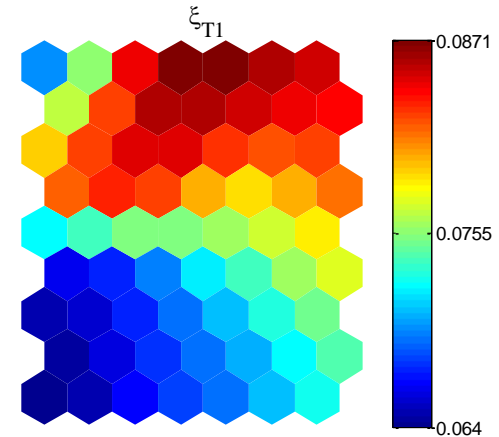

(a)

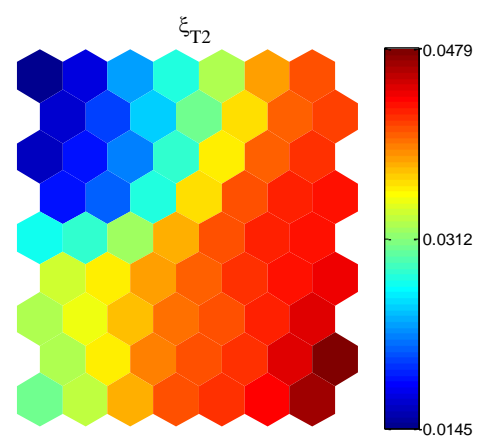

(b)

Fig. 18: Component maps of the optimized damping ratios (a) first TMD tuned to the first mode, (b) second TMD tuned to the second mode 

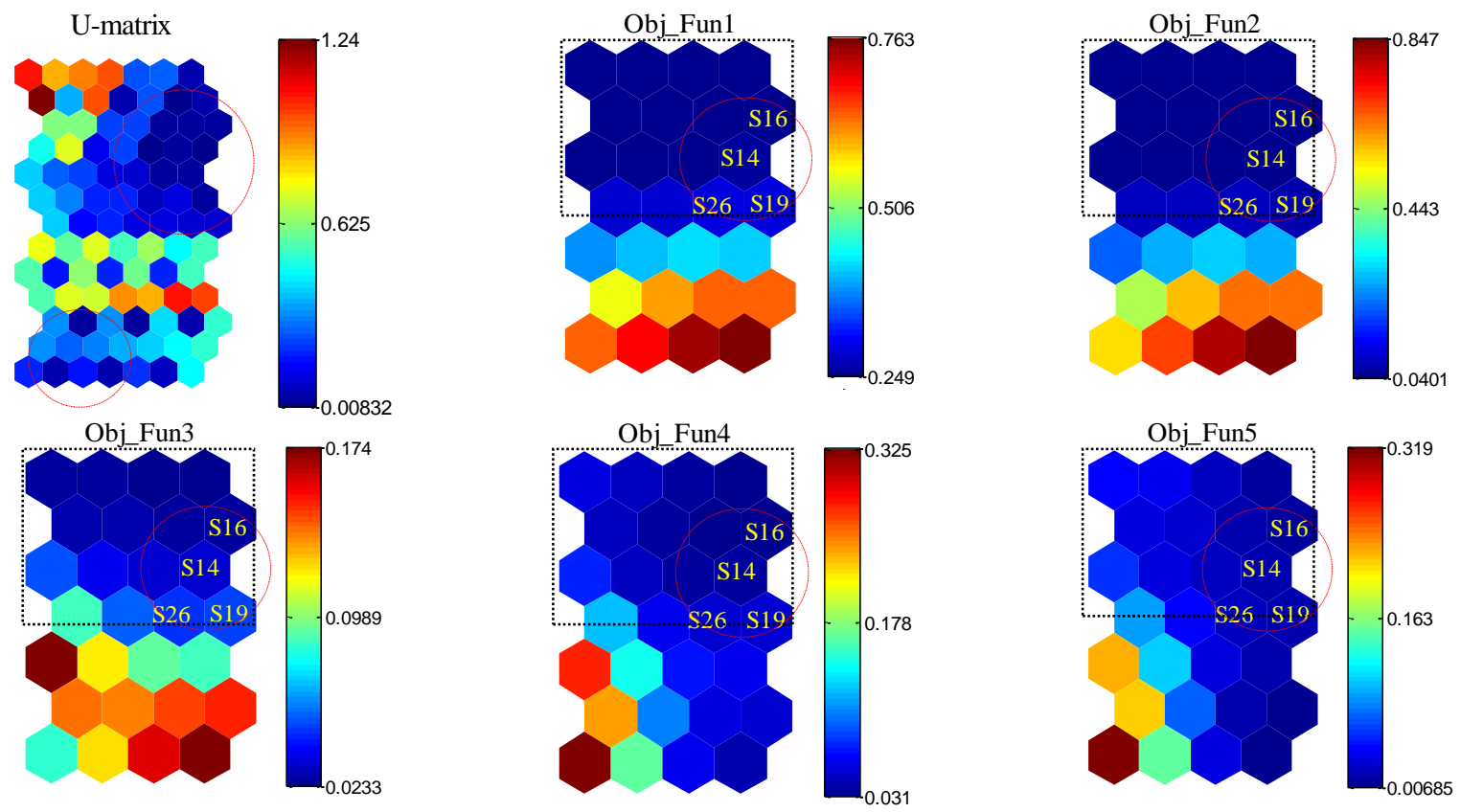

Fig. 19: SOM: the U-matrix and the five objective functions 


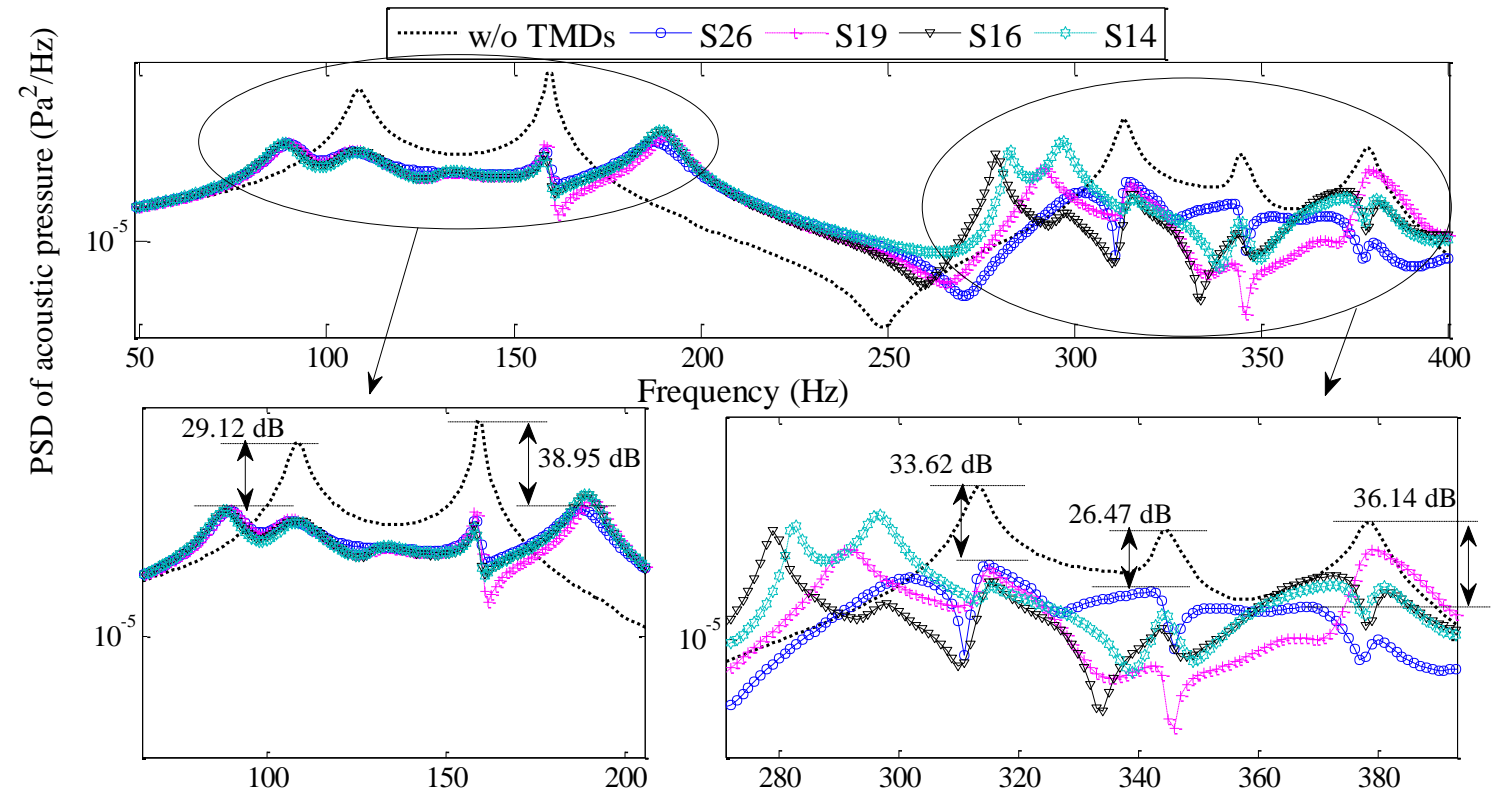

Fig. 20: PSD responses for four optimal solutions (sound control using five TMDs) 
Supplementary Material
Click here to download Supplementary Material: summary of what is different_MSSP.docx

Supplementary Material
Click here to download Supplementary Material: summary of what is different_MSSP.docx

\begin{abstract}
Ch here to download Supplementary Material: summary of what is different MSSP.docx
\end{abstract}

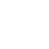

